

\section{DISCLAIMER}

This report was prepared as an account of work sponsored by an agency of the United States Government. Neither the United States Government nor any agency Thereof, nor any of their employees, makes any warranty, express or implied, or assumes any legal liability or responsibility for the accuracy, completeness, or usefulness of any information, apparatus, product, or process disclosed, or represents that its use would not infringe privately owned rights. Reference herein to any specific commercial product, process, or service by trade name, trademark, manufacturer, or otherwise does not necessarily constitute or imply its endorsement, recommendation, or favoring by the United States Government or any agency thereof. The views and opinions of authors expressed herein do not necessarily state or reflect those of the United States Government or any agency thereof. 


\section{DISCLAIMER}

Portions of this document may be illegible in electronic image products. Images are produced from the best available original document. 
Issued by Sandia National Laboratories, operated for the United States Department of Energy by Sandia Corporation.

NOTICE: This report was prepared as an account of work sponsored by an agency of the United States Govermment. Neither the United States Government nor any agency thereof. nor any of their employees, nor any of their contractors, subcontractors, or their employees, makes any warranty, express or implied, or assumes any legal liability or responsibility for the accuracy, completeness, or usefuness of any information, apparatus, product, or process disclosed, or represents that its use would not infringe privately owned rights. Reference herein to any specific commercial product, process, or service by trade name, trademark, manufacturer, or otherwise, does not necessarily constitute or mply its endorsement, recommendation, or favoring by the United States Government. any agency thereof or any of their contractors or subcontractors. The views and opinions expressed herein do not necessarily state or reflect those of the United States Government, any agency thereof or any of their contractors or subcontractors.

Printed in the United States of America Available from

National Technical Information Service

U.S. Department of Commerce

5285 Port Royal Road

Springfield, VA 22161

NTIS price codes

Printed copy: $\$ 7.00$

Microfiche copy: $A 01$ 


\title{
Prediction of Forces and Moments on Finned Bodies at High Angle of Attack in Transonic Flow
}

William L. Oberkampf*

Aeroballistics Division 5631

Sandia National Laboratories

Albuquerque, NM 87185

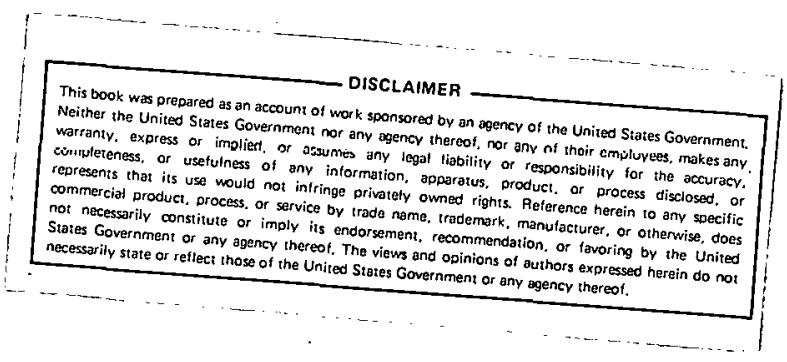

\begin{abstract}
This report describes a theoretical method for the prediction of fin forces and moments on bodies at high angle of attack in subsonic and transonic flow. The body is assumed to be a circular cylinder with cruciform fins (or wings) of arbitrary planform. The body can have an arbitrary roll (or bank) angle, and each fin can have individual control deflection. The method combines a body vortex flow model and lifting surface theory to predict the normal force distribution over each fin surface. Extensive comparisons are made between theory and experiment for various planform fins. A description of the use of the computer program that implements the method is given.
\end{abstract}

*This work was sponsored in part by the Air Force Armament Laboratory (AFATL/DLJ), Eglin AFB, FL, under Air Force Contract No. F08635-77-C-0049. The contract was in support of research done while the author was on the faculty of the Department of Mechanical Engineering, University of Texas at Austin. The contract monitor for AFATL was D. C. Daniel (DLJ). 


\section{Acknowledgment}

The author thanks D. C. Daniel, Aerodynamics Research Manager of the Air Force Armament Laboratory, Eglin AFB, FL for his comments and suggestions during the investigation. 


\section{Contents}

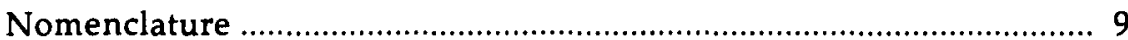

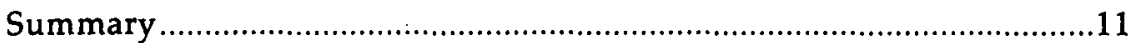

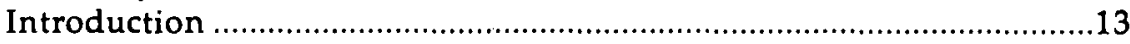

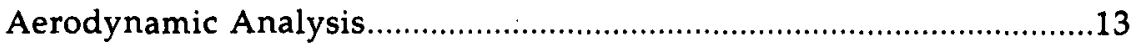

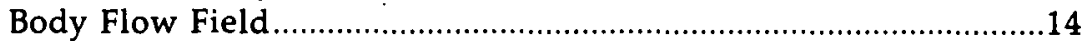

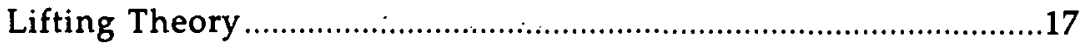

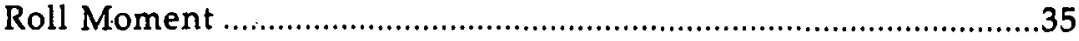

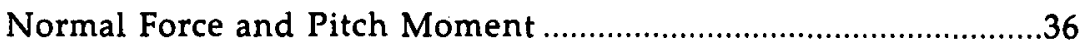

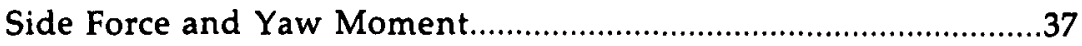

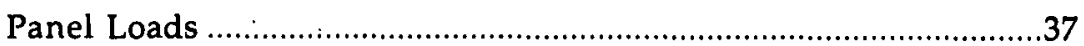

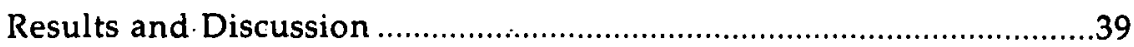

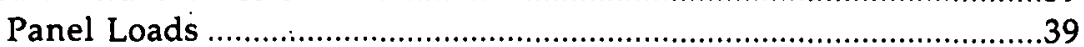

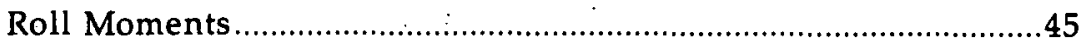

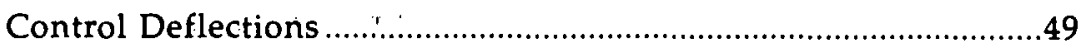

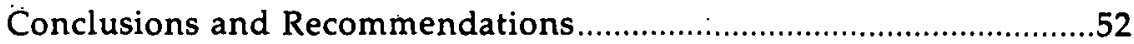

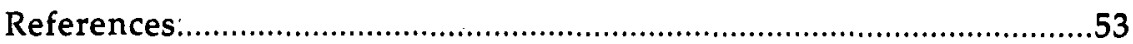

APPENDIX A--Structure and Listing of Computer Program ...............55

APPENDIX B--Use of the Computer Program.......................................71

\section{Illustrations}

\section{Figure}

1 Coordinate System and Schematic of Body Vortices .................. 15

2 Cross-Flow Plane Flow Model ................................................. 16

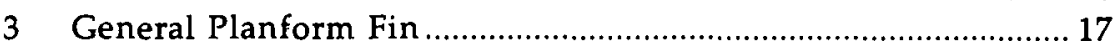

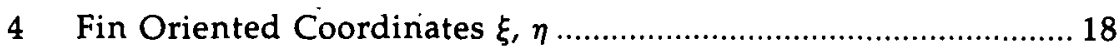

5 . Normal Force Distribution Over Fin Surface

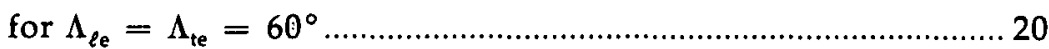

6 Normal Force Distribution Over Fin Surface

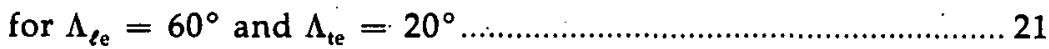

7. Normal Force Distribution Over Fin Surface for $\Lambda_{l \mathrm{e}}=\Lambda_{\mathrm{te}}=0^{\circ}$

8 Normal Force Distribution Over Fin Surface

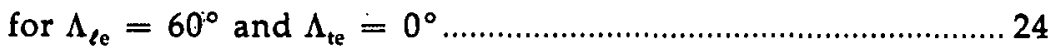

9 Normal Force Distribution Over Fin Surface

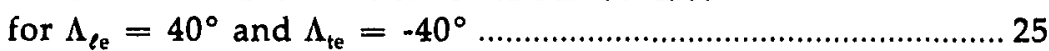

10 Surface Nurmal Vector and Sign Convention for Fin Control Deflection .......................................................... 26

1:1 Fin Surface Cylindrical Coordinates ........................................ 27

12 Geometry for Determining Effective Aspect Ratio ...................... 28

13 Normal Force Coefficient vs $\alpha$ for Rectangular

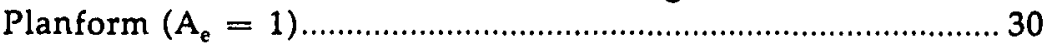

14 Normal Force Coefficient vs $\alpha$ for Diamond

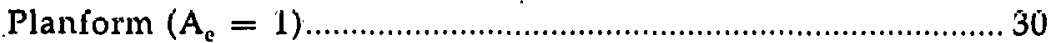

15 Normal Force Coefficient vs $\alpha$ for Clipped Delta

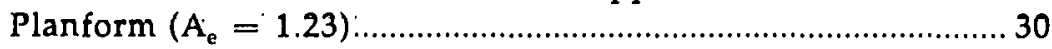

16 Normal Force Coefficient vs $\alpha$ for Delta

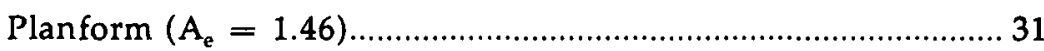

17 Normal Force Coefficient vs $\alpha$ for Rectangular

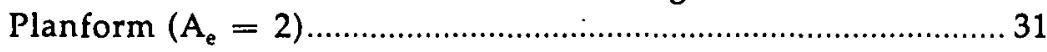

18 Normal Force Coefficient vs $\alpha$ for Arrow Planform $\left(\Lambda_{e}-2\right)$ 


\section{Illustrations (Cont)}

19 Normal Force Coefficient vs $\alpha$ for Delta

Planforin $\left(A_{e}=2.31\right)$.

20 Normal Force Coefficient vs $\alpha$ for Clipped Arrow

Planform $\left(A_{e}=2.61\right)$.

21 Normal Force Coefficient vs $\alpha$ for Trapezoidal

Planform $\left(A_{e}=3\right.$.).

22 Normal Force Coefficient vs $\alpha$ for Delta

Planform $\left(\mathrm{A}_{\mathrm{e}}=4\right.$.)...

23 Control Interference for Pitch and Roll Control

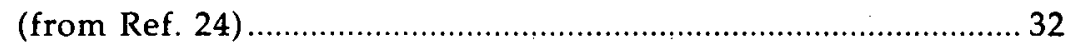

24 Control Interference for Individual Panel Deflection ................ 33

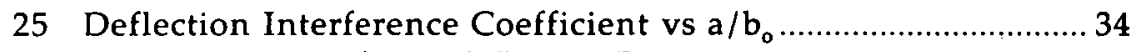

26 Induced Angle of Attack Due to Rolling Speed ........................... 34

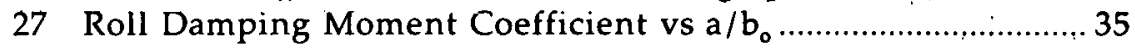

28 Components of the Fin Normal Force ......................................... 37

29 Sign Convention for Panel Normal Force, Hinge Moment, and Root Bending Moment ......................................................... 38

30 Fin Planforms Used for Comparison of Theory and Experiment ......................................................................... 40

31 Windward Panel Normal Force vs Roll Angle for

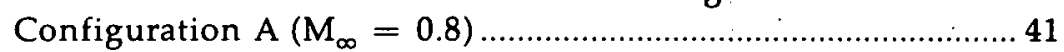

32 Windward Panel Normal Force vs Roll Angle for Configuration $\mathrm{A}\left(\mathrm{M}_{\infty}=1.22\right)$.

33 Leeward Panel Normal Force vs Roll Angle for Configuration $\mathrm{A}\left(\mathrm{M}_{\infty}=0.8\right)$

34 Leeward Panel Normal Force vs Roll Angle for Configuration $\mathrm{A}\left(\mathrm{M}_{\infty}=1.22\right)$.

35 Windward Fin Root Bending Moment vs Roll Angle for Contiguration $\mathrm{A}\left(\mathrm{M}_{\infty}=U .8\right)$.

36 Windward Fin Root Bending Moment vs Roll Angle for Configuration $\mathrm{A}\left(\mathrm{M}_{\infty}=1.22\right)$.

37 Leeward Fin Root Bending Moment vs Roll Angle for Contiguration $\mathrm{A}\left(\mathrm{M}_{\infty}=\mathrm{U} .8\right)$.

38 Leeward Fin Root Bending Moment vs Roll Angle for Configuration $A\left(M_{\infty}=1.22\right)$.

39 Windward Fin Hinge Moment vs Roll Angle for Configuration $\mathrm{A}\left(\mathrm{M}_{\infty}=0.8\right)$

40 Windward Fin Hinge Moment vs Roll Angle for Configuration $\mathrm{A}\left(\mathrm{M}_{\infty}=1.22\right)$.

41 Leeward Fin Hinge Moment vs Roll Angle for

Cunfigul'aliun $\mathrm{A}\left(\mathrm{M}_{\infty}=0.8\right)$

42 Leeward Fin Hinge Moment vs Roll Angle for Configuration $A\left(M_{\infty}=1.22\right)$.

43 Induced Roll Moment vs $\alpha_{\mathrm{b}}$ for Configuration A $\left(\mathrm{M}_{\infty}=0.8\right)$.

44 Induced Roll Moment vs $\alpha_{\mathrm{b}}$ for Configuration A $\left(\mathrm{M}_{\infty}=1.22\right)$. . .46

45 Spanwise Fin Loading for $\alpha_{\mathrm{b}}=20^{\circ}, \phi=20^{\circ}$ Configuration A $\left(\mathrm{M}_{\infty}=0.8\right)$

46 Induced Roll Moment vs $\alpha_{\mathrm{b}}$ for Configuration B........................ 46

47 Induced Roll Moment vs $\alpha_{\mathrm{b}}$ for Configuration D...................... 47

48 Roll Damping Moment vs $\alpha_{b}$ for Configuration E....................... 47 


\section{Illustrations (Cont)}

49 Roll Damping Moment vs $\alpha_{b}$ for Configuration F...................... 48

50 Steady-State Roll Rate vs $\alpha_{\mathrm{b}}$ for Configuration E

( $4^{\circ}$ fin cant)

51 Interference Lift Ratio vs $a / b_{0}$ (from Ref. 24).

52 Pitch Control Force vs $\alpha_{\mathrm{b}}$ for Configuration B

$\left(\phi=0^{\circ}, \delta_{1}=\delta_{3}=10^{\circ}, \delta_{2}=\delta_{4}=0^{\circ}\right)$.

53 Pitch Control Force vs $\alpha_{\mathrm{b}}$ for Configuration C $\left(\phi=0^{\circ}, \delta_{1}=\delta_{3}=10^{\circ}, \delta_{2}=\delta_{4}=0^{\circ}\right)$

54 Pitch Control Force vs $\alpha_{\mathrm{b}}$ for Configuration D

$\left(\phi=0^{\circ}, \delta_{1}=\delta_{3}=10^{\circ}, \delta_{2}=\delta_{4}=0^{\circ}\right)$

55 Pitch Control Force vs $\alpha_{\mathrm{b}}$ for Configuration B

$\left(\phi=45^{\circ}, \delta_{1}=\delta_{2}=\delta_{3}=\delta_{4}=10^{\circ}\right)$

56 Pitch Control Force vs $\alpha_{\mathrm{b}}$ for Configuration C $\left(\phi=45^{\circ}, \delta_{1}=\delta_{2}=\delta_{3}=\delta_{4}=10^{\circ}\right)$

57 Pitch Control Force vs $\alpha_{\mathrm{b}}$ for Configuration D $\left(\phi=45^{\circ}, \delta_{1}=\delta_{2}=\delta_{3}=\delta_{4}=10^{\circ}\right)$

58 Roll Control Moment vs $\alpha_{\mathrm{b}}$ for Configuration B $\left(\phi=0^{\circ}, \delta_{1}=-\delta_{3}=10^{\circ}, \delta_{2}=\delta_{4}=0^{\circ}\right)$

59 Roll Control Moment vs $\alpha_{\mathrm{b}}$ for Configuration C $\left(\phi=0^{\circ}, \delta_{1}=-\delta_{3}=10^{\circ}, \delta_{2}=\delta_{4}=0^{\circ}\right)$

60 Roll Control Moment vs $\alpha_{\mathrm{b}}$ for Configuration D $\left(\phi=0^{\circ}, \delta_{1}=-\delta_{3}=10^{\circ}, \delta_{2}=\delta_{4}=0^{\circ}\right)$ 


\section{Nomenclature}

a Body radius

$A_{e} \quad$ Aspect ratio of fins based on exposed planform area, $\mathrm{Eq}(19 \mathrm{c})$

$A_{p} \quad$ Planform aspect ratio of fins based on extension through the body $b^{2} / S_{f_{p}}$

b Fin span

b. Fin semispan

B Root bending moment (Figure 29)

$\mathrm{C}_{\ell} \quad$ Roll moment coefficient $\left(\ell / \mathrm{q}_{\infty} \mathrm{S}_{\mathrm{b}} \mathrm{d}\right)$

$\mathrm{C}_{\ell \mathrm{p}} \quad$ Roll damping moment coefficient derivative $\left(\partial \mathrm{C}_{\ell} / \partial \mathrm{p}\right)_{\mathrm{p} \rightarrow \mathrm{o}}$

$\mathrm{C}_{m} \quad$ Pitch moment coefficient $\left(m / \mathrm{q}_{\infty} \mathrm{S}_{\mathrm{b}} \mathrm{d}\right)$

$\mathrm{C}_{n} \quad$ Yaw moment coefficient $\left(n / \mathrm{q}_{\infty} \mathrm{S}_{\mathrm{b}} \mathrm{d}\right)$

$\mathrm{C}_{\mathrm{n}} \quad$ Local normal force coefficient of fin, Eq (14)

$C_{N} \quad$ Local normal force coefficient of fin due to angle of attack, Eq (33)

$\mathbf{C}_{\mathbf{N}_{\alpha}} \quad$ Normal force coefficient derivative of fin $\left(\partial \mathrm{C}_{N} / \partial \alpha\right)_{\alpha \rightarrow 0}$

$C_{b} \quad$ Panel root bending moment coefficient $\left[B / q_{\infty}\left(b_{o}-a\right) S_{f}\right]$

$C_{h} \quad$ Panel hinge moment coefficient $\left(H / q_{\infty} c_{r} S_{f}\right)$

$C_{p} \quad$ Panel normal force coefficient $\left(N / q_{\infty} S_{f}\right)$

$c_{x} \quad$ Root chord of fin

$C_{y} \quad$ Side force coefficient $\left(F_{y} / q_{\infty} S_{b}\right)$

$C_{z} \quad$ Normal force coefficient $\left(\mathrm{F}_{z} / \mathrm{q}_{\infty} \mathrm{S}_{\mathrm{b}}\right)$

d Body diameter

$F_{x}, F_{y}$ Forces in the $x, y, z$ directions, respectively

$\mathrm{F}_{2} \quad$ (Figure 1)

H Panel hinge moment (Figure 29)

$\vec{i}, \vec{j}, \vec{k} \quad$ Unit vectors in the $x, y, z$ directions, respectively

$\mathbf{I}_{\mathbf{d}} \quad$ Fin deflection interference coefficient,

Eq (39)

I Rolling-motion interference coefficient,

Eq (44)

$\mathbf{k}_{\mathbf{B}} \quad$ Interference lift ratio (Figure 51)

$K_{p} \quad$ Jotential flow lift coefficient, Eq (34h)

$K_{v} \quad$ Vortex flow lift coefficient, Eq (34i)

$L_{s} \quad$ Length of the vortex sheet, Eq (6a)

$\ell, m, n \quad$ Roll, pitch, and yaw moments, respectively (Figure 1)

M Mach number

$\vec{n} \quad$ Unit normal vector for fin surface
N Normal force

$\mathbf{N}_{\mathbf{v}} \quad$ Total number of vortices in cross-flow plane

p Dimensionless roll speed $\left(\dot{\phi} \mathrm{b}_{0} / \mathrm{U}_{\infty}\right)$

q : Dynamic pressure $\left(1 / 2 \rho \mathrm{U}^{2}\right)$

$\mathbf{r}_{\text {c }} \quad$ Radius of vortex core

$\mathbf{r}, \phi \quad$ Polar coordinates in cross-flow plane

$\mathbf{r}_{1}, \phi_{1} \quad$ Coordinates of primary body vortex

Complex position in the cross-flow plane $(\mathrm{y}+\mathrm{iz})$

$S_{b} \quad$ Frontal area of missile body $\left(\pi a^{2}\right)$

$S_{f} \quad$ Exposed planform area of fin, Eq (19d)

$\mathbf{S}_{f_{p}} \quad$ Planform area of two fins including extension through the body, Eq (40b)

$u, v, w$ Velocities in the $x, y, z$ directions, respectively (Figure 1)

U Magnitude of velocity

$U_{c} \quad$ Freestream cross-flow velocity, $U_{\infty} \sin \alpha_{b}$

$\mathbf{v}_{\mathbf{c}}, \mathbf{w}_{\mathbf{c}} \quad$ Cross-flow velocities in the $\mathrm{y}, \mathrm{z}$ directions, respectively, Eq (2)

$\vec{v} \quad$ Flow field velocity $(u \vec{i}+v \vec{j}+w \vec{k})$

$\mathbf{x}, \mathbf{y}, \mathbf{z} \quad$ Body Cartesian coordinates (Figure 1)

$\mathbf{y}_{v}, \mathbf{z}_{\mathbf{v}} \quad$ Location of primary body vortex in crossflow plane

$\alpha \quad$ Angle of attack

$\alpha_{\ell} \quad$ Local angle of attack

$\alpha_{s} \quad$ Stall angle of attack of the fin, Eq (35b)

$\Gamma_{p} \quad$ Strength of the primary body vortex

$\Gamma_{s} \quad$ Stength of vortex sheet

$\delta_{j} \quad$ Control deflection of the $j$ 'th fin (Figure 10)

$\sigma \quad$ Geometric scaling factor for fin, $\mathrm{Eq} \mathrm{(20a)}$

$\Lambda \quad$ Sweep angle of fin

$\xi, \eta \quad$ Fin-oriented surface coordinates, Eq (18)

$\rho \quad$ Fluid density

$\phi \quad$ Roll angle (Figure 10)

$\dot{\phi} \quad$ Roll rate $(\mathrm{rad} / \mathrm{s})$

\section{Subscripts:}

b . Missile body

e "Effective" value of argument

le Leading edge

te Trailing edge

$\infty \quad$ Freestream conditions

\section{Superscripts:}

- Derivative with respect to time

A "Effective" value of argument 


\section{Summary}

The present investigation is concerned with the theoretical prediction of fin forces and moments on bodies at high angle of attack in subsonic and transonic flow. The body is assumed to be a circular cylinder with a streamlined nose and only cruciform fins (or wings) as attached lifting surfaces. The fins are assumed to be planar and have straight leading, trailing and tip chord edges. The leading edge can have arbitrary sweep back and the trailing edge can be swept back or forward. The body can have an arbitrary roll (or bank) angle and each fin can have arbitrary control deflection. The vortices shed from the body are assumed to be symmetrically located with respect to the angle of attack plane and of equal strength but opposite rotational sense. The highest angle of attack of the body for which the body vortices remain symmetric depends on the nose fineness ratio, body fineness ratio, and Mach number; normally this angle is near $25^{\circ}$. The body flow field model and lifting theory use some empirical data, but the user of the method need not provide any additional data. A description of the construction and use of the program FINLOAD, which implements the method, is given.

Extensive comparisons are made between predicted results and experimental measurements. Included in the comparisons are: panel normal force, root bending moment, induced roll moment, nonlinear roll damping moment, pitch (or yaw) control forces, and roll control moment. The force and moment predictions are compared with experimental data for six different fin geometies; these include delta, clipped delta, and rectangular planforms. Predictions for wing alone normal force characteristics are compared with data for rectangular, delta, clipped delta, diamond, arrow, clipped arrow, and trapezoidal planforms. Extensive discussions are given that explain the underlying aerodynamic causes of fin force and moment nonlinearities and how these are related to fin geometry. Good agreement between the present method and experiment is obtained, except for control force and moment where the agreement could be characterized as "fair." 


\section{Prediction of Forces and Moments on Finned Bodies at High Angle of Attack in Transonic Flow}

\section{Introduction}

The aerodynamics of missiles and bombs at high angle of attack has become increasingly important for modern design requirements. Examples of this are high maneuverability of air-to-air-missiles and tolerance of large disturbances at store separation. At high angle of attack, a body of revolution sheds two symmetric vortices from the leeside of the body, and these grow in strength along the length of the body. The body's attached lifting surfaces are immersed in this vortex wake flow and, consequently, the surface pressure distributions are significantly changed from the potential flow case. This, in turn, causes nonlinearities in the forces and moments produced by the lifting surfaces. These nonlinearities have been known to cause serious flight stability and controllability problems in vehicle flight dynamics.

Attempts at predicting the forces and moments produced by lifting surfaces in a symmetric bodyvortex wake have met with moderate success. Very early work was done by Mello and Sivier ${ }^{1}$ for a cruciform-fin missile in supersonic flow. References 2 and 3 report reasonably successful work for incompressible and supersonic flow, respectively, but only rectangular fin planforms were considered. The approach taken was to calculate the. body-flow field using a vortex-modeling technique and then use this as input to a lifting theory. 2,3 The most extensive work on the subject has been achieved by Nielson and his associates.47 They have attacked the very difficult problem of a general canard-fin-body configuration in transonic and supersonic flow with both symmetric and asymmetic body vortices and canard vortices. Their approach is a combination of slender-body theory, Deffenbaugh's $s^{8.9}$ method for the impulse flow analogy, and data base experimental input for fin (or wing) alone characteristics.

The present approach uses the body flow field model developed in Reference 10 and combines it with an approximate lifting surface theory to predict fin forces and moments. The lifting surface theory and the equations for fin forces and moments are developed in detail. The applicability and accuracy of the present method is evaluated by extensive comparisons of theory and experiment. The purpose of the present work is to develop a force and moment predictive method that is applicable to a wide variety of fin planforms over the subsonic and transonic speed range.

\section{Aerodynamic Analysis}

The general approach to the aerodynamics of the problem is to. calculate the body flow field and then calculate the forces and moments of attached lifting surfaces exposed to this flow field. This approach is clearly based on the assumption that the body flow field is not significantly affected by the flow induced by lifting surfaces. This assumption implies that the present analysis is not appropriate for missile configurations in which the fin-root chord is a large portion of the length of the missile body (e.g., the Phoenix air-to-air missile (AIM-54A)). The present analysis also assumes that there is only one set of lifting surfaces (wings or fins) and that it is arranged in a cruciform configuration. The present approach could be applied to a two- or three-fin configuration by making appropriate modifications to the lifting theory.

This present approach naturally divides the analysis into two areas: the body flow field and the prediction of lifting surface forces and moments. The model of the body flow field was developed previously in References 2 and 10. For completeness, however, the model and the associated computational procedures are described in this report. The prediction of lifting surface forces and moments is described in two phases. First, the lifting theory for calculating the normal force distribution and the 
total normal force of the lifting surface in uniform approach flow is described. Second, the model of the body flow field and the lifting theory are combined to yield a method for predicting forces and moments of attached lifting surfaces.

\section{Body Flow Field}

The flow field of a circular cylindrical body at high angle of attack is dominated by the presence of body vortices and their associated feeding sheets. Figure 1 shows the coordinate system and a schematic of the body vortex wake flow. These vortices increase in strength as the angle of attack or body length increases. To model this complex separated flow, the flow is divided into the cross-flow components ( $v_{c}$ and $w_{c}$ ) and the axial flow component, $U_{\infty} \cos \alpha_{b}$. The local flow velocity can then be expressed as:

$$
\bar{V}=U_{\infty} \cos \alpha_{b} \bar{i}+v \bar{j}+w_{c} \bar{k} .
$$

It is assumed that the steady, three-dimensional, body flow field can be divided into (1) constant axialflow component and (2) the two-dimensional, potential, flow about a circular cylinder with vortices in the wake and their associated image vortices inside the cylinder. Essentially all of the vorticity is located inside the vortex cores of the primary body vortices and the vortex feeding sheets connecting the body boundary layer separation points and the primary body vortices. Figure 2 shows the primary and sheet vortices in the cross-flow plane. Using this model, the cross-flow velocity components can be written as

$$
\begin{aligned}
v_{c}= & {\left[\frac{-2 U_{c} a^{2} y z}{\left(y^{2}+z^{2}\right)^{2}}+\frac{\Gamma_{p}}{2 \pi} \sum_{j=1}^{4}(-1)^{j} \frac{z-z_{j}}{\left(y-y_{j}\right)^{2}+\left(z-z_{j}\right)^{2}}\right.} \\
& \left.+\frac{2 \Gamma_{s}}{\pi\left(N_{v}-4\right)} \sum_{j=5}^{N_{v}}(-1)^{i} \frac{z-z_{j}}{\left(y-y_{j}\right)^{2}+\left(z-z_{j}\right)^{2}}\right]\left[1-e^{-C}\right]
\end{aligned}
$$

$$
\begin{aligned}
w_{c}= & \left\{U_{c}\left[1+\frac{a^{2}\left(y^{2}-z^{2}\right)}{\left(y^{2}+z^{2}\right)^{2}}\right]-\frac{\Gamma_{p}}{2 \pi} \sum_{j=1}^{4}(-1)^{j} .\right. \\
& \frac{y-y_{j}}{\left(y-y_{j}\right)^{2}+\left(z-z_{j}\right)^{2}}-\frac{2 \Gamma_{s}}{\pi\left(N_{v}-4\right)} \sum_{j=5}^{N_{v}}(-1)^{j} \\
& \left.\frac{y-y_{j}}{\left(y-y_{j}\right)^{2}+\left(z-z_{j}\right)^{2}}\right\}\left\{1-e^{-c}\right\}
\end{aligned}
$$

where

$$
\begin{aligned}
& C=1.254\left[\left(y-y_{v}\right)^{2}+\left(z-z_{v}\right)^{2}\right] / r_{c}{ }^{2} \\
& y_{v}, z_{v}= \begin{cases}y_{1}, z_{1} & y \geq 0 \\
y_{4}, z_{4} & y<0\end{cases} \\
& \mathrm{U}_{\mathrm{c}}=\mathrm{U}_{\infty} \sin \dot{\alpha}_{\mathrm{b}} \text { is the free-stream cross-flow } \\
& \text { velocity } \\
& \text { a . = body radius } \\
& \Gamma_{\mathrm{p}} \quad=\text { strength of a primary body vortex } \\
& \Gamma_{\mathrm{s}} \quad=\text { strength of a vortex sheet } \\
& y_{j}, z_{j}=\text { Incation of the } j^{\prime} \text { th vortex } \\
& \mathrm{N}_{\mathrm{v}}=\text { total number of vortices in the cross-flow } \\
& \text { plane. } \\
& \mathbf{r}_{\mathrm{c}} \quad=\text { vortex core radius }
\end{aligned}
$$

The depentence of $\Gamma_{p}, \Gamma_{s}, y_{1}, z_{1}$, and $i_{c}$ wis astyle of attack and body length is taken from experimental measurements.

The expnnential-term in Fqs (2a) and (2b) mas included to model the solid body type rotation in the cores of the primary body vortices. This method of approximating the vortex cores yields a cross-flow velocity field that is continuous, whereas simply imposing a solid body rotation onto a potential flow field does not.

Using the assumption of ą symmetric vortex wake, the relationship between the locations of all of the vortices in the cross-flow plane becomes

$\left.\begin{array}{l}y_{1}=-y_{1+1}=a^{2} y_{j-1} /\left(y_{j-1}^{2}+z_{j-1}^{2}\right) \\ z_{j}=z_{j+1}=a^{2} z_{j-1} /\left(y_{j-1}^{2}+z_{j-1}^{2}\right)\end{array}\right\}$ for $j=2,6,10, \ldots N_{v}-2$

$y_{j}^{\prime}=-y_{j-3}$ and $z_{i}=z_{j-3} \quad$ for $j=4,8,12, \ldots N_{v}$.

With these equations the location of all of the vortices can be related to the location of the vortices external to the body in the positive $(y, z)$ quadrant; i.e., $j=$ $1,5,9, \ldots \mathrm{N}_{\mathrm{v}}-3$.

The location of the right-handed vortex sheet, $s_{s}$ $=r_{s} e^{i \phi}$, is given as

$$
\begin{aligned}
s_{s}= & {\left[a \cos \left(\pi \phi / 2 \phi_{1}\right)+\left(r_{1}+r_{c}\right) \sin ^{2}\left(\pi \phi / 2 \phi_{1}\right) /\right.} \\
& \left.\left\{1+\left(r_{1}+r_{c}\right)\left(\phi_{1}-\phi\right) / a\right\}\right] \mathrm{e}^{\mathrm{i} \phi} \quad \phi_{s s} \leq \phi \leq \phi_{1}
\end{aligned}
$$


where $r_{1}$ and $\phi_{1}$ are the radial and angular locations, respectively, of primary vortex number 1 , and $\phi_{\mathrm{ss}}$ is the angle at which the sheet separates from the body. The vortex sheet location as given by Eq (4) yields a slight improvement in comparison with experimental data of Reference 11 as compared with that used in References 2 and 10. Equation (4) requires that the vortex sheet terminates at the core radius of the primary vortex, whereas the equations used in References 2 and 10 terminated the sheet at the center of the primary vortex.

$\phi_{s s}$ is defined as the angle at which the radial location of the sheet achieves a value of 1.01a; therefore, $\phi_{\mathrm{ss}}$ is defined by the equation

$$
\begin{gathered}
\cos \left(\pi \phi_{s s} / 2 \phi_{1}\right)+"\left(r_{1}+r_{c}\right) \sin ^{2}\left(\pi \phi_{s s} / 2 \phi_{1}\right) / \\
{\left[a+\left(r_{1}+r_{c}\right)\left(\phi_{1}-\phi_{s s}\right)\right]=1.01}
\end{gathered}
$$

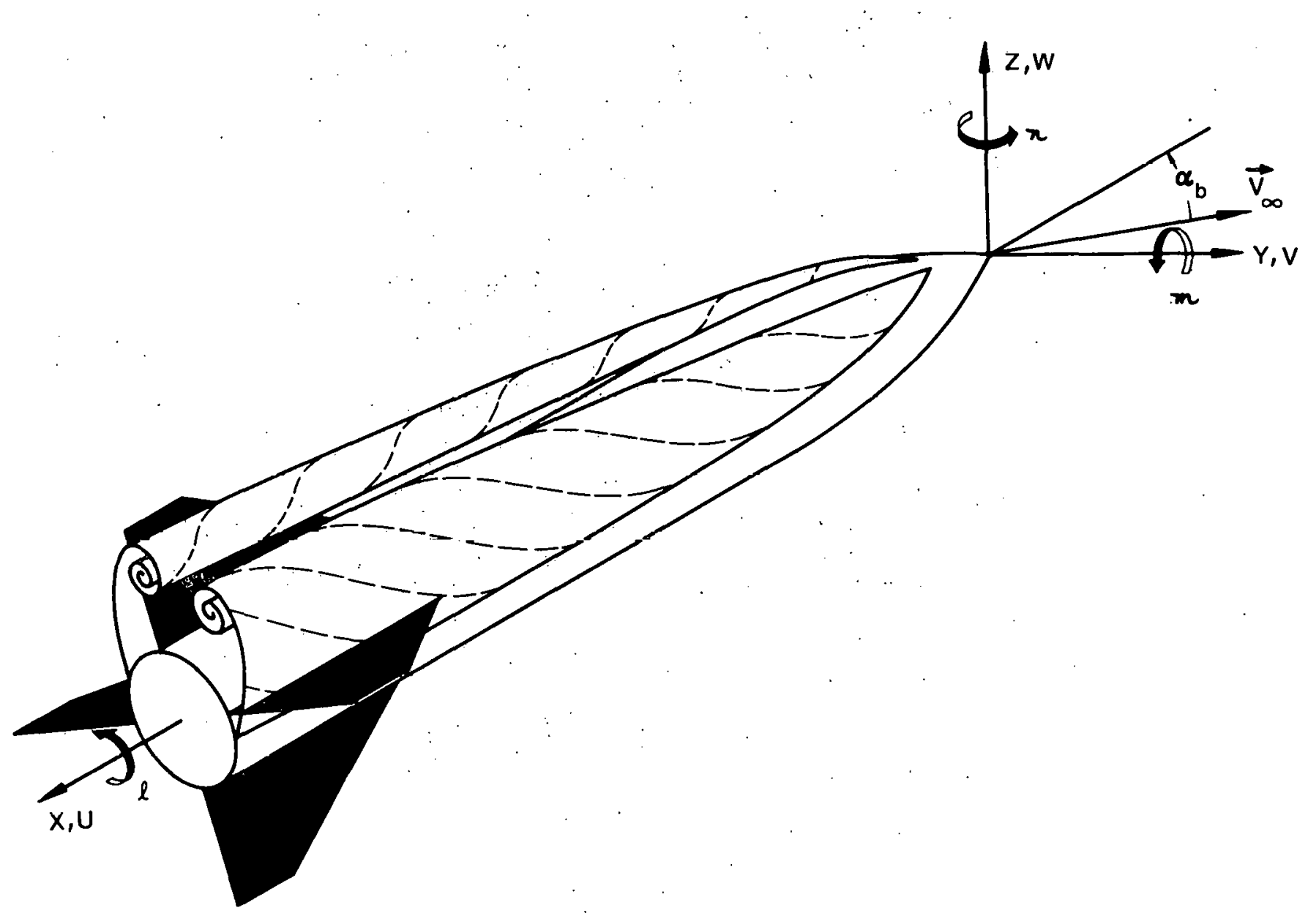

The low-strength vortices which represent the vortex sheet are equally spaced in the arc length along the sheet. The arc length of the vortex sheet is

where $r_{s}$ is the magnitude of $s_{s}$, from Eq (4), and

$$
\mathrm{L}_{\mathrm{s}}=\int_{\phi_{\mathrm{ss}}}^{\phi_{1}}\left[\mathrm{r}_{\mathrm{s}}^{2}+\left(\frac{\mathrm{dr} \mathrm{r}_{\mathrm{s}}}{\mathrm{d} \phi}\right)^{2}\right]^{1 / 2} \mathrm{~d} \phi
$$

$$
\begin{aligned}
\frac{\mathrm{dr} r_{s}}{\mathrm{~d} \phi}= & -\left(\mathrm{a} \pi / 2 \phi_{1}\right) \sin \left(\pi \phi / 2 \phi_{1}\right)+\left[\left\{\left(\mathrm{r}_{1}+\mathrm{r}_{\mathrm{c}}\right) \pi / \phi_{1}\right\}\right. \\
& \sin \left(\pi \phi / 2 \phi_{1}\right) \cos \left(\pi \phi / 2 \phi_{1}\right)\left\{1+\left(\mathrm{r}_{1}+\mathrm{r}_{\mathrm{c}}\right) .\right. \\
& \left.\left.\left(\phi_{1}-\phi\right) / \mathrm{a}\right\}+\left\{\left(\mathrm{r}_{1}+\mathrm{r}_{\mathrm{c}}\right)^{2} / \mathrm{a}\right\} \sin ^{2}\left(\pi \phi / 2 \phi_{1}\right)\right] / \\
& \left\{1+\left(\mathrm{r}_{1}+\mathrm{r}_{\mathrm{c}}\right)\left(\phi_{1}-\phi\right) / \mathrm{a}\right\}^{2} .
\end{aligned}
$$

Figure 1. Coordinate System and Schematic of Body Vortices 


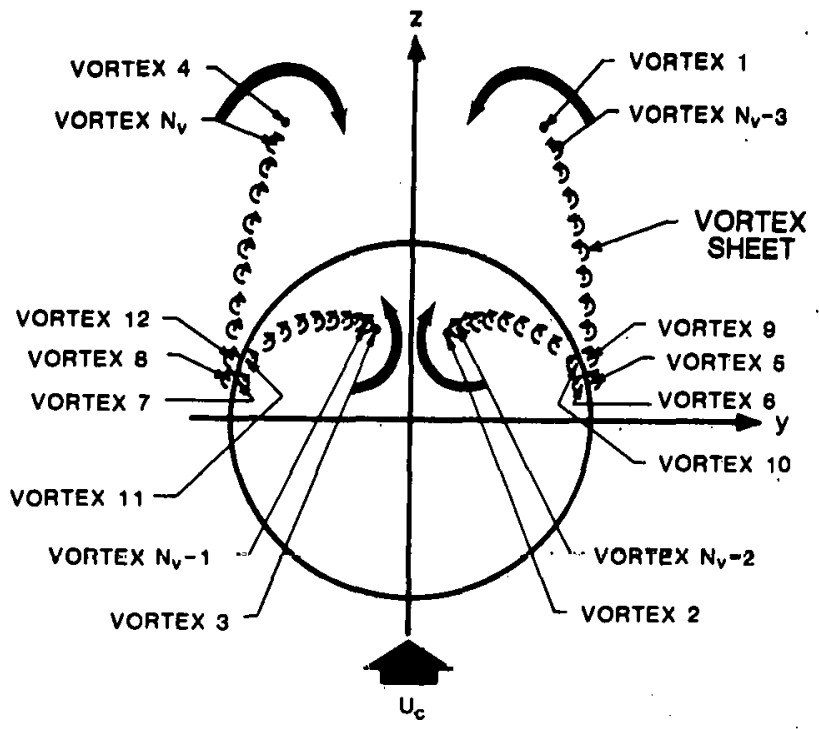

Figure 2. Cross-Flow Plane Flow Model

The first vortex in the sheet (vortex 5) is located at the point where the sheet leaves the body so that $\mathrm{r}_{5}=1.01 \mathrm{a}$ and $\phi_{5}=\phi_{\mathrm{ss}}$. The angular position for vortices $5,9,13, \ldots \mathrm{N}_{\mathrm{v}}-3$ is found from

$$
\left(\frac{j-5}{N_{v}-4}\right) L_{s}=\int_{\phi_{s s}}^{\phi_{i}}\left[r_{s}^{2}+\left(\frac{d r_{s}}{d \phi}\right)^{2}\right]^{1 / 2} d \phi .
$$

Once the angular position is known from $\mathrm{Eq}(7)$, then from $\mathrm{Eq}(4)$ one obtains the radial location:

$$
\begin{aligned}
r_{j}= & a \cos \left(\pi \phi_{j} / 2 \phi_{1}\right)+\left(r_{1}+r_{c}\right) \sin ^{2}\left(\pi \phi_{j} / 2 \phi_{1}\right) / \\
& \left\{1+\left(\dot{r}_{1}+r_{c}\right)\left(\phi_{1}-\phi_{j}\right) / a\right\}
\end{aligned}
$$

The experimental inputs required by the theory will now be discussed. The total strength of all the vorticity, $\Gamma_{t}$ (primary vortex and feeding sheet), in each half-plane of the wake is taken from the experimental data of Grosrhe ${ }^{12}$

$\Gamma_{1} /\left(\pi d U_{\infty}\right)=0.35(x / a-6) \alpha_{b}^{2}$ for $x / a>6$.

This equation represents the data of Grosche for $7^{\circ} \leq \alpha_{\mathrm{b}} \leq 20^{\circ}$ and $5 \leq \mathrm{x} / \mathrm{d} \leq 13$ for incompressible flow. No extensive body-vortex wake surveys have been conducted in compressible subsonic flow.

The division of vorticity between the primary vortex and the feeding sheet is taken from a correlation of data given in Reference 11. This correlation is represented by

$\frac{\Gamma_{y}}{\Gamma_{t}}=1-0.15(\mathrm{x} / \mathrm{a}) \alpha_{\mathrm{b}}+0.008(\mathrm{x} / \mathrm{a})^{2} \alpha_{\mathrm{b}}^{2}$ where $\Gamma_{\mathrm{t}}^{\prime}=\Gamma_{\mathrm{p}}+\Gamma_{s^{\prime}}$ Although the data from Reference 11: are for supersonic flow, it is reasonable to assume that the ratio of primary vortex strength to total vorticity is the same in subsonic and supersonic flow.

The location of the primary vortex is taken from the experimental data of Grosche, 12 Tinling and Allen, ${ }^{13}$ and Fidler, Nielsen, and Schwind. ${ }^{14}$ The experimental data for the location of the right-hand primary vortex (vortex 1) in polar coordinates are approximated by

$\phi_{\mathrm{i}}=74^{\circ}$

$r_{1} / a=0.70+0.06 \sqrt{M_{\infty}+1}(x / a+6) \sqrt{\alpha_{b}}$.

These equations incorporate the moderate increase in radial location of the vortex center with Mach number measured by Tinling and Allen.

The radius of the vortex core is taken from the data of Reference 11. A fit of the data for angles of attack of $10^{\circ}$ and $15^{\circ}$ and body lengths from 7 to 14 calibers is given by

$$
r_{c} / a=0.030(x / a) \sqrt{\alpha_{b}} .
$$

The computational procedure for the body flow field model will now be discussed. The order of calculation is as follows:

(a) $\alpha_{b}$ and $x$ are set.

(b) $\Gamma_{i}$ is calculated from Eq (?).

(c) $\Gamma_{\mathrm{p}}$ and $\Gamma_{\mathrm{s}}$ are calculated from Eq (10).

(d) $\phi_{1}$ and $r_{1}$ are calculated from Eq (ii).

(e) $r_{c}$ is calculated from $\mathrm{Eq}(12)$.

(f) $\phi_{\mathrm{ss}}$ is computed from Eq (5) by increasing $\phi_{\mathrm{ss}}$ from $0^{n}$ in increments of $0.01 \phi_{1}$ until F.q (5) is satisfied. Recall that $\phi_{\mathrm{ss}}=\phi_{5}$ and $\mathrm{r}_{5}=1.01 \mathrm{a}$.

(g) $\mathrm{L}_{\mathrm{s}}$ is calculated from $\mathrm{Eq}$ ( 6 ) by Simpson's Rule of numerical integration.

(h) $\phi_{j}$ for $j=9,13, \ldots N_{v}-3$ is calculated by increasing $\phi_{j}$ from $\phi_{j-4}$ in increments of $0.01 \psi_{1}$ until the integral equation is satisfied. The integral is evaluated by Simpson's Rule. In the present work, $N_{v}$ is. set at 44 ; that is, 10 vortices in each sheet.

(i) $r_{j}$ for $j=9,13 \ldots N_{v}-3$ is calculated from Eq (8).

(j) $y_{j}, z_{j}$ for $j=1,5,9, \ldots N_{v}-3$ are calculated from $\phi_{j}$, $r_{j}$ using the polar to Cartesian transformation.

(k) $y_{j}, z_{j}$ for all remaining vortices are calculated from $\mathrm{Eq}(3)$.

(l) $v_{c}, w_{c}$ are calculated from Eq (2). 


\section{Lifting Theory}

Various lifting theories were considered for use with the present flow model for the prediction of forces and moments produced by fins. The criteria by which a lifting theory was chosen was that the theory must be able to consider very nonuniform, rotational, approach flow and it must include fin stall and poststall characteristics. These criteria quickly limited the possible theories to strip theory. In strip theory it is assumed that the normal force on a chordwise strip of fin can be calculated by using the local dynamic pressure and.angle of attack of the strip, independent of adjacent chordwise strips. Significant elements included in the present lifting theory are the following: normal force distribution over the lifting surface depends upon fin aspect ratio and leading and trailing edge sweep; individual control deflection of each fin is allowed; fin-fin interference due to both control deflection and rolling rate is included; normal force depends upon free-steam Mach number; and nonuniform approach flow allers the effective leading edge sweep.

\section{a. Local Normal Force}

The local normal force on a differential element of the fin surface is written as (see Figure 3)

$$
d N=C_{n} q d x d r
$$

where $C_{n}$ is the local normal-force coefficient and $q$ is the local dynamic pressure (including. that due to missile rolling speed). The local-normal force coefficient $C_{n}$ is composed of three separate functions: (1) the normal force due to the local angle of attack of the differential element, (2) the local chordwise distribution, and (3) the local spanwise distribution. Assuming a product form of the function, one has

$$
C_{N}=\sigma C_{n}\left(\alpha_{\ell}\right) C(x, r) S(r)
$$

where $\sigma$ is a geometric scaling factor, $\mathrm{C}_{\mathrm{N}}\left(\alpha_{\ell}\right)$ is the local normal force coefficient due to local angle of attack, and $C(x, r)$ and $S(r)$ are the chordwise and spanwise normal force distributions, respectively, for uniform approach flow.

For arbitrary planform fins, it greatly simplifies matters if $C$ and $S$ are written in terms of appropriate fin-oriented coordinates. To determine the appropriate fin-oriented variables, the $x$ coordinate of the leading and trailing edges of the fin is written as

$$
\begin{aligned}
& x_{l e}=x_{1}+\left(x_{2}-x_{1}\right)(r-a) /\left(b_{0}-a\right) \\
& x_{t e}=x_{3}+\left(x_{4}-x_{3}\right)(r-a) /\left(b_{0}-a\right)
\end{aligned}
$$

where $x_{1}, x_{2}, x_{3}$, and $x_{4}$ are defined in Figure 3 , and $b_{0}$ is the semispan of the fin. $x_{2}, x_{3}$, and $x_{4}$ can be related to the leading edge sweep $\Lambda_{\ell \mathrm{e}}$ and the root chord of the fin $c_{r}$ as

$$
\begin{aligned}
& x_{2}=x_{1}+\left(b_{0}-a\right) \tan \Lambda_{l e} \\
& x_{3}=x_{1}+c_{r} \\
& x_{4}=x_{3}+\left(b_{0}-a\right) \tan \Lambda_{l e} .
\end{aligned}
$$

Substituting these equations into Eq (15), one obtains

$$
\begin{aligned}
& x_{l e}=x_{1}+(r-a) \tan \Lambda_{l e} \\
& \dot{x}_{t e}=x_{1}+c_{r}+(r-a) \tan \Lambda_{t e}
\end{aligned}
$$

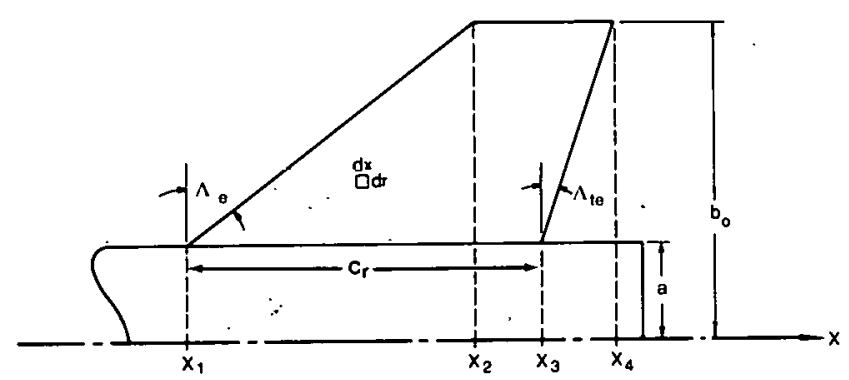

Figure 3. General Planform Fin

Using the boundaries of the fin planform (i.e., $x_{\ell e}$ $x_{t e}, a$, and $b_{0}$ ) as scaling variables, a convenient set of fin-oriented coordinates are

$$
\begin{aligned}
& \xi=\left(x-x_{t e}\right) /\left(x_{l e}-x_{t e}\right) \\
& \eta=(r-a) /\left(b_{o}-a\right)
\end{aligned}
$$

where $\xi$ is the chordwise variable and $\eta$ is the spanwise variable (Figure 4 ).

\section{b. Normal-Force Distribution}

Experimental data ${ }^{15-19}$ for normal-force distribution over the surface of a number of fin planforms was studied in order to arrive at general expressions for $C(\xi)$, and $S(\eta)$. After devising and testing a considerable number of expressions for the chordwise and spanwise distributions, the following equations were adopted

$$
\begin{aligned}
& \mathrm{C}(\xi)=\sqrt{\xi} \exp \left[\xi^{2} / \sqrt{\cos \Lambda_{\ell \mathrm{e}}}\right] \\
& \mathrm{S}(\eta)=\left(1+\eta^{2 \mathrm{~A}_{\mathrm{e}}}\right) \sqrt{1-\eta^{2}}
\end{aligned}
$$


where

$$
\begin{aligned}
A_{e}= & 2\left(b_{o}-a\right)^{2} / S_{f} \\
S_{f}= & \frac{1}{2}\left(b_{o}-a\right)\left[2 c_{r} /\left(b_{o}-a\right)\right. \\
& \left.-\tan \Lambda_{\ell e}+\tan \Lambda_{t e}\right] .
\end{aligned}
$$

$A_{e}$ is the exposed aspect ratio; that is, the aspect ratio of the lifting surface formed by eliminating the body and placing the root chords of two adjacent fins together. $S_{f}$ is the planform area of a single fin. Equations (19a) and (19b) have been shown to give valid results for exposed aspect ratios from 0.5 to 5 , leading edge sweep from $0^{\circ}$ to $80^{\circ}$, and trailing edge sweep from $-60^{\circ}$ to $80^{\circ}$.

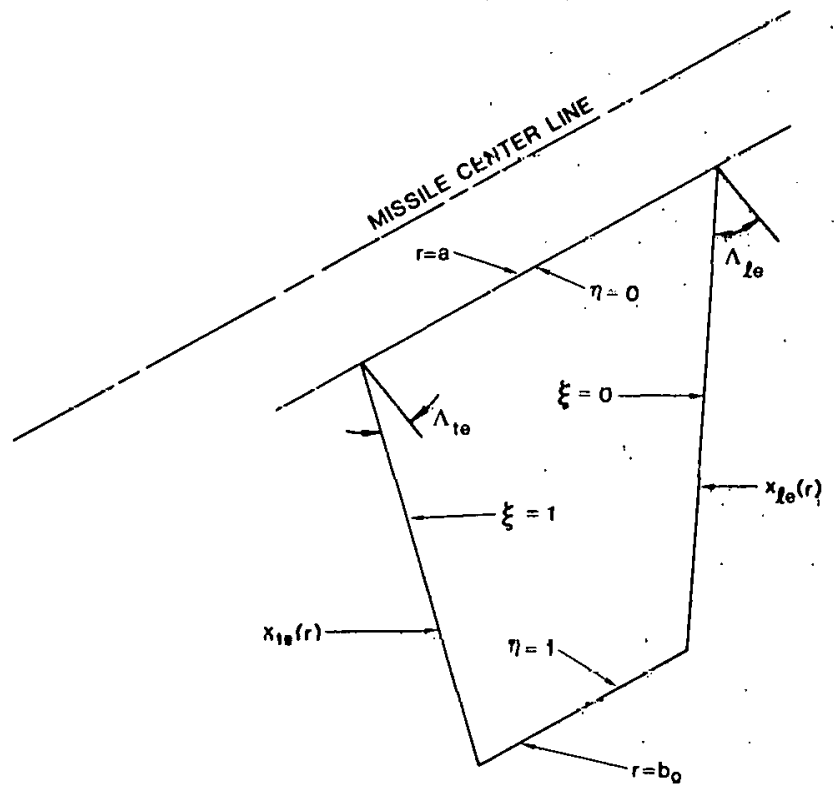

Figure 4. Fin-Oriented Coodinates $\xi, \eta$

The normal force distribution over the surface of the fin for uniform approach flow is then provided by the product of Eqs (19a) and (19b). This expression was compared qualitatively with experimental data by means of a three-dimensional computer graphics routine, DISSPLA. * This routine provided a means of visualizing the normal force distribution over the surface of the fin. Shown in Figures 5 through 9 are sets of three-dimensional perspective plots of typical planforms that were examined. Figure 5 shows a

*DISSPLA is a graphics of software package available from Integrated Software Systems Corporation, San Diego, CA. sequence of untappered fins with $\Lambda_{l \mathrm{e}}=\Lambda_{\mathrm{te}}=60^{\circ}$ for $A_{e}=0.5,1$, and 3 . The view in the perspective plot is from behind and above the fin surface, looking upstream and slightly inboard. The graphics routine uses a rectangular area over which the surface function is defined. Consequently, the regions that show a surface value of zero are not part of the fin planform. For example, on Figure 5 the trailing edge of the fin is located at the junction of the nonzero and zero surface values. Another point to note on the perspective plots is a "spike" character at the leading edge of the planform. This characteristic is not inherent in the equations but simply is a result of the mesh size on the surface and the zero value of the surface just ahead of the leading edge.

Figure 6 shows a sequence of fins with $\Lambda_{t e}=60^{\circ}$, $\Lambda_{t e}=20^{\circ}$ for $A_{e}=0.5,1$, and 2.92. In Figure 6 the taper ratio decreases until in Figure $6 \mathrm{c}$ an arrow wing is achieved. Note in this sequence of figures that as the leading edge becomes longer the normal force loading reflects the very high loading near the leading edge caused by the increasing strength of the leading edge vortex.

Figure 7 shows a rectangular planform for $A_{e}=$ $0.5,1 .$, and 3. For the low aspect ratio planform (Figure 7a), note the increase in normal force near the tip chord due to the tip vortex increasing in strength along the tip chord. For the high aspect ratio planform (Figure $\overline{7} \mathrm{c}$ ), it can be seen that the spanwise load distribution nears the classical elliptic loading.

Figure 8 presents the loading for $\Lambda_{\ell t}=60^{\circ}, \Lambda_{\mathrm{te}}=$ $0^{\circ}$, and $A_{e}=0.5,1$, and 2.31. Figures $8 a$ and $8 b$ show clipped deltas, and Figure $8 \mathrm{c}$ chows a delta planform. When the distribution for the delta planform is compared with experimental data, ${ }^{18}$ it is seen that the empirical equation models the data except near the leading edge.

Figure 9 shows a trapezoid planform with decreasing taper ratio, $\Lambda_{\ell \mathrm{e}}=-\Lambda_{\mathrm{tc}}=40^{\circ}$ and $\mathrm{A}_{\mathrm{e}}=0.5,1$., and 2.38. The planform with $A_{e}=2.38$ (Figure $8 \mathrm{c}$ ) has a taper ratio of zero and is, therefore, a diamond planform.

The geometric scaling factor in $\mathrm{Eq}$ (14) can now be determined. $\sigma$ is evaluated by the requirement that the integrated average of the assumed normal force distribution over the surface of the fin must be unity; i.e., the assumed normal force loading must be normalized. Therefore, one may write

$$
q_{\infty} \int_{\text {fin }} \int_{n} d A=q_{\infty} C_{N} s_{f}
$$


Substituting $C_{n}$ from Eq (14), one has

$\sigma=\int_{a}^{b_{0}} \int_{x_{\ell e}}^{x_{t e}} C(x, r) S(r) d x d r=S_{f}$.

Transforming to the fin coordinates $\xi, \eta$ (Eq 18) and solving for $\sigma$, one obtains

$\sigma=\frac{\mathrm{S}_{\mathrm{f}}}{\left(\mathrm{b}_{\mathrm{o}}-\mathrm{a}\right) \int_{0}^{1} \int_{0}^{1} \Omega(\eta) \mathrm{C}(\xi) \mathrm{S}(\eta) \mathrm{d} \xi \mathrm{d} \eta}$

where

$\Omega(\eta)=c_{r}-\left(b_{o}-a\right)\left(\tan \Lambda_{\ell \mathrm{e}}-\tan \Lambda_{\mathrm{te}}\right) \eta$

and $C(\xi)$ and $S(\eta)$ are given by Eq (19).

\section{c. Local Angle of Attack}

Referring back to Eq (14), $C_{N}$ depends on the local angle of attack of the chordwise strip. The local angle of attack is calculated by using the unit normal vector of the fin surface, $\vec{n}$, and the total velocity, $\vec{V}$ (Figure 10). The geometric local angle of attack can be shown to be

$\alpha_{\ell}=\sin ^{-1}[\overrightarrow{\mathrm{n}} \cdot \overrightarrow{\mathrm{V}} /(|\overrightarrow{\mathrm{n}}| \overrightarrow{\mathrm{V}} \mid)]$.

The surface normal vector of the fin depends on the roll angle $\phi$ and the control deflection of each fin $\delta_{j}, j$ $=1,2,3,4$. Let the sign convention of the control deflection of each fin be as follows: positive control deflection of Fins 1 and 3 produces a positive normal force, i.e., a pitch down maneuver; positive control deflection of Fins 2 and 4 produces a positive side force, i.e., a yaw left maneuver (see Figure 10). The sign convention for the surface normal vector, however, is such that the vector always points in the counterclockwise direction. Referring to Figure 10, the surface-normal vector is

$\vec{n}=\sin \left(\Delta \delta_{j}\right) \vec{i}-\cos \left(\delta_{j}\right) \sin \phi \vec{j}+\cos \left(\delta_{j}\right) \cos \phi \vec{k}$

where

$\Delta=\cos \phi /|\cos \phi|$

$\Delta$ simply provides the sign of $\delta_{\mathrm{j}}$ which is consistent with the above-mentioned sign convention.
The local total velocity (i.e., the velocity of the fluid relative to the chordwise strip) is composed of two types of terms: the fluid velocity relative to the fixed coordinate system and the velocity of the fixed coordinate system relative to the spinning chordwise strip. Therefore, using Eq (1) it can be written

$$
\begin{gathered}
\overrightarrow{\mathrm{V}}=\mathrm{U}_{\infty} \cos \alpha_{\mathrm{b}} \overrightarrow{\mathrm{i}}+\left(\mathrm{v}_{\mathrm{c}}+\dot{\phi} \mathrm{r} \sin \phi\right) \overrightarrow{\mathrm{j}} \\
+\left(\mathrm{w}_{\mathrm{c}}-\dot{\phi} \mathrm{r} \cos \phi\right) \overrightarrow{\mathrm{k}}
\end{gathered}
$$

where $\mathrm{v}_{\mathrm{c}}$ and $\mathrm{w}_{\mathrm{c}}$ are given by $\mathrm{Eq}(2)$ and $\dot{\phi}$ is the roll rate of the missile.

The axial location at which the flow model is evaluated is calculated from the pin planform characteristics. The axial location is chosen as the average quarter chord location of the root and tip chords; i.e.,

$$
x_{v}=\left(x_{1}+0.25 c_{r}+x_{2}+0.25 c_{t}\right) / 2
$$

where $x_{v}$ is the axial location of the vortex model and $c_{t}$ is the length of the tip chord. Rewriting this equation, one has

$$
x_{v}=x_{1}+\left(0.25 c_{r}+0.25 c_{t}+\left(b_{o}-a\right) \tan \Lambda_{e}\right) / 2 .
$$

This axial location is used in Eqs (9) through (12).

The geometric local angle of attack can now be calculated by substituting Eqs (22) and (23) into Eq (21). Interference between fins, however, will alter $\alpha_{\ell}$ for control deflection and a rolling missile. These interference effects are considered in the paragraph entitled Roll Moment.

\section{d. Effective Aspect Ratio}

Consider an aerodynamic effect that occurs when the lifting surface is attached to the missile body. If the body is at high angle of attack, the angle in the plane of the fin between the approach flow and the fin leading edge can vary significantly, depending on the roll angle of the body; that is, the fin is yawed with respect to the approach flow for various roll angles around the body. This yaw angle results in an effective change in the leading and trailing edge sweep and effective aspect ratio of the fin. A simple example of this is to consider Fin 4 at a roll angle of $0^{\circ}$ with the body at angle of attack $\alpha_{\mathrm{b}}$ (see Figure 10). The effective leading edge sweep of Fin 4 at $\phi=0^{\circ}$ then is approximately $\Lambda_{\ell \mathrm{e}}-\alpha_{\mathrm{b}}$. If $\Lambda_{\ell \mathrm{e}}=0^{\circ}$, then the fin at this roll angle would actually be swept forward. 


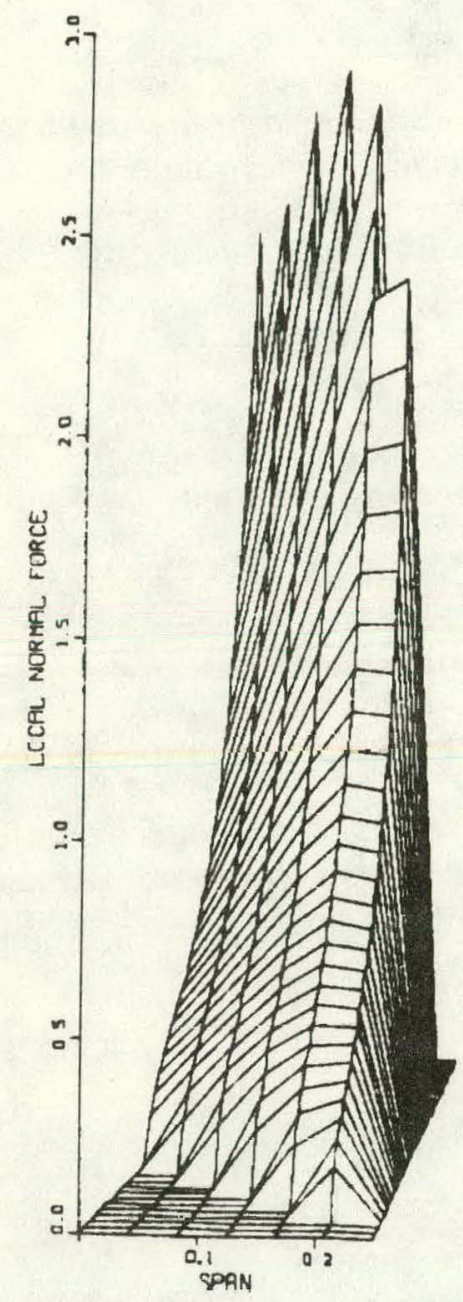

a) $\mathrm{A}_{\mathrm{e}}=.5$

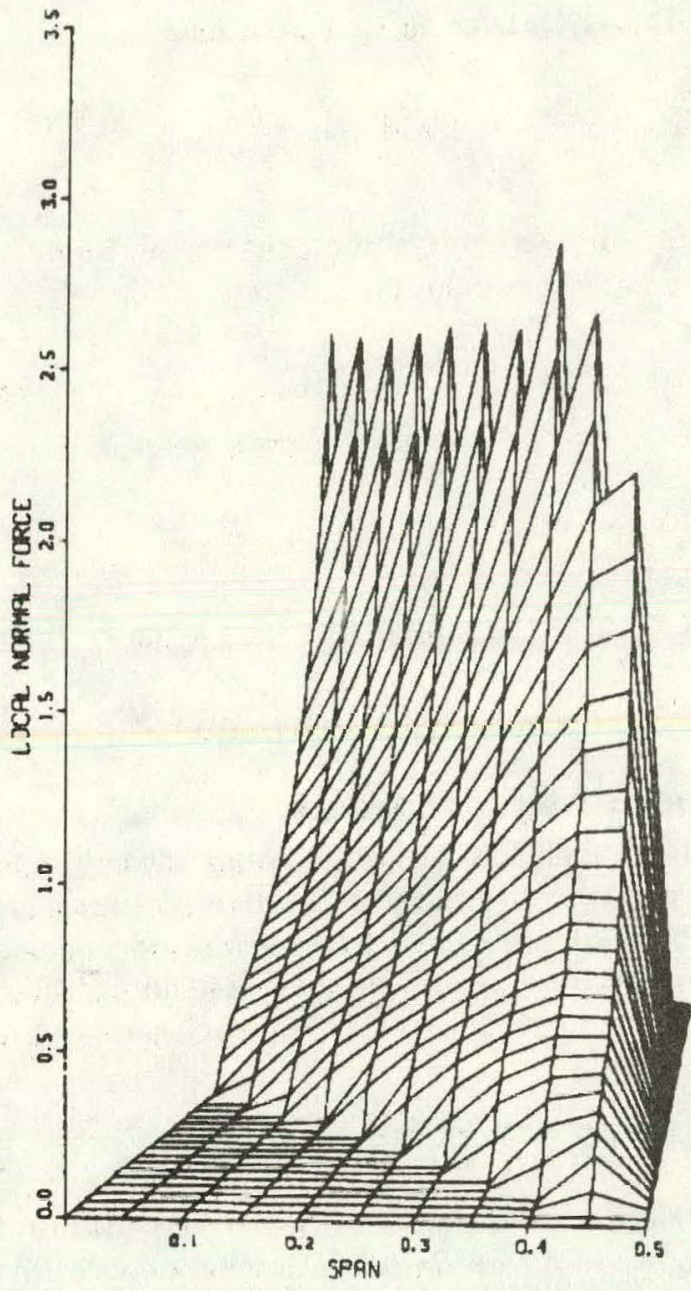

b) $A_{e}=1$.

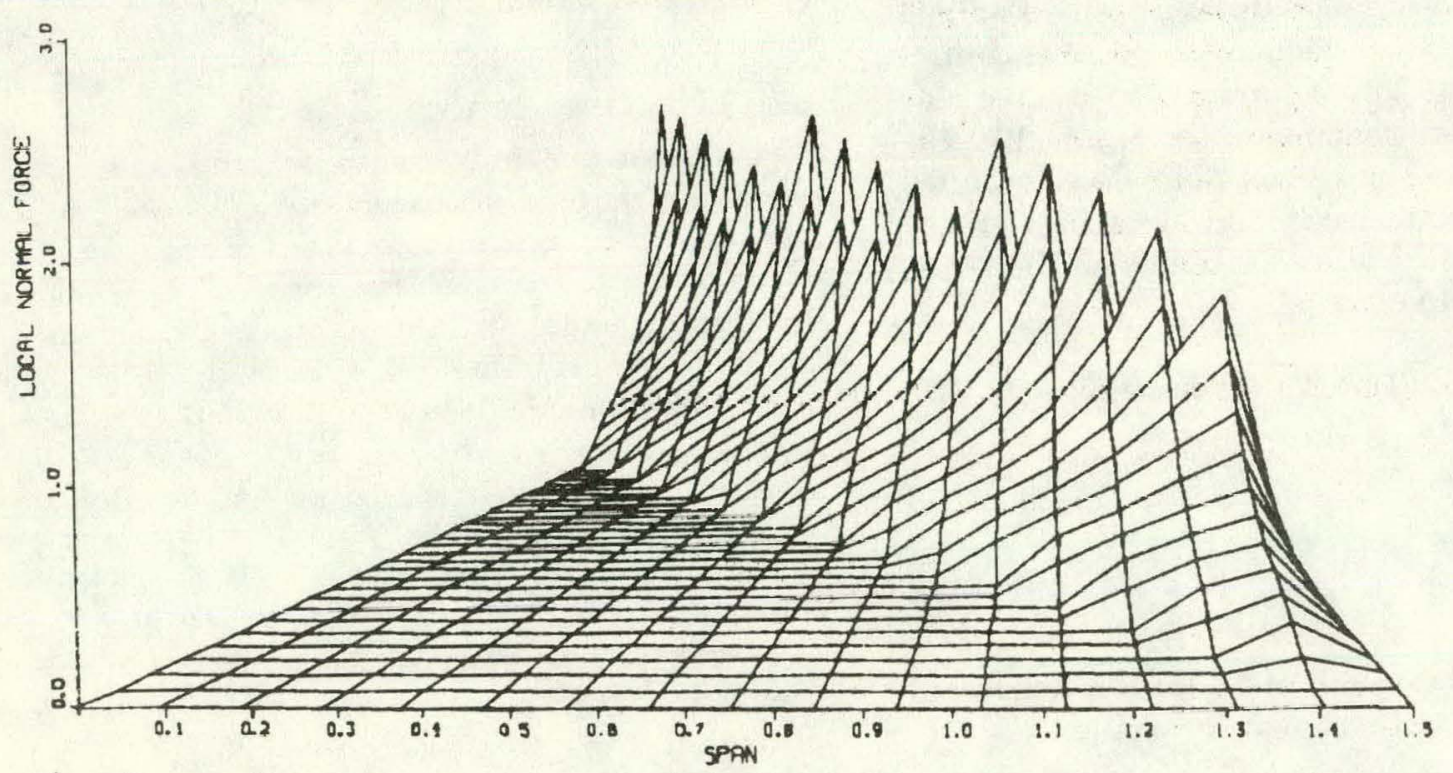

C) $A_{e}=3$.

Figure 5. Normal Force Distribution Over Fin Surface for $\Lambda_{\ell \mathrm{e}}=\Lambda_{\mathrm{te}}=60^{\circ}$ 


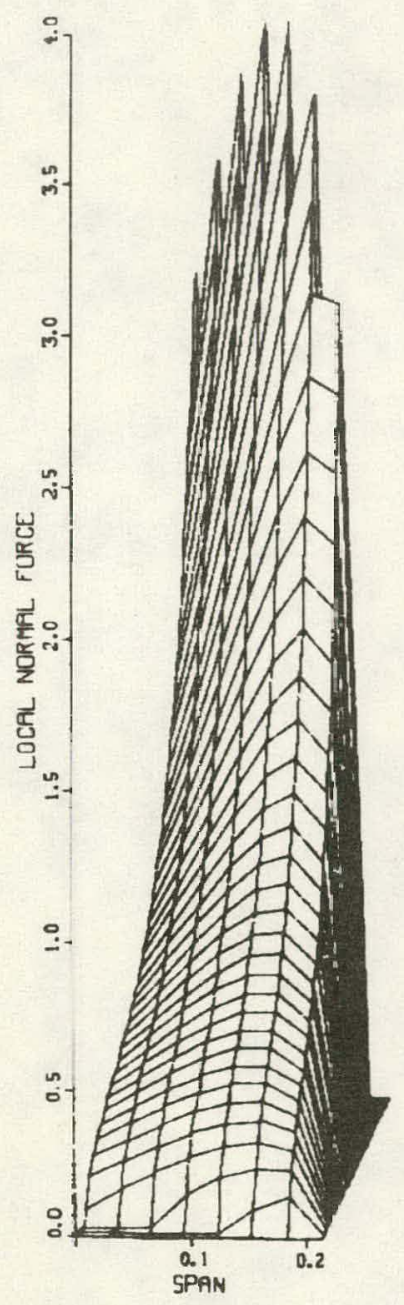

a) $\mathrm{A}_{\mathrm{e}}=.5$

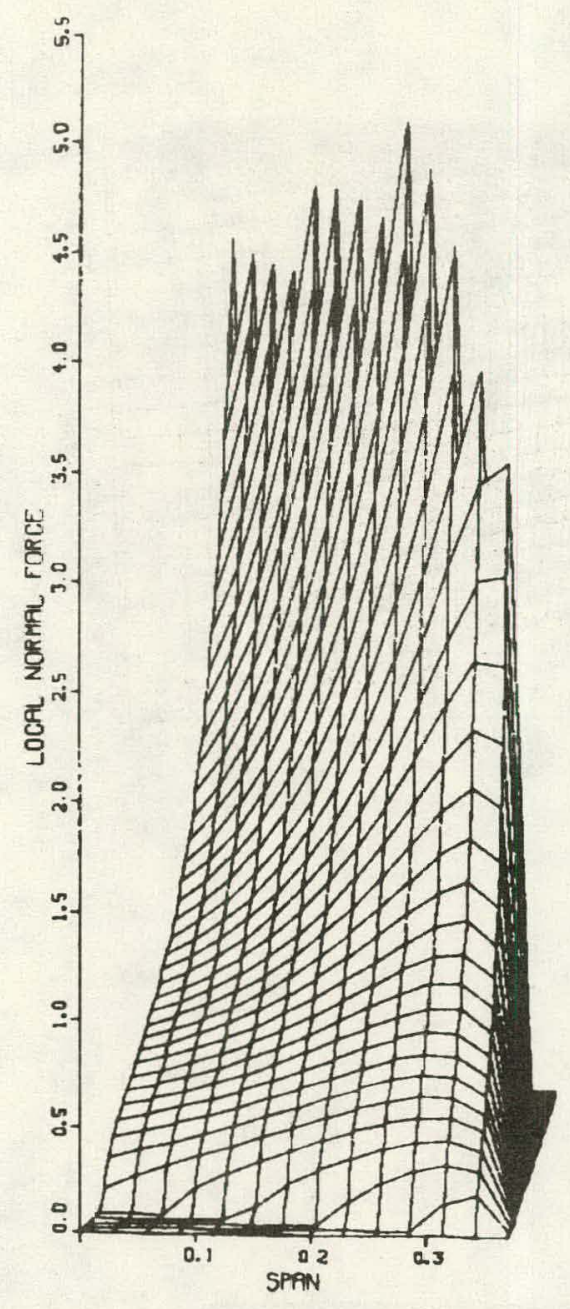

b) $A_{e}=1$.

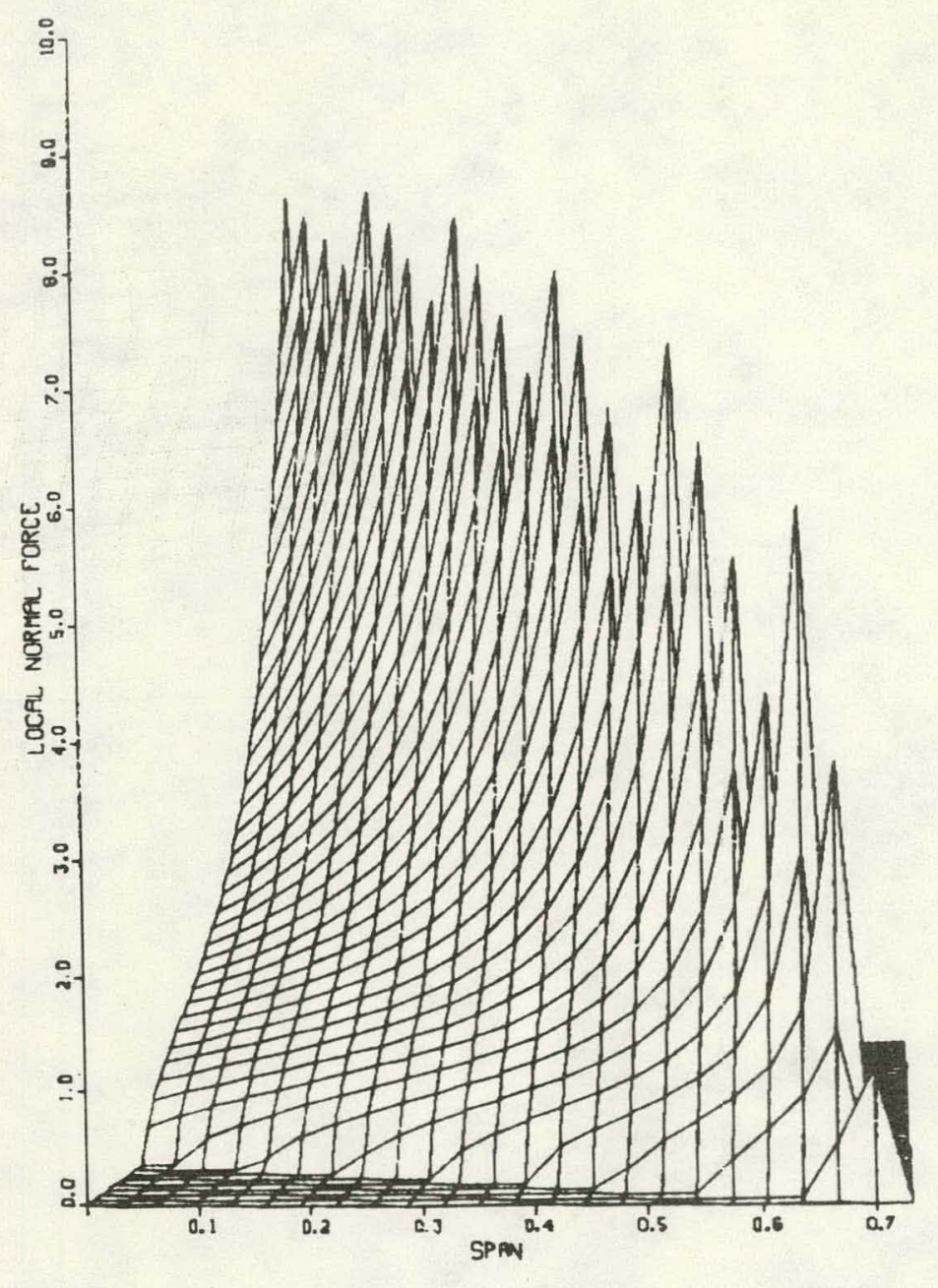

c) $\mathrm{A}_{\mathrm{e}}=2.92$

Figure 6. Normal Force Distribution Over Fin Surface for $\Lambda_{\ell \mathrm{e}}=60^{\circ}$ and $\Lambda_{\mathrm{te}}=20^{\circ}$ 


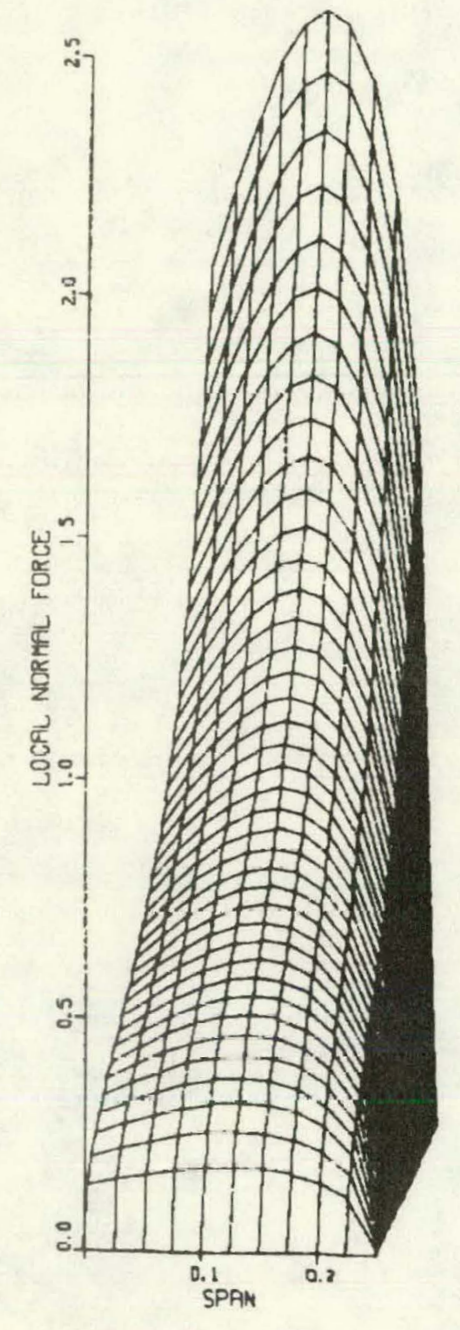

a) $\mathrm{A}_{e}=.5$

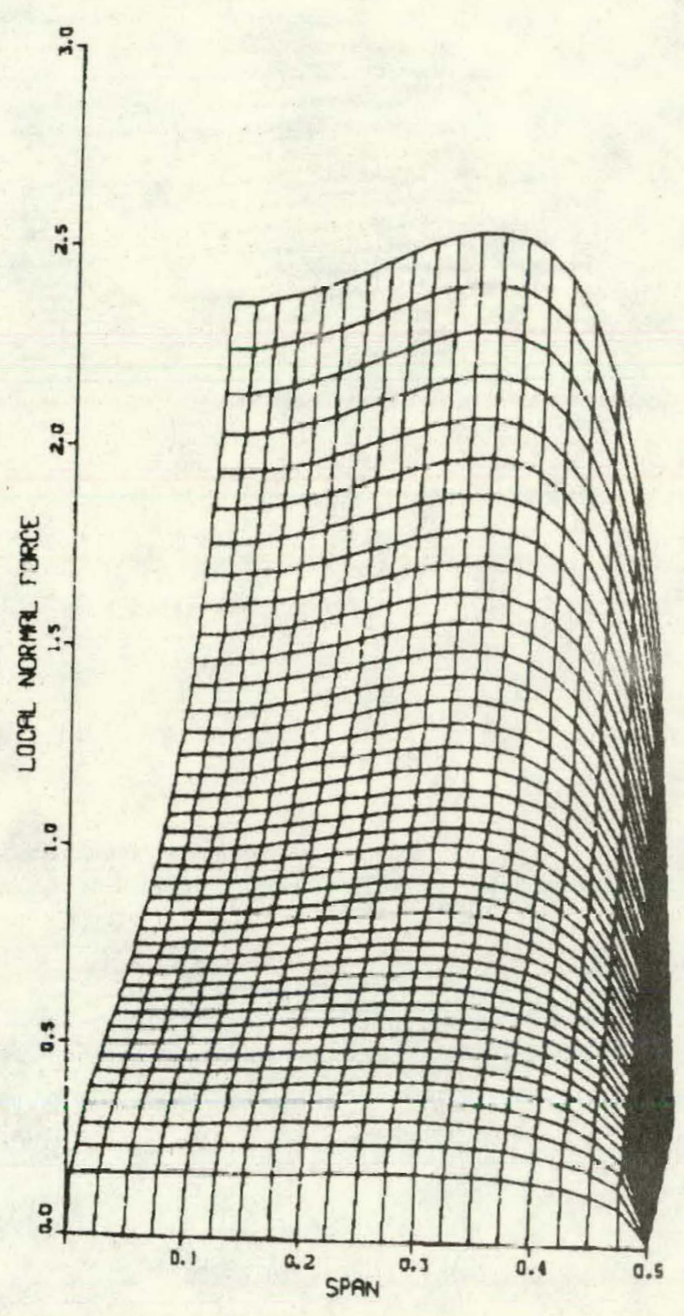

b) $A_{C}=1$

Figure 7. Normal Force Distribution Over Fin Surface for $\Lambda_{\ell e}=\Lambda_{t e}=0^{\circ}$ 


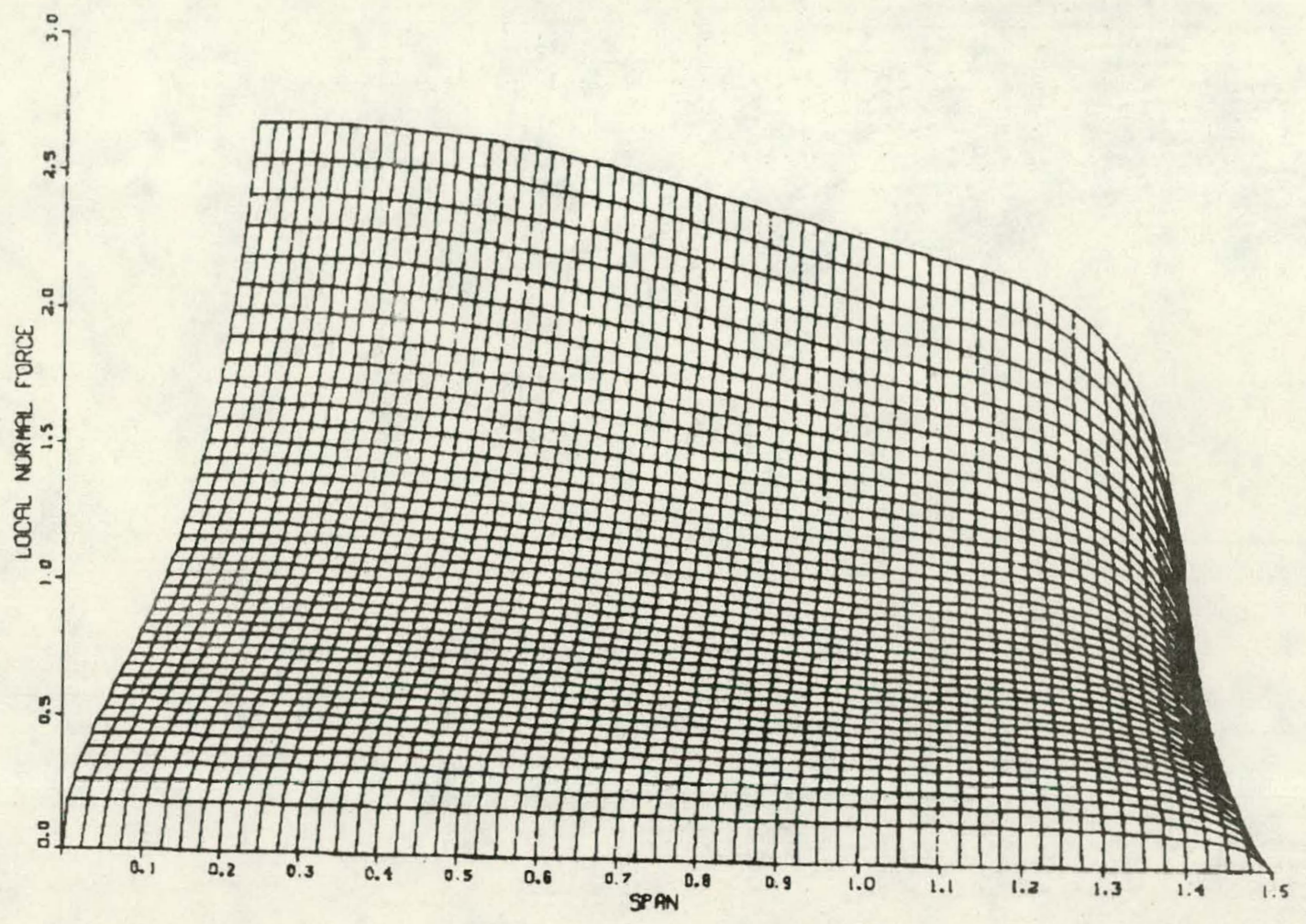

c) $A_{e}=3$.

Figure 7. (Concluded) 

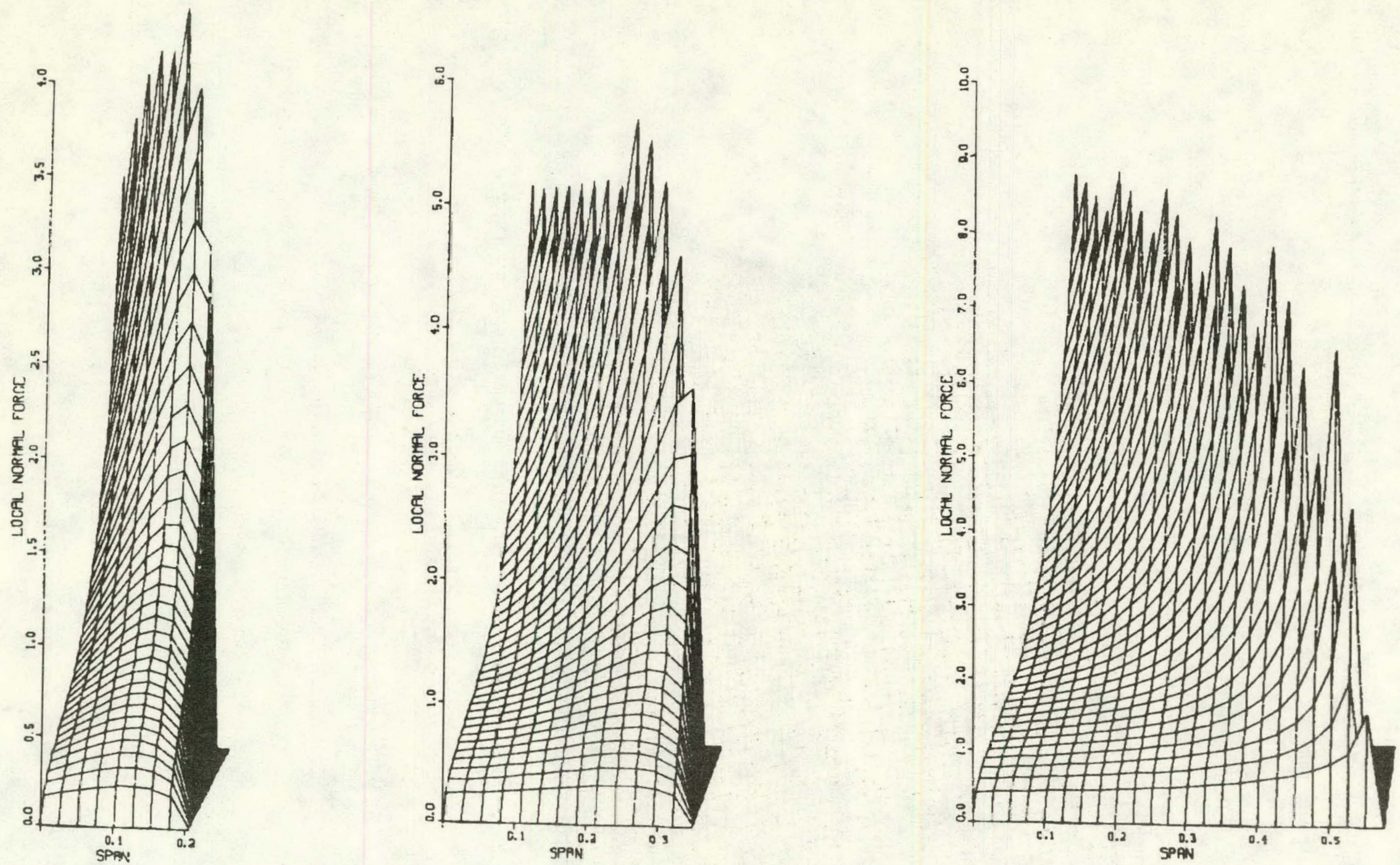
a) $\mathrm{A}_{\mathrm{e}}=.5$
b) $A_{e}=1$.

c) $A_{e}=2.31$

Figure 8. Normal Force Distribution Over Fin Surface for $\Lambda_{\ell}=60^{\circ}$ and $\Lambda_{t e}=0^{\circ}$ 


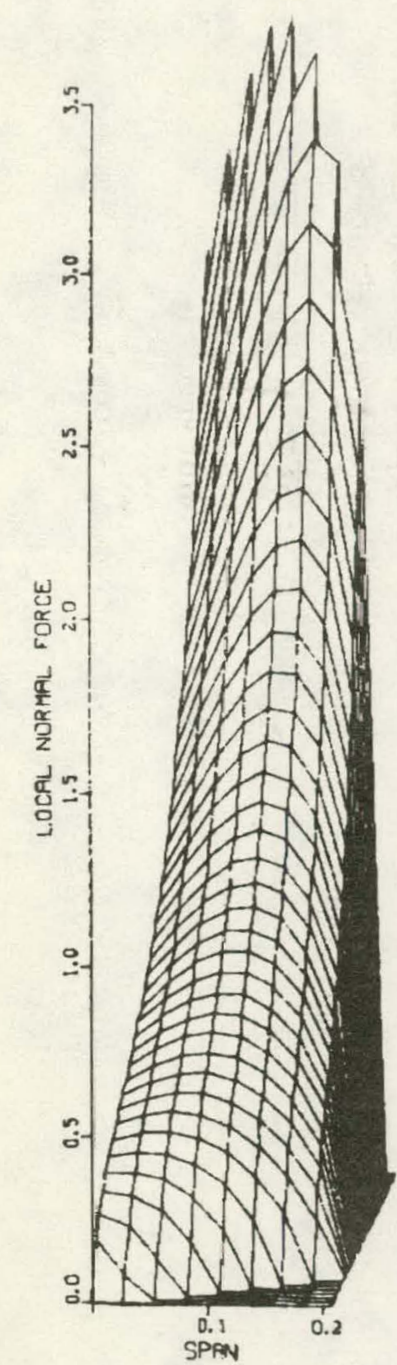

a) $A_{e}=.5$

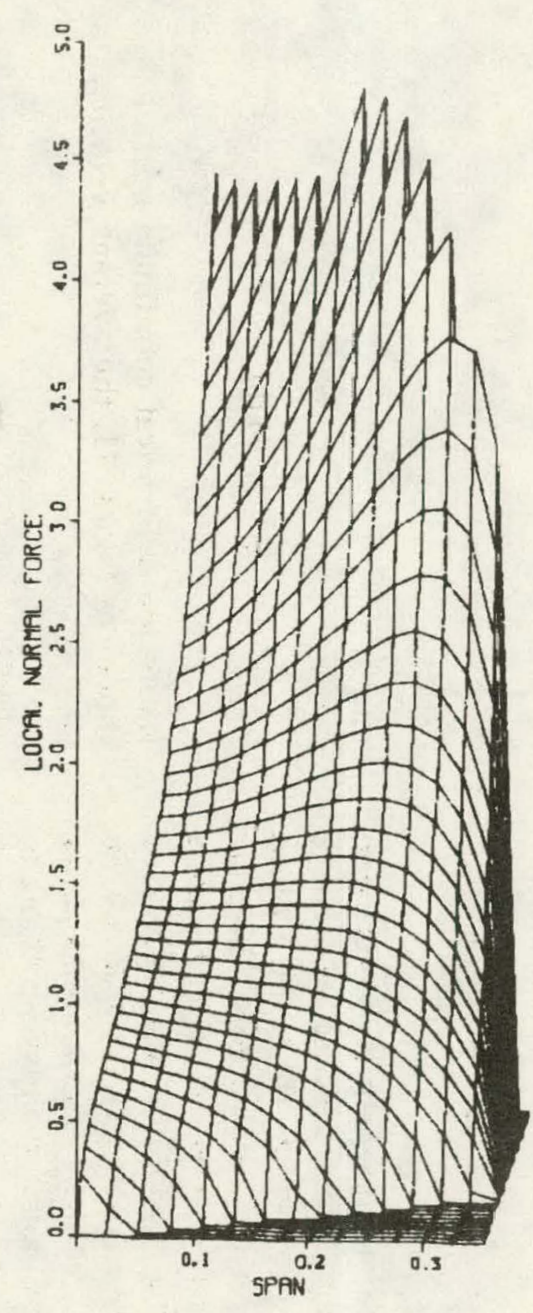

b) $A_{e}=1$

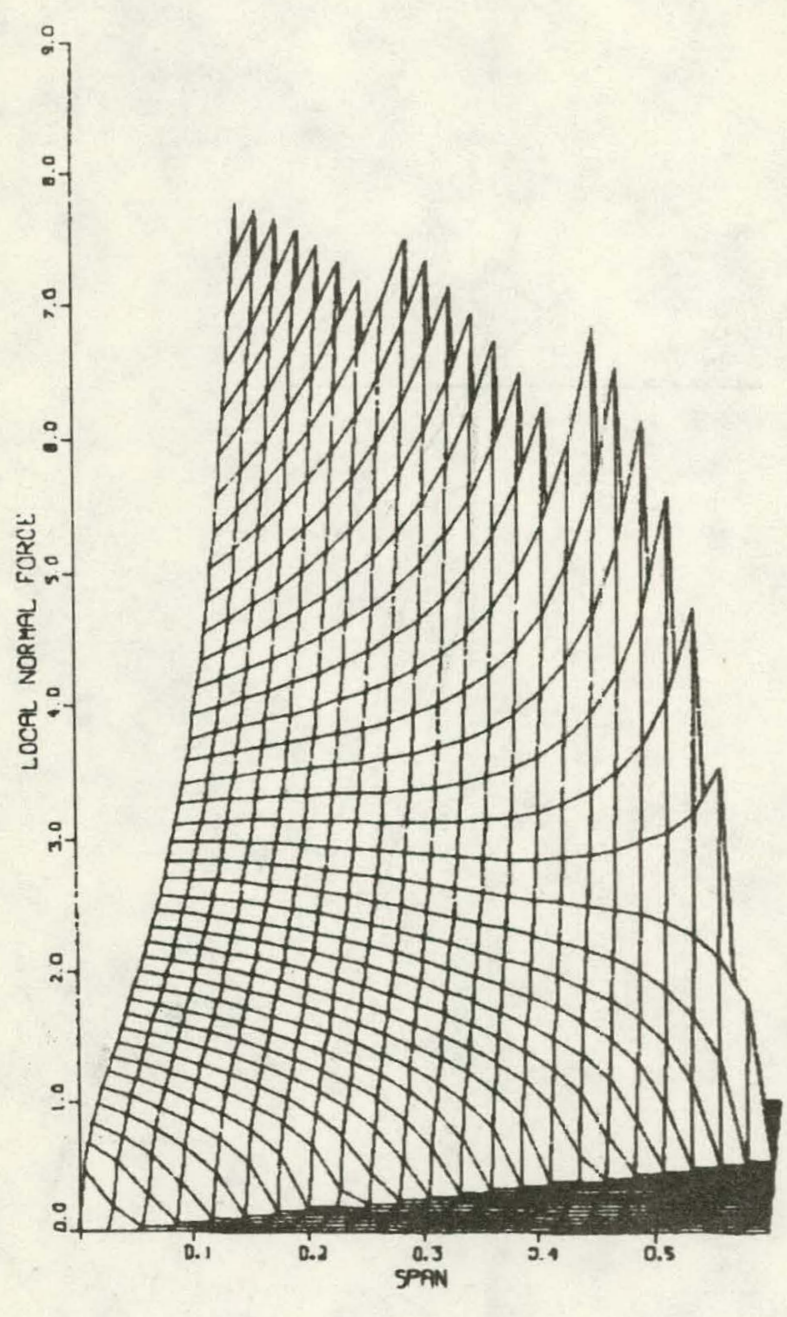

c) $\mathrm{A}_{\mathrm{e}}=2.38$

Figure 9. Normal Force Distribution Over Fin Surface for $\Lambda_{\ell \mathrm{e}}=40^{\circ}$ and $\Lambda_{\mathrm{te}}=-40^{\circ}$ 


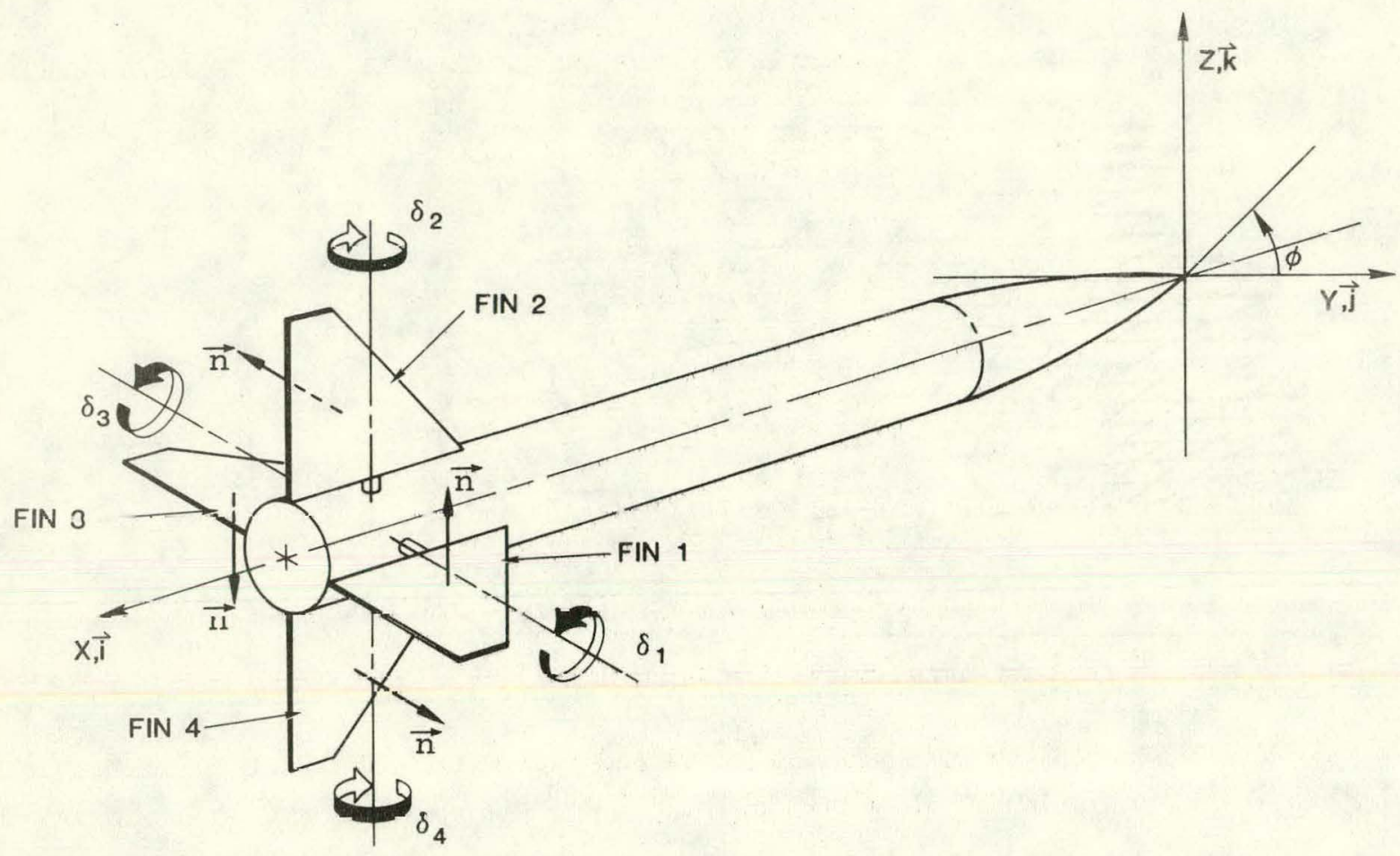

Figure 10. Surface Normal Vector and Sign Convention for Fin Control Deflection

The effective leading edge sweep, $\hat{\Lambda}_{\ell e^{\prime}}$ and effective trailing edge sweep $\hat{\Lambda}_{\mathrm{te}}$, are derived by relating the average flow velocity along the fin to the leading and trailing edge unit vectors, respectively. The most convenient coordinate system to use is cylindrical coordinates with the orthogonal unit vectors located in the plane of the fin (see Figure 11). Let $\bar{v}_{c}$ and $\bar{w}_{c}$ be the dverage cross-flow velocity components along the exposed semispan of the fin at a given roll angle; then,

$$
\bar{v}_{c}=\frac{1}{b_{o}-a} \int_{a}^{b_{o}} v_{c}(r, \phi) d r
$$

and

$$
\bar{w}_{c}-\frac{1}{b_{0}-a} \int_{a}^{b_{0}} w_{c}(r, \phi) d r
$$

where $v_{c}$ and $w_{c}$ are given by Eq (2). Rewriting these equations in terms of fin-oriented coordinates, one has

$$
\overline{\mathrm{v}}_{\mathrm{c}}=\int_{\mathrm{a}}^{1} \mathrm{v}_{\mathrm{c}}(\eta, \phi) \mathrm{d} \eta
$$

and

$$
\overline{\mathrm{w}}_{\mathrm{c}}=\int_{0}^{1} \mathrm{w}_{\mathrm{c}}(\eta, \phi) \mathrm{d} \eta .
$$

Using the cylindrical conrdinate unit vectors $\overrightarrow{\mathrm{e}}_{\mathrm{x}}, \overrightarrow{\mathrm{c}}_{\mathrm{r}}$ shown in Figure 11, the average velocity alnng the leading edge is

$$
\vec{V}=\vec{u} \vec{e}_{x}+\vec{v}_{r} \vec{e}_{r}
$$

where

$$
\begin{aligned}
& \overline{\mathrm{u}}=\mathrm{U}_{\infty} \cos \alpha_{\mathrm{b}} \\
& \overline{\mathrm{v}}_{\mathrm{r}}-\overline{\mathrm{v}}_{\mathrm{c}} \cos \phi \quad \mid \overline{\mathrm{w}}_{\mathrm{c}} \sin \phi .
\end{aligned}
$$

Since the sweep angle is measured from a line perpendicular to the approach velocity, the unit vector perpendicular to the average velocity is

$$
\vec{v}_{\perp}=\bar{v}_{r} / \sqrt{\bar{u}^{2}+\bar{v}_{r}^{2}} \vec{e}_{x}+\bar{u} / \sqrt{\bar{u}^{2}+\bar{v}_{r}^{2}} \vec{e}_{r} \text {. }
$$

The leading edge unit vector is

$$
\overrightarrow{\mathrm{e}}_{\ell \mathrm{e}}=\sin \Lambda_{\ell \mathrm{e}} \overrightarrow{\mathrm{e}}_{\mathrm{x}}+\cos \Lambda_{\ell \mathrm{e}} \overrightarrow{\mathrm{e}}_{\mathrm{r}} \text {. }
$$




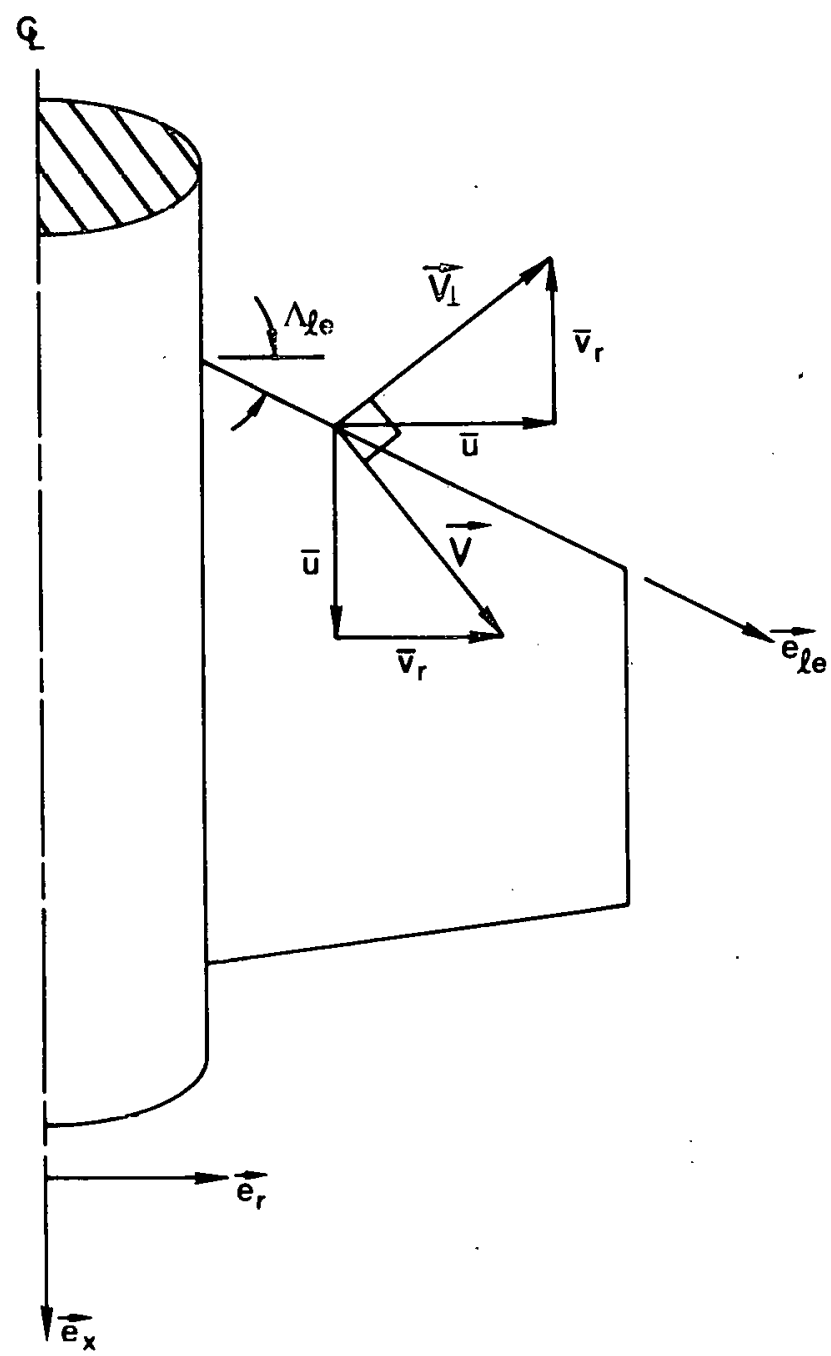

Figure 11. Fin Surface Cylindrical Coordinates

The effective leading edge sweep angle is the angle between $\vec{v}_{\perp}$ and $\vec{e}_{\ell \mathrm{e}}$. Using the scalar product, one obtains

$$
\hat{\Lambda}_{\ell \mathrm{e}}=\cos ^{-1}\left(\overrightarrow{\mathrm{V}}_{\perp} \cdot \overrightarrow{\mathrm{e}}_{\ell \mathrm{e}}\right) \text {. }
$$

Substituting in Eqs (25) and (26),

$\hat{\Lambda}_{\ell \mathrm{e}}=\cos ^{-1}\left[\left(\overline{\mathrm{u}} \cos \Lambda_{\ell \mathrm{e}}-\overline{\mathrm{v}}_{\mathrm{r}} \sin \Lambda_{\ell \mathrm{e}}\right) / \sqrt{\overline{\mathrm{u}}^{2}+\overline{\mathrm{v}}_{\mathrm{r}}^{2}}\right]$.

This expression presents difficulties in evaluation because the arc cosine function is double valued. This problem can be nicely circumvented by noting that the argument of the arc cosine function suggests $\bar{\Lambda}_{l \mathrm{e}}$ might be split into two angles. Let

$$
\hat{\Lambda}_{\ell \mathrm{e}}=\Lambda_{\ell \mathrm{e}}+\epsilon_{\ell \mathrm{e}}
$$

where $\epsilon_{\ell \mathrm{e}}$ is the deviation of the effective leading edge sweep from the geometric leading edge sweep. Now Eq (27) can be written as

$$
\begin{gathered}
\cos \left(\Lambda_{\ell \mathrm{e}}+\epsilon_{\ell \mathrm{e}}\right)=\frac{\ddot{\mathrm{u}}}{\sqrt{\overline{\mathrm{u}}^{2}+\overline{\mathrm{v}}_{\mathrm{r}}^{2}}} \cos \Lambda_{\ell \mathrm{e}} \\
-\frac{\overline{\mathrm{v}}_{\mathrm{r}}}{\sqrt{\overline{\mathrm{u}}^{2}+\overline{\mathrm{v}}_{\mathrm{r}}^{2}}} \sin \Lambda_{\ell \mathrm{e}} .
\end{gathered}
$$

Using the trigonometric identity for the cosine of a sum, one has

$$
\begin{aligned}
& \cos \epsilon_{\ell \mathrm{e}} \cos \Lambda_{\ell \mathrm{e}}-\sin \epsilon_{\ell \mathrm{e}} \sin \Lambda_{\ell \mathrm{e}}= \\
& \frac{\overline{\mathrm{u}}}{\sqrt{\overline{\mathrm{u}}^{2}+\overline{\mathrm{v}}_{\mathrm{r}}^{2}}} \cos \Lambda_{\ell \mathrm{e}}-\frac{\overline{\mathrm{v}}_{\mathrm{r}}}{\sqrt{\overline{\mathrm{u}}^{2}+\overline{\mathrm{v}}_{\mathrm{r}}^{2}}} \sin \Lambda_{\ell \mathrm{e}} .
\end{aligned}
$$

In matching terms, one notes that two mathematically equivalent expressions can be written for $\epsilon_{\ell \mathrm{e}}$. The one involving the cosine function, ,-however, suffers from the same difficulty mentioned above; therefore use

$$
\sin \epsilon_{\ell \mathrm{e}}=\overline{\mathrm{v}}_{\mathrm{r}} / \sqrt{\overline{\mathrm{u}}^{2}+\overline{\mathrm{v}}_{\mathrm{r}}^{2}} .
$$

Substituting this into $\mathrm{Eq}(28)$, one finally obtains

$$
\hat{\Lambda}_{\ell \mathrm{e}}=\Lambda_{\ell \mathrm{e}}+\sin ^{-1}\left(\overline{\mathrm{v}}_{\mathrm{r}} / \sqrt{\overline{\mathrm{u}}^{2}+\overline{\mathrm{v}}_{\mathrm{r}}^{2}}\right) \text {. }
$$

Using exactly the same procedure, the effective trailing edge sweep is given by

$$
\hat{\Lambda}_{\mathrm{te}}=\Lambda_{\mathrm{te}}+\sin ^{-1}\left(\overline{\mathrm{v}}_{\mathrm{r}} / \sqrt{\overline{\mathrm{u}}^{2}+\overline{\mathrm{v}}_{\mathrm{r}}^{2}}\right) .
$$

The effective leading and trailing edge sweep caused by high angle of attack results in an effective aspect ratio of the fin. This is significant since the local normal force coefficient due to angle of attack, $\mathrm{C}_{\mathrm{N}}\left(\alpha_{\ell}\right)$, will now reflect effective changes in fin geometry due to apparent yaw of the fin. To derive an expression for $\hat{A}_{e}$, begin with the definition of $A_{e}$

$$
A_{e}=2\left(b_{o}-a\right)^{2} / S_{f}
$$

where $S_{f}$ is the exposed planform area of a fin. Writing this in terms of fin semispan, root chord, and leading and trailing edge sweep, one has

$A_{e}=4 /\left[2 c_{r} /\left(b_{o}-a\right)-\tan \Lambda_{\ell e}+\tan \Lambda_{t e}\right]$. 
Referring to Figure 12, it can be seen that the exposed fin semispan and root chord also effectively change. It can be shown that

$$
\begin{aligned}
& \hat{b}_{0}-a=\left(b_{o}-a\right) \cos \hat{\Lambda}_{\ell \mathrm{e}} / \cos \Lambda_{\ell \mathrm{e}} ; \\
& \hat{c}_{r}=c_{\mathrm{r}} \cos \Lambda_{\mathrm{te}} / \cos \left(\hat{\Lambda}_{\ell \mathrm{e}}-\Lambda_{\ell \mathrm{e}}+\Lambda_{\mathrm{te}}\right) .
\end{aligned}
$$

q

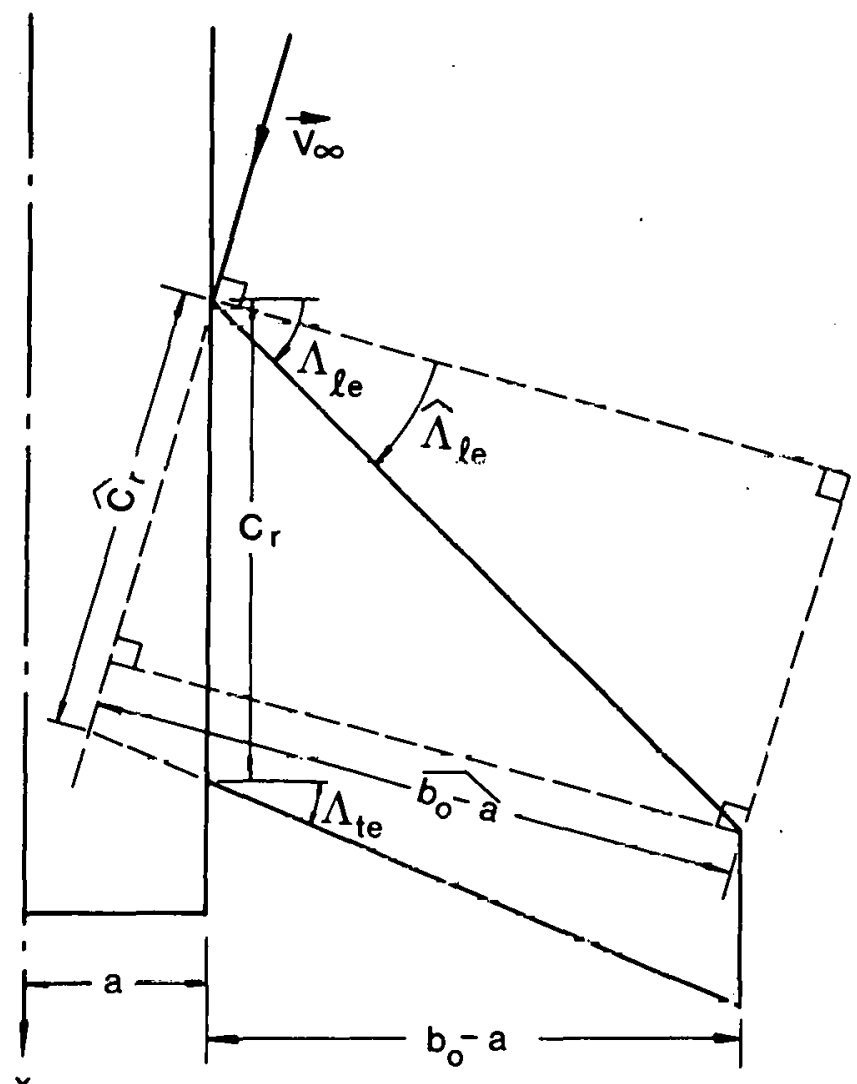

Figure 12. Geometry for Determining Effective Aspect Ratio

Using Eqs (30) and (31), the effective aspect ratio is written as

$$
\dot{A}_{\mathrm{e}}=\frac{4}{\frac{2 c_{\mathrm{r}} \cos \Lambda_{\mathrm{ec}} \cos \Lambda_{\mathrm{te}}}{\left(\mathrm{b}_{\mathrm{e}}-\mathrm{a}\right) \cos \hat{\Lambda}_{\mathrm{ee}} \cos \left(\hat{\Lambda}_{\mathrm{le}}-\Lambda_{\mathrm{le}}+\Lambda_{\mathrm{te}}\right)}-\tan \hat{\Lambda}_{\mathrm{te}}+\tan \hat{\Lambda}_{\mathrm{te}}} .
$$

\section{e. Expression for $\mathbf{C N}_{\mathbf{N}}\left(\alpha_{\ell}\right)$}

The functional dependence of $C_{N}$ on the local angle of attack is given by an empirical expression based on lifting surfaces in uniform approach flow. Other investigators (for example, Reference 6) have used experimental data for $C_{N}\left(\alpha_{\ell}\right)$ directly in their analysis. This is rarely an appealing approach because it requires vast amounts of data to construct a data base sufficiently general to address general planforms over a large range of Mach numbers. Also, this is not possible in the present approach because the effective geometry of the fin (discussed earlier) changes with the character of the nonuniform approach flow.

As the local angle of attack on a missile fin can be approximately $40^{\circ}$ to $50^{\circ}$ when the angle of attack of the body is $25^{\circ}$, the expression for $\mathrm{C}_{\mathrm{N}}\left(\alpha_{\ell}\right)$ must include prestall, stall, and poststall characteristics. The prestall expression for $C_{N}\left(\alpha_{\ell}\right)$ is taken from the work of Lecat and Rietschlin. ${ }^{20}$ Their analysis is based on Polhamus suction analogy.21 They extended the original work of Pollianius un delta wings to include arbitrary planforms and transonic flow. Their analysis is not repeated here, but their equations are given in the present nomenclature.

Their expression for $C_{N}(\alpha)$ is

$$
F_{N}(\alpha)-K_{p} \sin \alpha \cos \alpha+K_{v} \sin ^{2} \alpha .
$$

Note that this equation shows $C_{N}$ continually increasing with $\alpha$ and, as a result, is not appropriate for angles near or greater than the stall angle. Although Lecat and Reitschlin state their method is applicable to subsonic and supersonic flow, the present analysis is considercd appropriate for subsonic flow and only supersonic flow with subsonic leading edges.

The calculation procedure for $K_{p}$ and $K_{v}$ is as follows:

(1) Calculate $\hat{b}_{0}-\hat{b}, \hat{c}_{r}$ and $\hat{A}_{e}$ from Eqs (31) and (32).

(2) Calculate the distance from $x_{1}$ to the aftmost point of maximum span, $c_{r}^{*}$

$\dot{c}_{r}^{*}=\dot{c}_{r}+\left(\widehat{\left.b_{0}-a\right)} \tan \hat{\Lambda}_{t e}\right.$

(3) Calculate the sweep of the semispan diagonal, $\gamma$.

$\gamma=\tan ^{-1}\left[\dot{\mathrm{c}}_{\mathrm{r}} /\left(\mathrm{b}_{\mathrm{o}}-\mathrm{a}\right)+\tan \hat{\Lambda}_{\mathrm{te}}\right]$

(4) Calculate the ratio of planform area to rectangular reference area, $S_{\mathfrak{f}} / S_{f}^{*}$

$\mathrm{S}_{\mathrm{f}} / \mathrm{S}_{\mathrm{f}}^{*}=2 j\left[\hat{\mathrm{A}}_{\mathrm{e}} \tan \gamma\right]$

(5) If $M_{\infty}>1$, calculate the complement of the Mach angle, $\gamma_{M}$.

$\gamma_{M}=\cos ^{-1}\left(1 / M_{\infty}\right)$ 
(6) Calculate the planform parameter, $\mathrm{p}^{*}$.

$$
p^{\cdot}= \begin{cases}S_{f} / S_{i}^{*} & \text { for } M_{\infty} \leq 1 \\ \frac{S_{f} / S_{i}^{*}}{1-\left[\tan \gamma_{M} /(2 \tan \gamma)\right]} & \text { for } M_{\infty}>1\end{cases}
$$

(7) Calculate the planform angle, $\psi$.

$$
\psi= \begin{cases}\tan ^{-1}\left(2 p^{*} \tan \gamma\right) & M_{\infty} \leq 1 \\ \tan ^{-1}\left[2 p^{*}\left(\tan \gamma-\tan \gamma_{M}\right)\right] & M_{\infty}>1\end{cases}
$$

(8) Calculate the compressibility factor, $\beta$.

$$
B= \begin{cases}\sqrt{1-M_{\infty}^{2}} & M_{\infty} \leq 1 \\ \sqrt{M_{\infty}^{2}-1} & M_{\infty}>1\end{cases}
$$

(9) Calculate the potential flow lift coefficient, $\mathrm{K}_{\mathrm{p}}$.

$$
\mathrm{K}_{\mathrm{p}}=\frac{4 \pi}{\tan \psi+\sqrt{\tan ^{2} \psi+\left(\sin ^{2} \psi / \mathrm{p}^{2}\right)+4 \beta^{2}}} .
$$

(10)

Calculate the vortex flow lift coefficient, $K_{v}$.

$$
K_{v}=\left[K_{p}-\left(K_{p}^{2} \tan \psi\right) / 4 \pi\right] \sqrt{1+\tan ^{2} \psi},
$$

An empirical expression was devised for the normal force coefficient, $\mathrm{C}_{\mathrm{N}}\left(\alpha_{\ell}\right)$, based on the expression of Lecat and Rietschlin, Eq (33). The new expression is more general since it applies both at stall and beyond stall. This expression was constructed after examining and comparing a large number of trial expressions with experimental data ${ }^{10,15-23}$ for a wide variety of planforms. This expression is

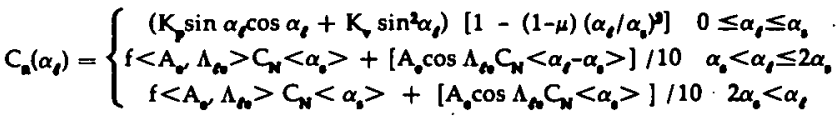

where the symbol $\langle>$ is used to denote functional dependence in situations where the standard symbol, which is a set of parentheses, would be confusing. Also,

$$
\begin{aligned}
\mu= & 0.9-0.2 \mathrm{~A}_{\mathrm{e}} \sin 2 \Lambda_{\ell \mathrm{e}} \\
\alpha_{\mathrm{s}}= & 38\left[1+0.02\left(\mathrm{~A}_{\mathrm{e}}-2\right)^{4}\right]\left[1+1.1\left(\mathrm{~A}_{\mathrm{e}}-1\right)^{2.5} \cos ^{2} \Lambda_{\ell \mathrm{e}}\right] . \\
& \left\{1+\left[6\left(\mathrm{~A}_{\mathrm{e}}-1\right)^{2} \sin ^{4} \Lambda_{\ell \mathrm{e}}\right] / \mathrm{e}^{\mathrm{A}_{\mathrm{e}}}\right\} /\left[\cos \Lambda_{\ell \mathrm{e}}+1\right]^{2\left(\mathrm{~A}_{\mathrm{e}}-1\right)}
\end{aligned}
$$

$$
\begin{aligned}
& f\left(\mathrm{~A}_{\mathrm{e}^{\prime}} \Lambda_{\ell \mathrm{e}}\right)=1-0.35\left\{1+\frac{1}{8} \mathrm{e}^{\mathrm{A}_{\mathrm{e}}} \sin ^{\frac{1}{\mathrm{~A}_{\mathrm{e}}}} \Lambda_{\ell \mathrm{e}}\right. \\
& \left.\therefore \sin \left[\left(\mathrm{A}_{\mathrm{e}}-2.1\right) \Lambda_{\ell \mathrm{e}}\right]\right\} /\left[1+0.5\left(\mathrm{~A}_{\mathrm{e}}-1\right)^{3}\right]
\end{aligned}
$$

and $K_{p}$ and $K_{v}$ are given by Eq (33).

As can be seen, $\mathrm{C}_{\mathrm{N}}\left(\alpha_{\ell}\right)$ is composed of three angle of attack ranges: zero angle up to the stall angle, stall up to twice the stall angle, and angles larger than twice the stall angle. For angles less than $\alpha_{s}$, the expression of Lecat and Reitschlin ${ }^{20}$ (Eq (33)) has been modified for $\alpha_{\ell}$ near $\alpha_{s}$. Equation (35a) for $\alpha_{\ell}<\alpha_{s}$ shows that as $\alpha_{\ell}$ approaches $\alpha_{s}$ the normal force coefficient increases more slowly. This characteristic is consistent with experimental measurements. The parameter $\mu$ represents the portion of $C_{N}$ predicted by Lecat and Reitschlin which remains at $\alpha_{\ell}=\alpha_{s}^{-}$. The expression for $\alpha_{s}$, although rather lengthy, gives a good estimate of the stall angle (in degrees) for planforms with aspect ratios from 1 to 5 and leading edge sweep from $0^{\circ}$ to $70^{\circ}$.

$f\left(A_{e}, \Lambda_{\ell e}\right)$ represents the portion of $C_{N}\left(\alpha_{s}\right)$ existing for $\alpha_{\ell}=\alpha_{\mathrm{s}}^{+}$. Note that $\mu, \alpha_{\mathrm{s}}$, and $\mathrm{f}\left(\mathrm{A}_{\mathrm{e}}, \Lambda_{\ell \mathrm{e}}\right)$ show no dependence on free-stream Mach number. This is not necessarily a reflection of the physics, but simply an admission of lack of data.

$\mathrm{C}_{\mathrm{N}}\left(\alpha_{\ell}\right)$ as predicted by Eq (35) was compared with data for a very wide variety of planform geometries in order to determine its range of applicability. Figures 13 through 22 show typical comparisons of Eq (35) with experimental data for incompressible flow. The figures are placed in order of increasing aspect ratio from 1 to 4 . The leading edge sweep angle varies from $0^{\circ}$ to $70^{\circ}$; planform geometries include: rectangular, diamond, clipped delta, delta, arrow, clipped arrow, and trapezoidal. The comparisons are good for all planforms except that for the aspect ratio 3 trapezoid (Figure 21) and rectangular planforms of $A_{e} \geq 3$ (comparisons not shown). For these types of planforms (i.e., high aspect ratio with small leading edge sweep), the normal force past stall is significantly overestimated by the present expression.

\section{f. Fin - Fin Interference}

Two types of fin - fin interference are included in the present lifting theory. The first type is caused by control deflection of the fins, and the second type by the rolling motion of the missile. When a control deflection is input to a fin, the lifting flow field of that fin induces an angle of attack on the adjacent fins. This induced angle of attack causes the adjacent fins to generate forces and moments which depend 
on the magnitude and direction of the control input. Rolling motion interference occurs for a similar reason except that the angle of attack of a fin is generated by the angular velocity of the fin relative to the oncoming stream. Control deflection interference will be considered first and then rolling motion interference will be analyzed. Results of slenderbody theory are used to evaluate both types of interference considered here.

Figure 23 (taken from Reference 24) shows the interference flow fields and pressures induced on adjacent fins for two types of control input: positive pitch control and positive roll control with horizontal fins. For the pitch control, it is seen that a negative pressure coefficient is produced on both sides of the top fin and a positive pressure coefficient is generated on both sides of the bottom fin. For roll contol (i.e., differential deflection), a negative pressure is generated on the top right and bottom left sides of the vertical fins, and a positive pressure is generated on the top left and bottom right sides of the vertical fins. In the present analysis these induced pressures are included by considering the adjacent fins to be at an effective deflection angle.

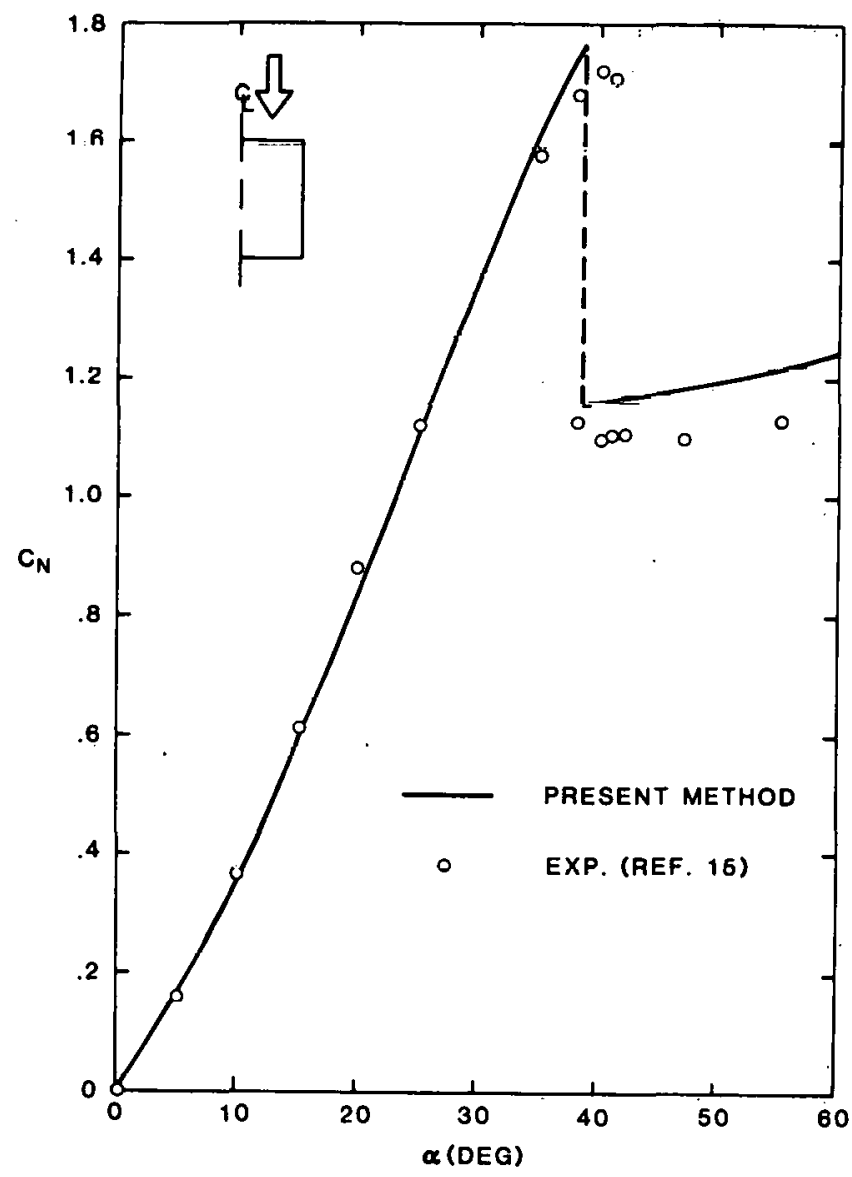

Figure 13. Normal Force Coefficient vs $\alpha$ for Rectangular Planform $\left(A_{e}=1\right)$

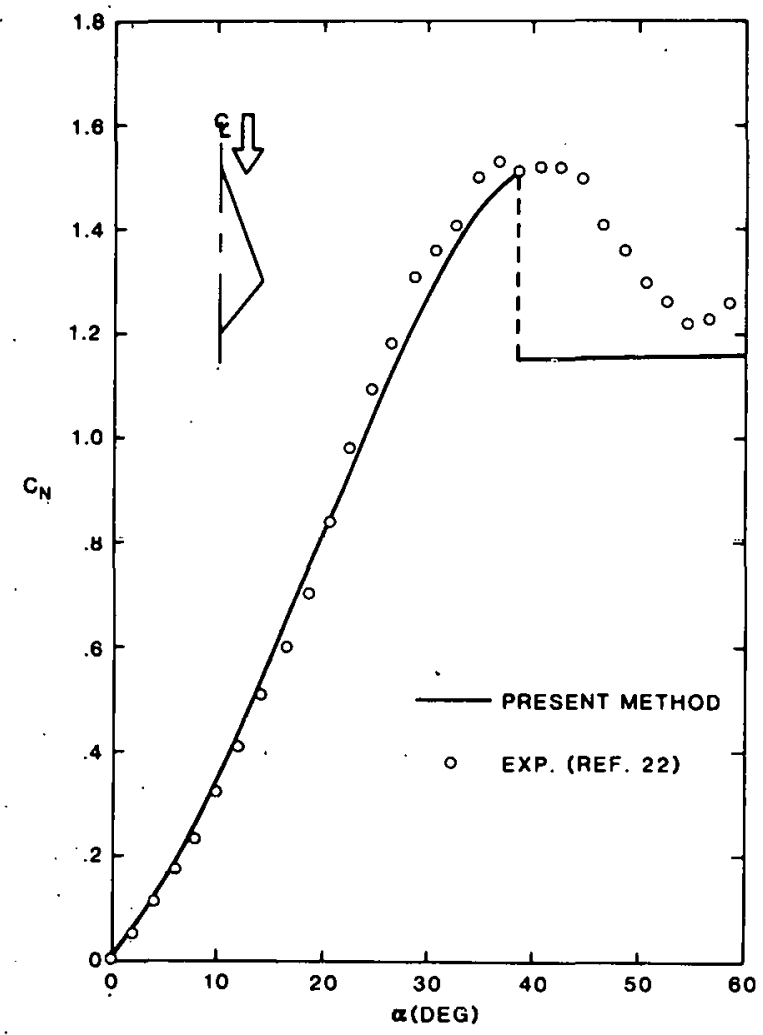

Figure 14. Normal Force Coefficient vs $\alpha$ for Diamond Planform $\left(\Lambda_{\mathrm{e}}=1\right)$

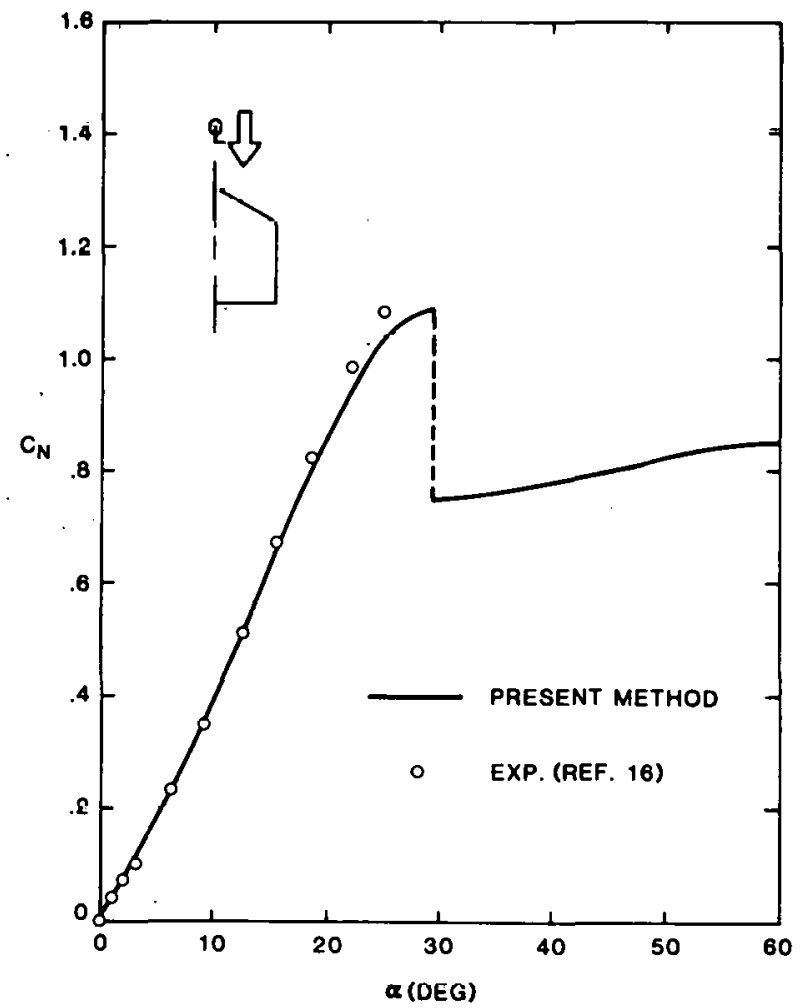

Figure 15. Normal Force Coefficient vs $\alpha$ for Clipped Delta Planform $\left(\mathrm{A}_{\mathrm{e}}=1.23\right)$ 


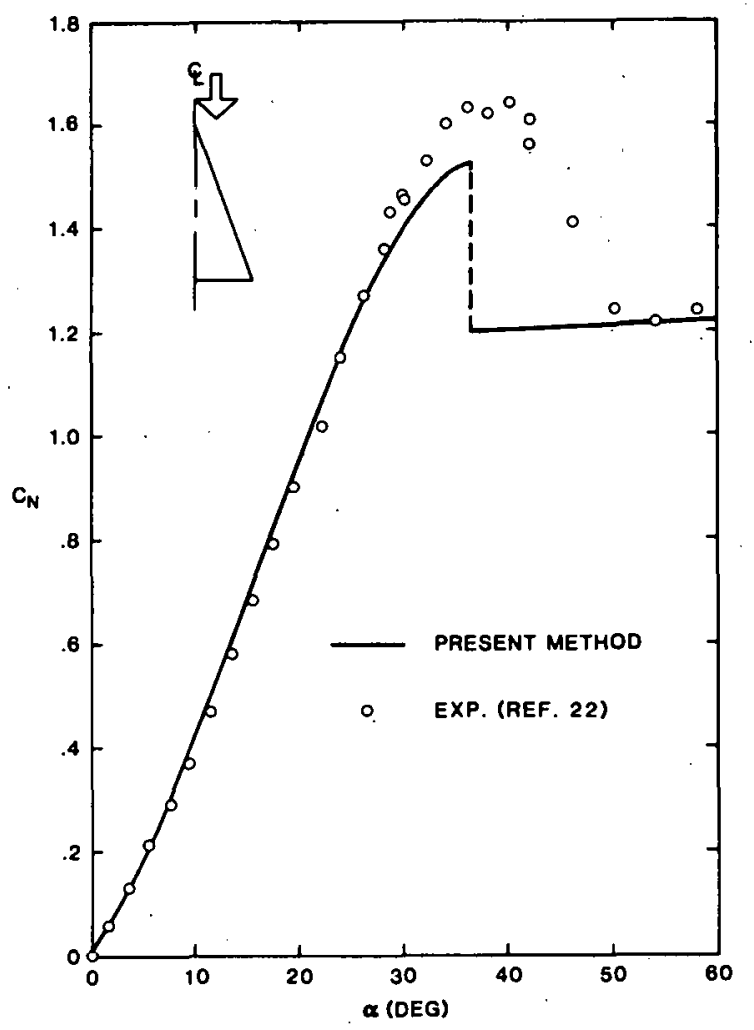

Figure 16. Normal Force Coefficient vs $\alpha$ for Delta Planform $\left(\Lambda_{\mathrm{t}}=1.46\right)$

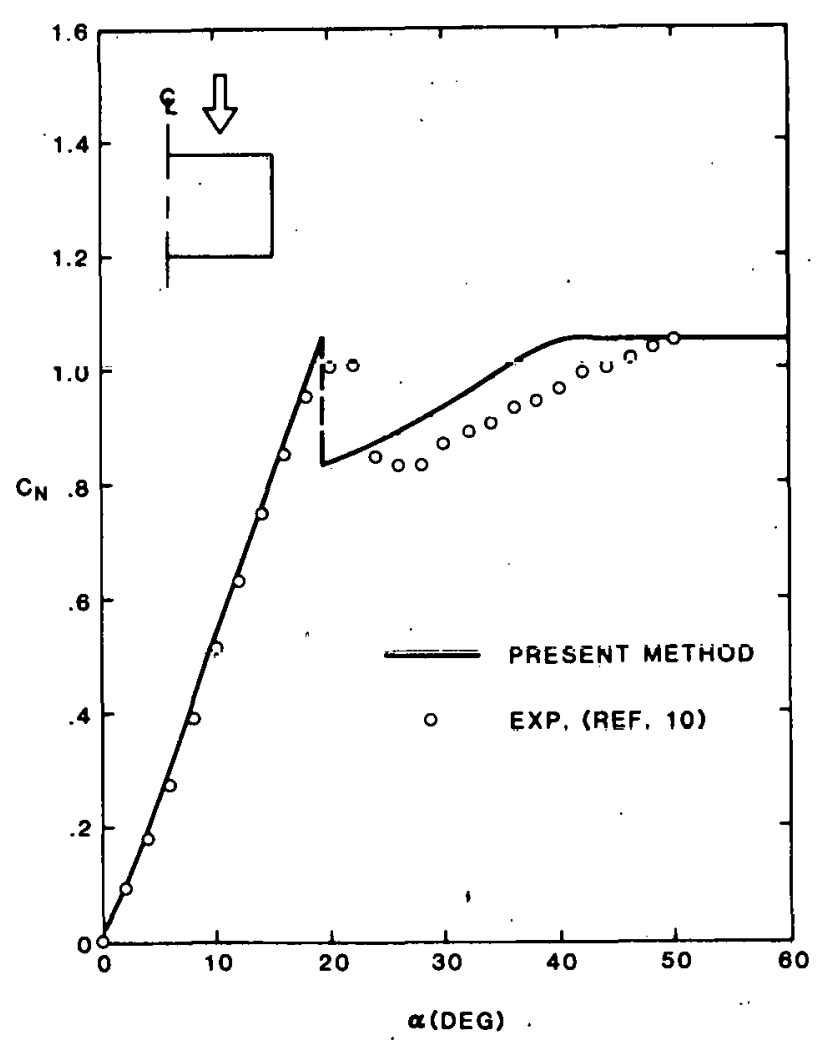

Figure 17. Normal Force Coefficient vs $\alpha$ for Rectangular Planform $\left(A_{e}=2\right)$

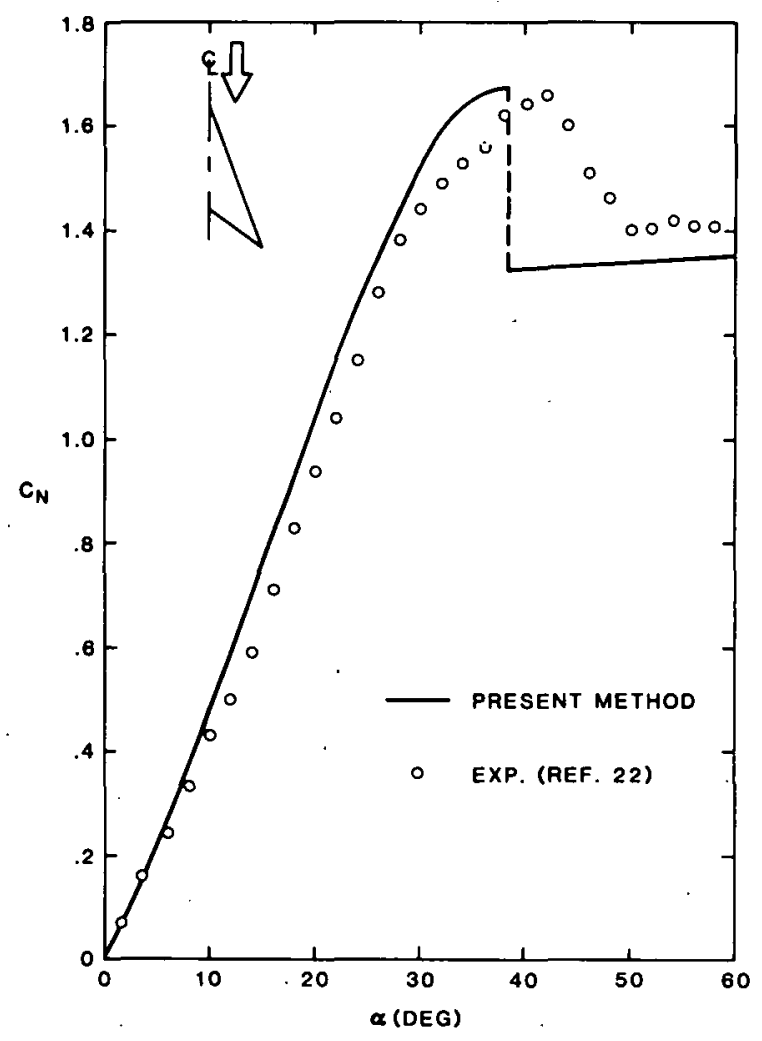

Figure 18. Normal Force Coefficient vs $\alpha$ for Arrow Planform $\left(A_{c}=2\right)$

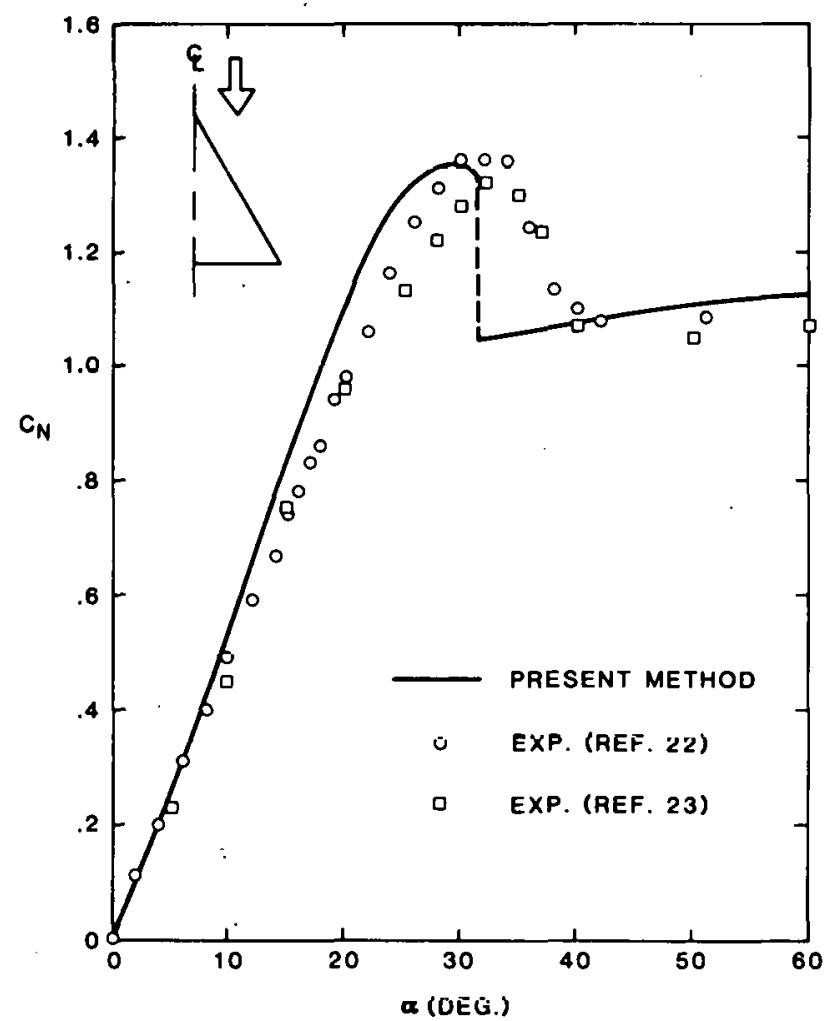

Figure 19. Normal Force Coefficient vs $\alpha$ for Delta Planform $\left(A_{e}=2.31\right)$. 


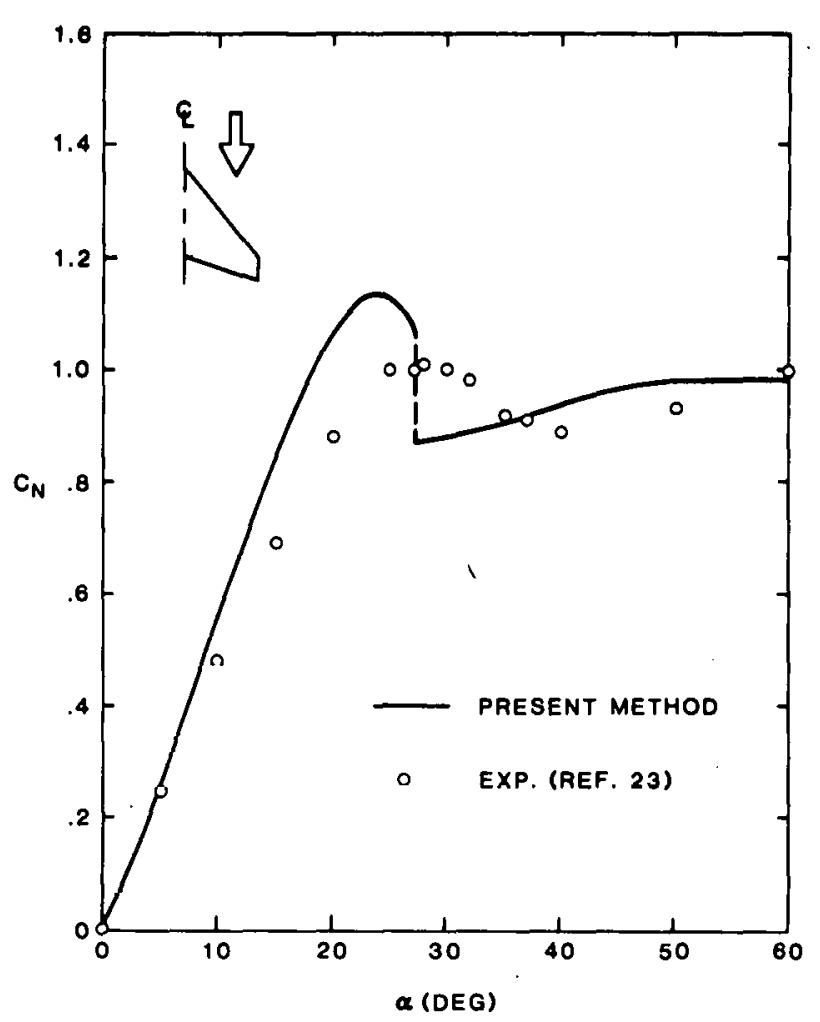

Figure 20. Normal Force Coefficient vs $\alpha$ for Clipped. Arrow Planform $\left(A_{e}=2.61\right)$

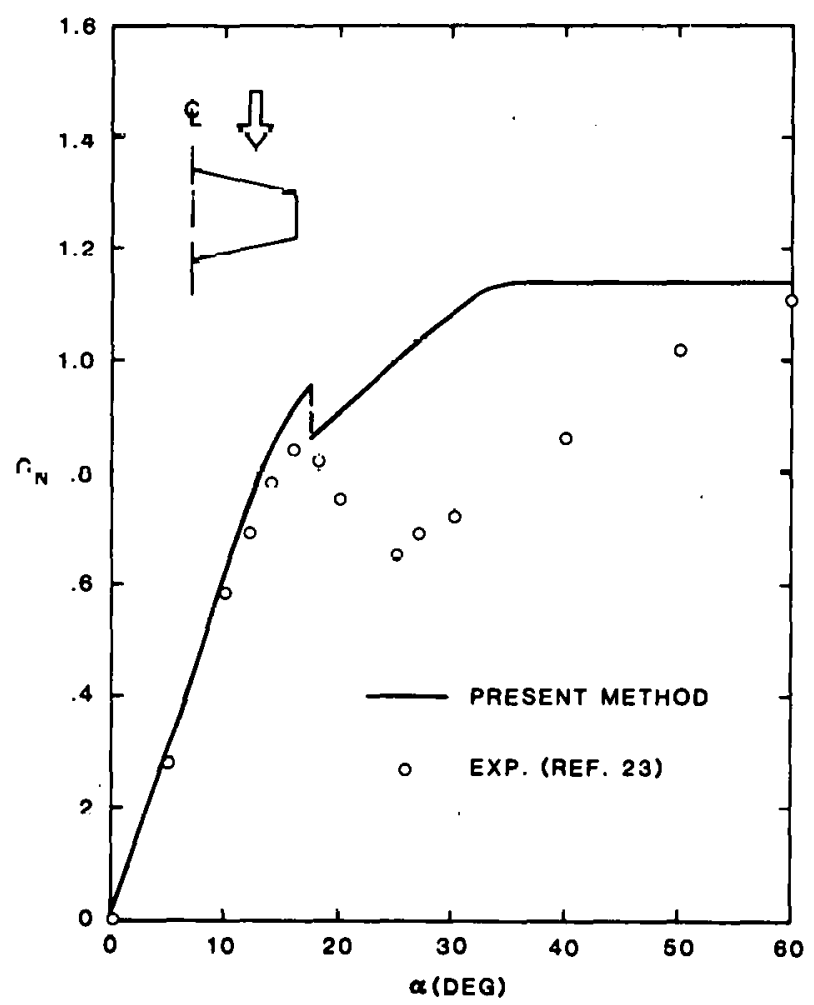

Figure 21. Normal Force Coefficient vs $\alpha$ for Trapezoidal Planform $\left(A_{e}=3\right.$.)

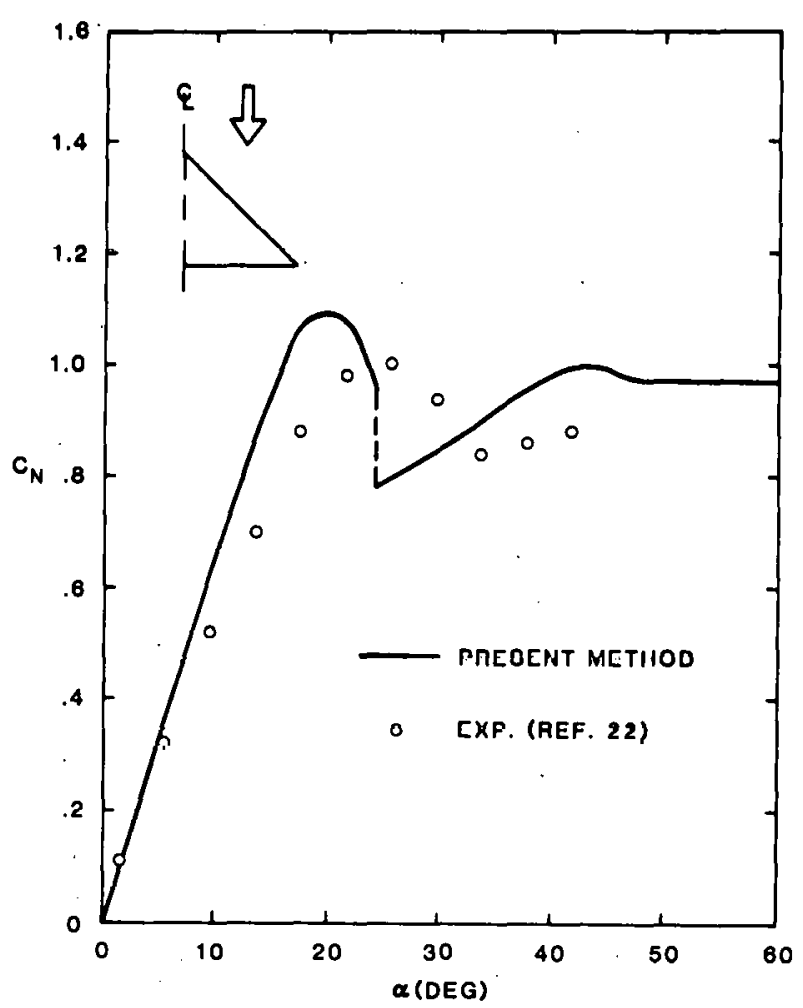

Figure 22. Normal Force Coefficient vs $\alpha$ for Delta Planform $\left(\mathrm{A}_{\mathrm{e}}=4\right.$.)

Induced Flow Field

Induced Pressure field
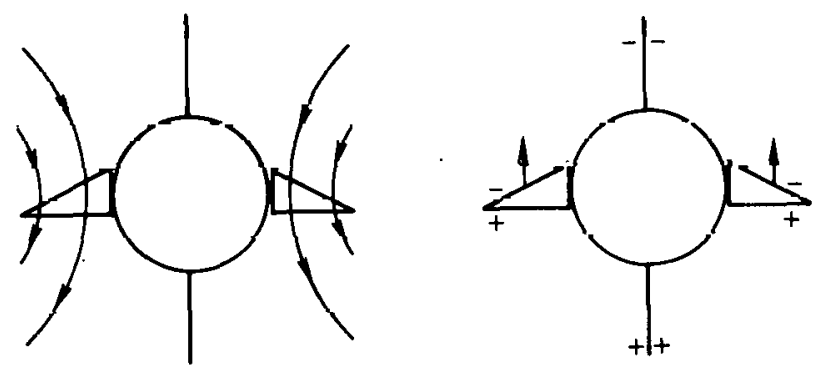

a) Pitch control
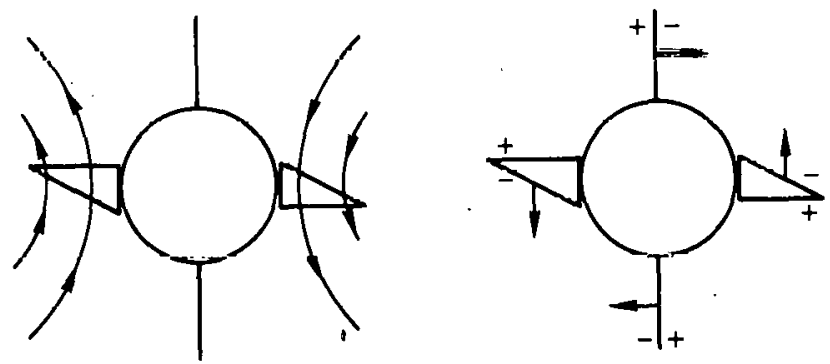

b) Roll Control

Figure 23. Control Interference for Pitch and Roll Control (from Ref. 24) 
Consider the induced pressures on adjacent fins for individual deflection of each fin. Shown in Figure 24 is the induced pressure and effective deflection force for positive deflection of each fin. If we let $I_{d}$ be the deflection interference coefficient, the following equations describe the interference depicted in Figure 24.

$$
\begin{aligned}
& \text { for } \delta_{1}>0\left\{\begin{array}{l}
\delta_{e_{2}}=I_{d} \delta_{1} \\
\delta_{e_{3}}=0 \\
\delta_{e_{4}}=-I_{d} \delta_{1}
\end{array} \quad \text { for } \delta_{2}>0\left\{\begin{array}{l}
\delta_{e_{1}}^{:}=I_{d} \delta_{2} \\
\delta_{e_{3}}=-I_{d} \delta_{2} \\
\delta_{e_{4}}=0
\end{array}\right.\right. \\
& \text { for } \delta_{3}>0\left\{\begin{array}{l}
\delta_{e_{1}}=0 \\
\delta_{e_{2}}=-I_{d} \delta_{3} \\
\delta_{e_{4}}=I_{d} \delta_{3}
\end{array} \quad \text { for } \delta_{4}>0\left\{\begin{array}{l}
\delta_{e_{1}}=-I_{d} \delta_{4} \\
\delta_{e_{2}}=0 \\
\delta_{e_{3}}=I_{d} \delta_{4}
\end{array}\right.\right.
\end{aligned}
$$

Summing all of the effective control deflections given in Eq (36) and including the actual control deflection, one obtains

$$
\begin{aligned}
& \delta_{\mathrm{e}_{1}}=\delta_{1}+\mathrm{I}_{\mathrm{d}}\left(\delta_{2}-\delta_{4}\right) \\
& \delta_{\mathrm{e}_{2}}=\delta_{2}+\mathrm{I}_{\mathrm{d}}\left(\delta_{1}-\delta_{3}\right) \\
& \delta_{\mathrm{e}_{3}}=\delta_{3}+\mathrm{I}_{\mathrm{d}}\left(\delta_{4}-\delta_{2}\right) \\
& \delta_{\mathrm{e}_{4}}=\delta_{4}+\mathrm{I}_{\mathrm{d}}\left(\delta_{3}-\delta_{1}\right)
\end{aligned}
$$

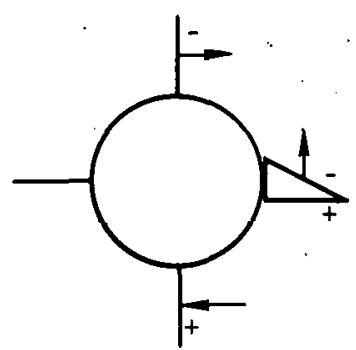

a) Panel 1 Deflection

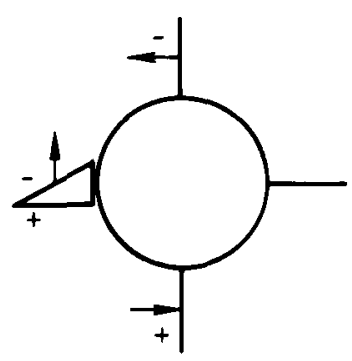

c) Panel 3 Deflection

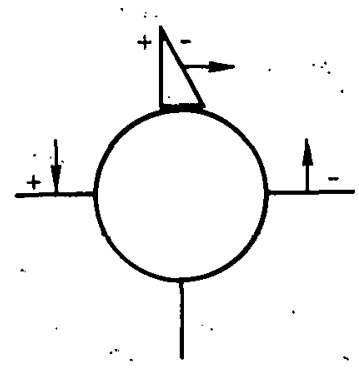

b) Panel 2 Deflection

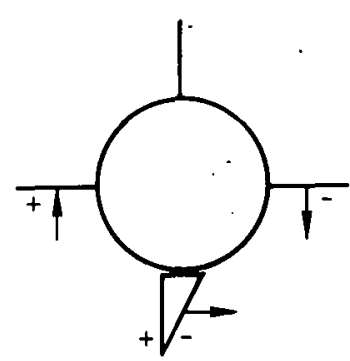

d) Panel Deflection
Figure 24. Control Interference for Individual Panel Deflection
$I_{d}$ is evaluated by using the slender body theory results of Adams and Dugan. ${ }^{25}$ They derived results - from the roll moment coefficient derivative, $C_{\ell b}$, vs $a / b_{0}$ for differential deflection of two fins. They further showed results for the roll moment coefficient induced on the vertical fins because of differential deflection of the horizontal fins. Assuming the induced angle of attack of the upwash and downwash of the deflected fin on the undeflected fin is constant along the span, one can write

$$
\frac{\left[\mathrm{C}_{\ell_{\mathrm{b}}}\right]_{\text {induced }}}{\left[\mathrm{C}_{\ell_{b}}\right]_{\text {deflected }}}=\frac{\delta_{\mathrm{e}}}{\delta}:
$$

This equation can be proven, given the stated assumption, by writing the roll moment of the induced and deflected fin in double integral form. Noting that the ratio shown in Eq (38) is for differential deflection of two fins, one has

$$
\mathrm{I}_{\mathrm{d}}=\frac{1}{2} \frac{\left[\mathrm{C}_{\ell_{b}}\right]_{\text {induced }}}{\left[\mathrm{C}_{\ell_{b}}\right]_{\text {deflected }}}
$$

Figure 25 plots $I_{d}$ vs $a / b_{0}$, using the results of Adams and Dugan for $\left[\mathrm{C}_{\ell_{\delta}}\right]_{\text {induced }}$ and $\left[\mathrm{C}_{\ell_{b}}\right]_{\text {deflected }}$; it should be noted that they use the planform aspect ratio of the fins, $A_{p}$. In the present nomenclature

$$
A_{p}=b^{2} / S_{f_{p}}
$$

where $S_{f_{p}}$ is the planform area of two fins, including their imaginary extension through the body. It can be shown that

$$
S_{f_{p}}=2 S_{f}+a\left[2 c_{r}+a\left(\tan \Lambda_{f e}-\tan \Lambda_{t e}\right)\right] .
$$

$I_{d}$, as given in Figure 25, is used in Eq (37) to calculate the effective control deflection of each fin.

Now consider the case of fin - fin interference due to rolling motion. The strategy of this derivation is to determine the interference coefficient by matching the present roll moment formulation for zero angle of attack of the body to that obtained by slender body theory. Assuming $\alpha_{\mathrm{b}}=0$ and the rolling speed is $\phi$, the differential roll moment can be written (Figure 26)

$$
\mathrm{d} \ell=4 \mathrm{rC}_{\mathrm{n}} \frac{1}{2} \rho_{\infty}\left[\mathrm{U}_{\infty}+(\dot{\phi} \mathrm{r})\right]^{2} \mathrm{~d} x \mathrm{dr}
$$


Assuming the rolling speed is small compared to $U_{\infty}$ and substituting in $\mathrm{C}_{\mathrm{n}}$ from Eq (14), one has

$$
\mathrm{d} \ell=4 \mathrm{q}_{\infty} \mathrm{r} \sigma \mathrm{C}_{\mathrm{n}}\left(\alpha_{\mathrm{e}}\right) \mathrm{C}(\mathrm{x}, \mathrm{r}) \mathrm{S}(\mathrm{r}) \mathrm{dx} \mathrm{dr}
$$

where $\alpha_{\mathrm{e}}$ is the effective local angle of attack along the span. Adams and Dugan ${ }^{25}$ showed that the spanwise loading induced on one fin by the other fins was roughly quadratic along the span. In the present analysis, this induced loading will be simply approximated by a linear spanwise function; therefore,

$$
\alpha_{\mathrm{e}}=-\frac{\dot{\phi r}}{\mathrm{U}_{\omega}}+\mathrm{I}_{\mathrm{P}} \frac{\dot{\phi} \mathrm{r}}{\mathrm{U}_{\omega}}
$$

where $I_{p}$ is the rolling motion interference coefficient. The first term in Eq (41b) is the local angle of attack caused by rolling speed and the second is the reduction in local angle of attack caused by fin - fin interference.

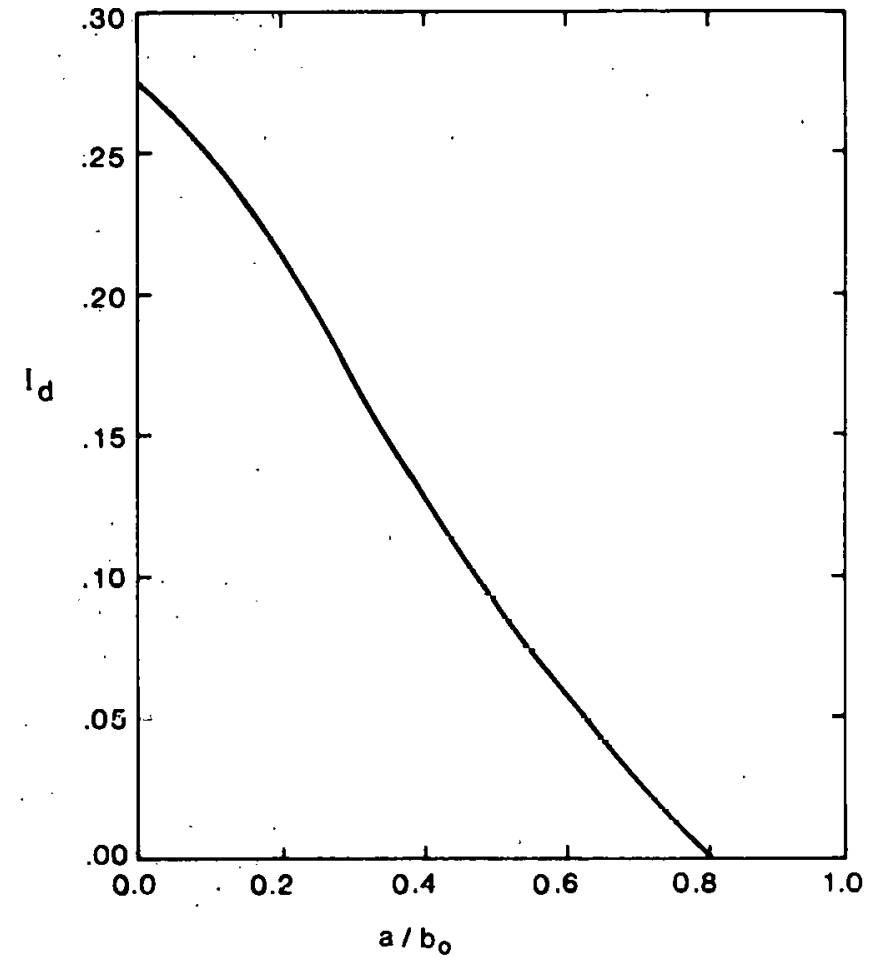

Figure 25. Deflection Interference Coefficient vs $a / b_{0}$

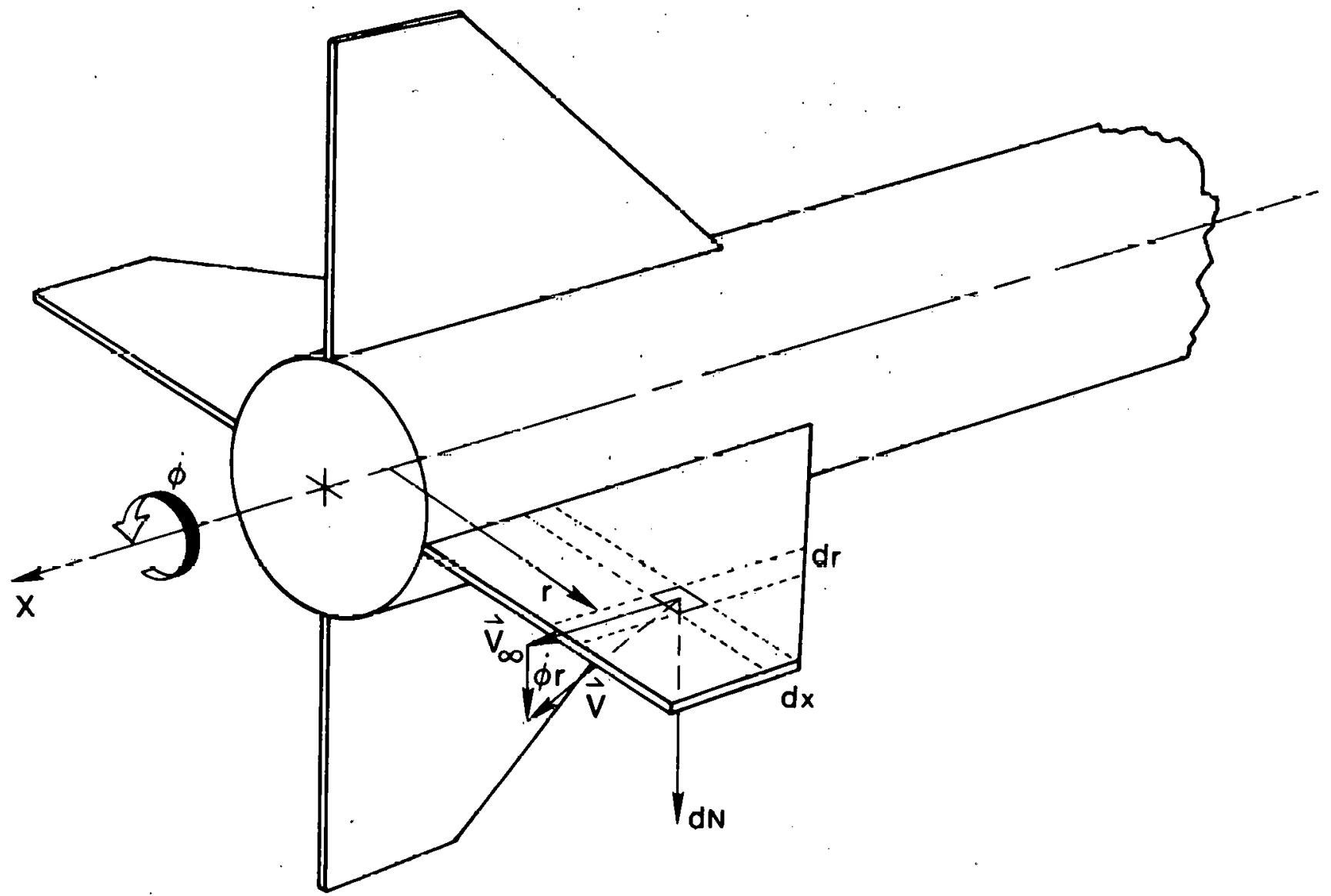

Figure 26. Induced Angle of Attack Due to Rolling Speed 
Integrating over the surface of the fin, Eq (41a) becomes

$$
\ell=4 \sigma \mathrm{q}_{\infty} \int_{\mathrm{a}}^{\mathrm{b}_{0}} \int_{\mathrm{x}_{\ell \mathrm{e}}}^{\mathrm{x}_{\mathrm{te}}} \mathrm{r} \mathrm{C}_{\mathrm{N}}\left(\alpha_{\mathrm{e}}\right) \mathrm{C}(\mathrm{x}, \mathrm{r}) \mathrm{S}(\mathrm{r}) \mathrm{dr} \mathrm{dx}
$$

Assuming $\alpha_{\mathrm{e}}$ is small and using Eq (41b), Eq (42) can be written

$$
\ell=-4 \sigma \mathrm{q}_{\infty} \mathrm{C}_{\mathrm{N}_{\alpha}}\left(1-\mathrm{I}_{\mathrm{p}}\right) \frac{\dot{\phi}}{\mathrm{U}_{\infty}} \int_{\mathrm{a}}^{\mathrm{b}_{\mathrm{o}}} \int_{\mathrm{x}_{\ell \mathrm{e}}}^{\mathrm{x}_{\mathrm{te}}} \mathrm{r}^{2} \mathrm{C}(\mathrm{x}, \mathrm{r}) \mathrm{S}(\mathrm{r}) \mathrm{d} \mathrm{d} \mathrm{d} \mathbf{r} .
$$

Transforming coordinates $(x, r)$ to fin coordinates $(\xi, \eta)$ and nondimensionalizing with respect to $q_{\infty} S_{b} d$, one obtains

$$
\begin{aligned}
C_{\ell}= & -\frac{2}{\pi} \sigma C_{N_{o}} \frac{\left(b_{0}-a\right)}{a^{3} b_{0}}\left(1-I_{p}\right) \frac{\dot{\phi} b_{o}}{U_{\infty}} \cdot \\
& \int_{0}^{1} \int_{0}^{1} \Re \Re^{2}(\eta) \Omega(\eta) C(\xi) S(\eta) d \xi d \eta
\end{aligned}
$$

where

$$
\Re(\eta)=a+\left(b_{0}-a\right) \eta
$$

Taking the partial derivative of $\mathrm{Eq}$ (43) with respect to $p$, where $p=\dot{\phi} b_{0} / U_{\infty}$, and solving for $I_{p}$, one finally obtains

$$
\mathrm{I}_{\mathrm{p}}=1+\frac{\mathrm{C}_{\epsilon_{\mathrm{p}}}}{\frac{2}{\pi} \sigma \frac{\left(\mathrm{b}_{\mathrm{o}}-\mathrm{a}\right)}{\mathrm{a}^{3} \mathrm{~b}_{\mathrm{o}}} \mathrm{C}_{\mathrm{N}_{a}} \int_{0}^{1} \int_{0}^{1} \Re^{2}(\eta) \Omega(\eta) \mathrm{C}(\xi) \mathrm{S}(\eta) \mathrm{d} \xi \mathrm{d} \eta} .
$$

$C_{\ell p}$ is computed from the results of Adams and Dugan (see Figure 27). $C_{N_{\alpha}}$ is obtained by numerically differencing Eq (35) for $\alpha_{\ell} \rightarrow 0$.

\section{Roll Moment}

The above described body flow field and lifting theory will now be applied to the prediction of the roll moment produced by cruciform fins. If one desired, the present analysis could be applied in a straightforward manner to two or three fin configurations. Consider the missile at angle of attack $\alpha_{b}$, roll angle $\phi$, and roll rate $\dot{\phi}$. The roll moment produced by a differential surface element on each of the four fins is

$$
\mathrm{d} \ell=\mathrm{q}_{\infty} \mathrm{r} \sum_{j=1}^{4} \mathrm{C}_{\mathrm{n}_{\mathrm{j}}}\left(\mathrm{U}_{\mathrm{j}} / \mathrm{U}_{\infty}\right)^{2} \mathrm{dx} \mathrm{dr}
$$

where $j$ refers to the $j$ 'th fin and $U_{j}$ is the magnitude of the velocity on the $j^{\prime}$ th fin. Substituting $C_{n}$ from $E q$ (14) and integrating over the fin surfaces, one has

$$
\ell=\sigma q_{\infty} \int_{i}^{b_{0}} \int_{x_{\ell e}}^{x_{\text {te }}} r C(x, r) S(r) \sum_{j=1}^{4} C_{N_{i}}\left(U_{j} / U_{\infty}\right)^{2} d x d r .
$$

Transforming to fin coordinates $(\xi, \eta)$ and nondimensionalizing by $\mathrm{q}_{\infty} \mathrm{s}_{\mathrm{b}} \mathrm{d}$, one obtains the roll moment coefficient

$$
\begin{aligned}
C_{\ell}= & \frac{\sigma\left(b_{0}-a\right)}{2 \pi a^{3}} \int_{0}^{1} \int_{0}^{1} \Re(\eta) \Omega(\eta) C(\xi) S(\eta) . \\
& \sum_{j=1}^{4} C_{N_{i}}\left(U_{j} / U_{\infty}\right)^{2} d \xi d \eta
\end{aligned}
$$

where $\Re(\eta)$ is given by Eq $(43 \mathrm{~b}), \Omega(\eta)$ is given by Eq $(20 b), C(\xi)$ is given by $\mathrm{Eq}(19 \mathrm{a})$, and $S(\eta)$ is given by $\mathrm{Eq}$ (19b).

$\mathrm{U}_{\mathrm{j}}$ is found by taking the magnitude of the local velocity given in Eq (23):

$U_{j}=\sqrt{U_{\infty}^{2} \cos ^{2} \alpha_{b}+\left(v_{q}+\dot{\phi} r \sin \phi\right)^{2}+\left(w_{q}-\dot{\phi} r \cos \phi\right)^{2}}$.

Note that if $\phi$ appears without the subscript $j$ (as in the above equation), the angle $\phi$ is measured from the $y$ axis for the particular fin under consideration.

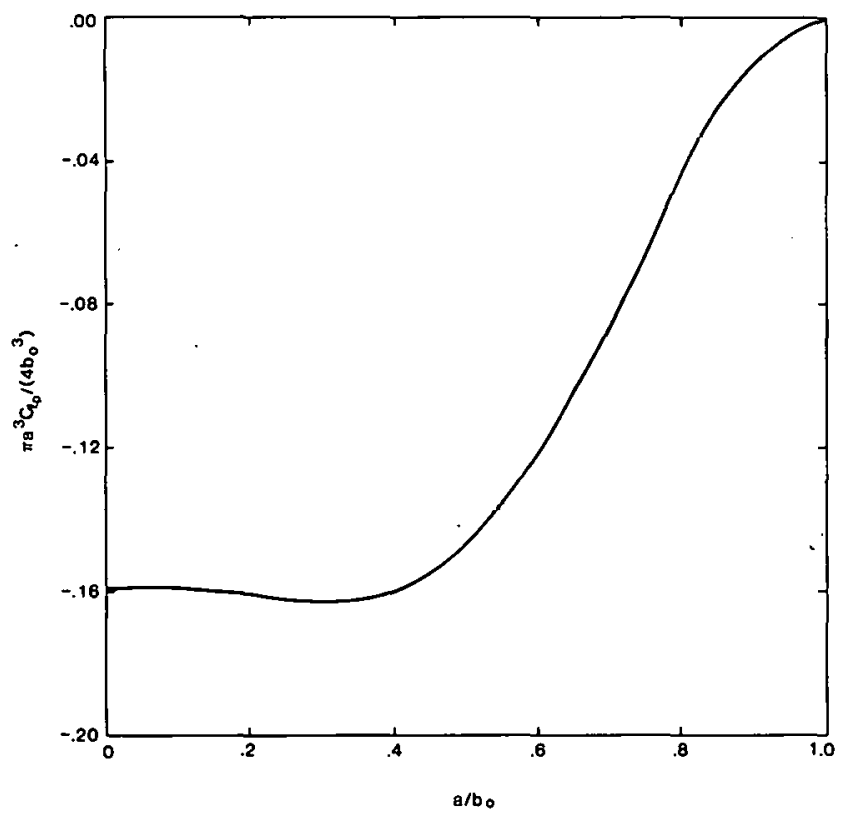

Figure 27. Roll Damping Moment Coefficient vs $a / b_{0}$ 
$C_{N_{1}}$ depends upon the local effective angle of attack $\alpha_{e_{j}}$.To determine this angle, the effective surface normal vector must be found taking into account control deflection and rolling motion interference. Using $\vec{n}$ from $\mathrm{Eq}(22)$, the effective fin deflection angle $\delta_{e_{j}}$ from Eq (37), and noting that the rolling motion interference affects the $\vec{i}$ component of the normal vector, one can write*

$$
\begin{aligned}
\vec{n}_{e_{j}}= & {\left[\sin \left(\Delta \delta_{e_{j}}\right)+I_{p} \dot{\phi} r / U_{\infty}\right] \vec{i} } \\
& -\cos \left(\delta_{e_{j}}\right) \sin \phi \vec{j}+\cos \left(\delta_{e_{j}}\right) \cos \phi \vec{k} .
\end{aligned}
$$

Substituting $\overrightarrow{\mathrm{n}}_{\mathrm{e}}$ and $\overrightarrow{\mathrm{V}}$ into the equation for $\alpha_{\ell}$, Eq (21), one obtains the local effective angle of attack.

$$
\begin{aligned}
\alpha_{e_{i}} & =\sin ^{-1}\left[\left\{U_{\infty} \cos \alpha_{b}\left[\sin \left(\Delta \delta_{e_{i}}\right)+I_{p} \dot{\phi} r / U_{m}\right]\right.\right. \\
& -\left(v_{c_{j}}+\dot{\phi} \mathrm{r} \sin \phi\right) \cos \left(\delta_{e_{j}}\right) \sin \phi \\
& \left.\left.+\left(w_{c_{i}}-\dot{\phi} r \cos \phi\right) \cos \left(\delta_{e_{j}}\right) \cos \phi\right\} /\left(\left|\vec{n}_{e_{j}}\right| U_{j}\right)\right]
\end{aligned}
$$

where

$$
\left|\vec{n}_{\mathbf{e}_{j}}\right|=1+2 \sin \left(\Delta \delta_{e_{j}}\right) I_{p} \dot{\phi} r / U_{\infty}+\left(I_{p} \dot{\phi} r / U_{\infty}\right)^{2} .
$$

\section{Normal Force and Pitch Moment}

The equations for the normal force and pitch moment produced by the fins will now be considered. The difterential fin force in the $z$ direction produced by the four fins is (see Figure 28 )**

$$
\begin{aligned}
\mathrm{dF}_{\mathrm{z}} & =\mathrm{dN}_{1} \cos \delta_{1} \cos \phi_{1}-\mathrm{dN}_{2} \cos \delta_{2} \sin \delta_{2} \\
& -\mathrm{dN}_{3} \cos \delta_{3} \cos \phi_{3}+\mathrm{dN}_{4} \cos \delta_{4} \sin \phi_{4} .
\end{aligned}
$$

Writing the right side of this equation as a summation and substituting $\mathrm{dN}_{\mathrm{j}}$ from $\mathrm{Eq}$ (13), one obtains

$\mathrm{dF}_{\mathrm{z}}=\mathrm{q}_{\infty} \sum_{j=1}^{4} \mathrm{C}_{n_{j}}\left(\mathrm{U}_{\mathrm{j}} / \mathrm{U}_{\infty}\right)^{2} \cos \delta_{j} \cos \phi \mathrm{dxdr}$

\footnotetext{
* Reference 10 accounted for rolling motion interference by way of $\vec{i}$ and $\vec{k}$ components of the velocity. That approach is not considered correct because it also changes the magnitude of the velocity. In fin - fin interference the magnitude of the approach velocity to the fin is not changed, but only the angle with which it approaches the fin.

* Note that the sign of $\mathrm{dN}_{\mathrm{j}}$ is determined by its angular relationship to the surface normal vector; that is, positive $d N_{j}$ is in the counterclockwise sense.
}

Substituting in $\mathrm{C}_{\mathrm{n}}$ from $\mathrm{Eq}$ (14) and integrating over the surface of each fin, one has

$$
\begin{aligned}
F_{z}= & \sigma q_{\infty} \int_{a}^{b_{0}} \int_{x_{l e}}^{x_{t e}} C(x, r) S(r) \cdot \\
& \sum_{j=1}^{4} C_{N_{j}}\left(U_{j} / U_{\infty}\right)^{2} \cos \delta_{j} \cos \phi d x d r .
\end{aligned}
$$

Transforming to fin coordinates $(\xi, \eta)$ and nondimensionalizing by $q_{\infty} S_{b}$, one obtains the normal force coefficient due to the fins:

$$
\begin{aligned}
C_{\mathrm{z}}= & \frac{\sigma\left(\mathrm{b}_{0} \ddot{-}-\mathrm{a}\right)}{\pi \mathrm{a}^{2}} \int_{0}^{1} \int_{0}^{1} \Omega(\eta) \mathrm{C}(\xi) \mathrm{S}(\eta) . \\
& \sum_{j=1}^{4} \mathrm{C}_{\mathrm{N}_{\mathrm{j}}}\left(U_{j} / U_{\omega}\right)^{2} \cos \delta_{j} \cos \phi d \xi d \eta
\end{aligned}
$$

The pitch moment produced by the fins is composed of two types of terms: the first term is caused by the normal force just derived and the second by the $\sin \delta_{j}$ component of the normal force on the fins (Figure 28). The second term produces a pure moment (i.e., a couple) on the missile body and is usually much smaller in magnitude than the first term. The differential pitch moment due to both terms from all four fins is

$$
\begin{aligned}
\mathrm{d} m= & -x \mathrm{dN}_{1} \cos \delta_{1} \cos \phi_{1}+\mathrm{rdN_{1 }} \sin \left(\Delta \delta_{1}\right) \sin \phi_{1} \\
& +x \mathrm{dN}_{2} \operatorname{rns} \delta_{2} \sin \phi_{2}+\mathrm{rdN_{2 }} \sin \left(\Delta \delta_{2}\right) \operatorname{sos} \phi_{2} \\
& +x \mathrm{dN}_{3} \cos \delta_{3} \cos \phi_{3}-\mathrm{rdN} \mathrm{rd}_{3} \sin \left(\Delta \delta_{3}\right) \sin \psi_{3} \\
& -x \mathrm{dN}_{4} \cos \delta_{4} \sin \phi_{4}-\mathrm{rdN}_{4} \sin \left(\Delta \delta_{4}\right) \cos \phi_{4}
\end{aligned}
$$

Writing the right side of this equation as a summation and substituting $d N_{j}$ from Eq (13), one obtains

$d i i i=-q_{\infty} \sum_{i=1}^{4} C_{\eta_{i}}\left(U_{i} / U_{\infty}\right)^{2}\left[x \cos \delta_{i} \cos \phi-r \sin \left(\Delta \delta_{j}\right) \sin \phi\right] d x d r$.

Substituting in $\mathrm{C}_{\mathrm{n}}$ from Eq (14) and integrating over the surface of each fin, one has

$$
\begin{aligned}
W= & -\sigma \mathrm{q}_{\infty} \int_{\mathrm{a}}^{\mathrm{b}_{0}} \int_{\mathrm{x}_{\mathrm{ee}}}^{\mathrm{x}_{\mathrm{te}}} \mathrm{C}(\mathrm{x}, \mathrm{r}) \mathrm{S}(\mathrm{r}) \sum_{\mathrm{j}=1}^{4} \mathrm{C}_{\mathrm{N}_{\mathrm{j}}}\left(\mathrm{U}_{\mathrm{i}} / \mathrm{U}_{\infty}\right)^{2} . \\
& {\left[\mathrm{x} \cos \delta_{\mathrm{j}} \cos \phi-\mathrm{r} \sin \left(\Delta \delta_{\mathrm{j}}\right) \sin \phi\right] \mathrm{d} \mathrm{d} \mathrm{dr} . }
\end{aligned}
$$


Transforming to fin coordinates $(\xi, \eta)$ and nondimensionalizing by $q_{\infty} S_{b} d$, one obtains the pitch moment coefficient due to the fins:

$C_{m}=\frac{-\sigma\left(b_{0}-a\right)}{2 \pi a^{3}} \int_{0}^{1} \int_{0}^{1} \Omega(\eta) C(\xi) S(\eta) \sum_{j=1}^{4} C_{N_{j}}\left(U_{j} / U_{\infty}\right)^{2}$.

$\left[\left\{\mathcal{X}^{\prime}(\xi, \eta) \cos \delta_{\mathrm{j}} \cos \phi-\Re(\eta) \sin \left(\Delta \delta_{\mathrm{j}}\right) \sin \phi\right] \mathrm{d} \xi \mathrm{d} \eta\right.$

where

$\mathfrak{t}(\xi, \eta)=x_{1}+c_{r}+\left(b_{0}-a\right) \tan \Lambda_{t e} \eta-\Omega(\eta) \xi$.

\section{Side Force and Yaw Moment}

The derivation of the side force and yaw moment is very similar to that of the normal force and pitch moment and, consequently, is not given. The side force coefficient and the yaw moment coefficient are given by:

$$
\begin{aligned}
C_{y}= & \frac{-\sigma\left(b_{o}-a\right)}{\pi a^{2}} \int_{0}^{1} \int_{0}^{1} \Omega(\eta) C(\xi) S(\eta) . \\
& \sum_{j=1}^{4} C_{N_{i}}\left(U_{j} / U_{\infty}\right)^{2} \cos \delta_{j} \sin \phi d \xi d \eta
\end{aligned}
$$

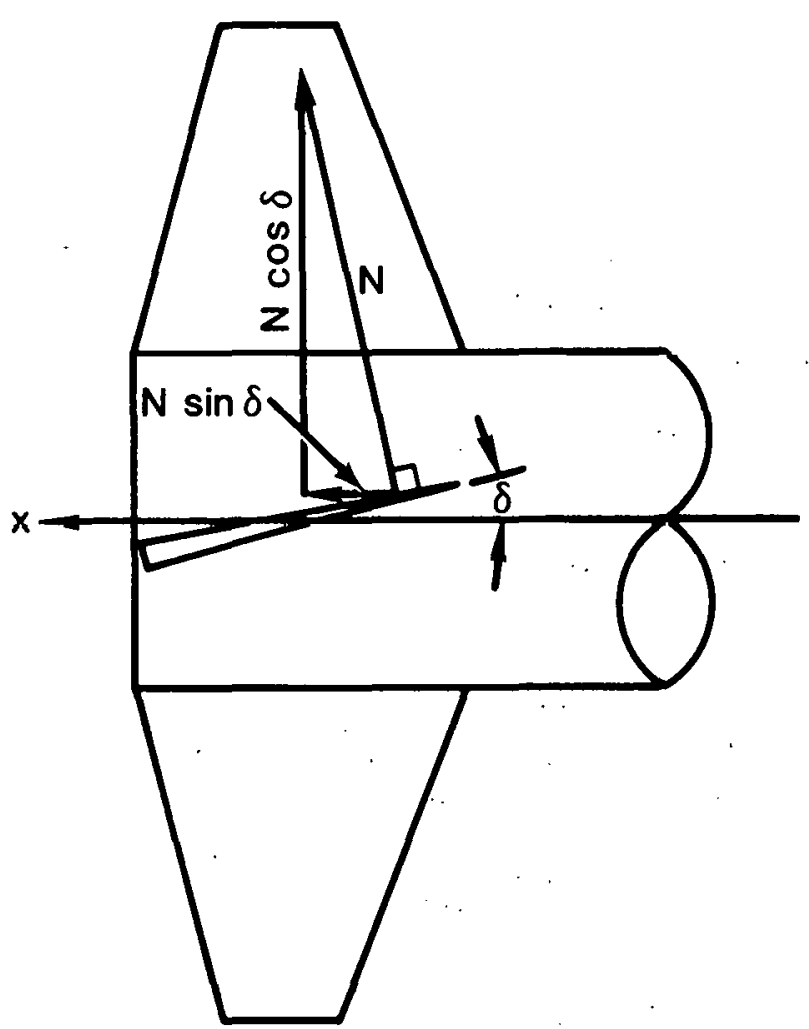

Figure 28. Components of the Fin Normal Force and

$$
\begin{aligned}
\dot{C}_{n}= & \frac{-\sigma\left(\mathrm{b}_{0}-\mathrm{a}\right)}{2 \pi \mathrm{a}^{3}} \int_{0}^{1} \int_{0}^{1} \Omega(\eta) \sum_{\mathrm{i}=1}^{4} \mathrm{C}_{\mathrm{N}_{\mathrm{j}}}\left(\mathrm{U}_{\mathrm{j}} / \mathrm{U}_{\infty}\right)^{2}[X(\xi, \eta) . \\
& \left.\cos \delta_{\mathrm{j}} \sin \phi+\Re(\eta) \sin \left(\Delta \delta_{\mathrm{j}}\right) \cos \phi\right] \mathrm{d} \xi \mathrm{d} \eta .
\end{aligned}
$$

\section{Panel Loads}

The panel loads on the fin surfaces are essentially the same forces and moments derived previously (Roll Moment, Normal Force and Pitch Moment, and Side Force and Yaw Moment) except that they are oriented in a fin coordinate system. Figure 29 shows the coordinate system and sign convention for the panel normal force $(\mathrm{N})$, the panel hinge moment $(\mathrm{H})$, and the panel root bending moment (B). The calculation of panel loads is useful for two reasons: first, they provide the most physically meaningful force and moments with which to compare theoretical predictions and experiment because they do not contain the geometry components of the roll angle and the fin-deflection angle. Second, they directly provide the loads needed by the structural designer and the actuator torques needed by the guidance and control designer.

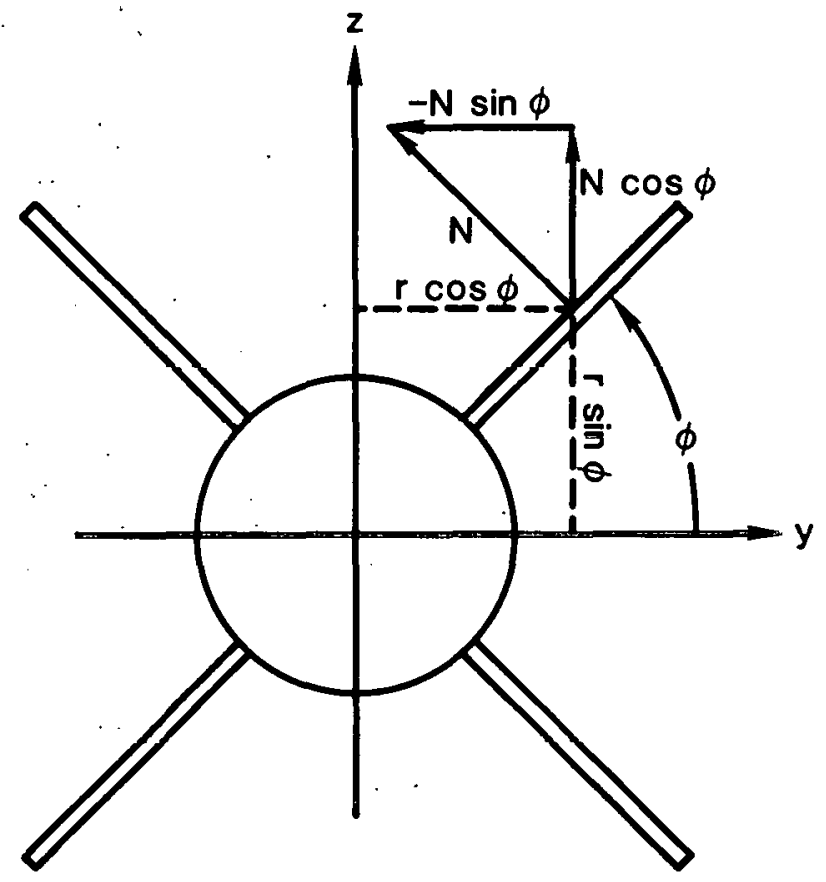




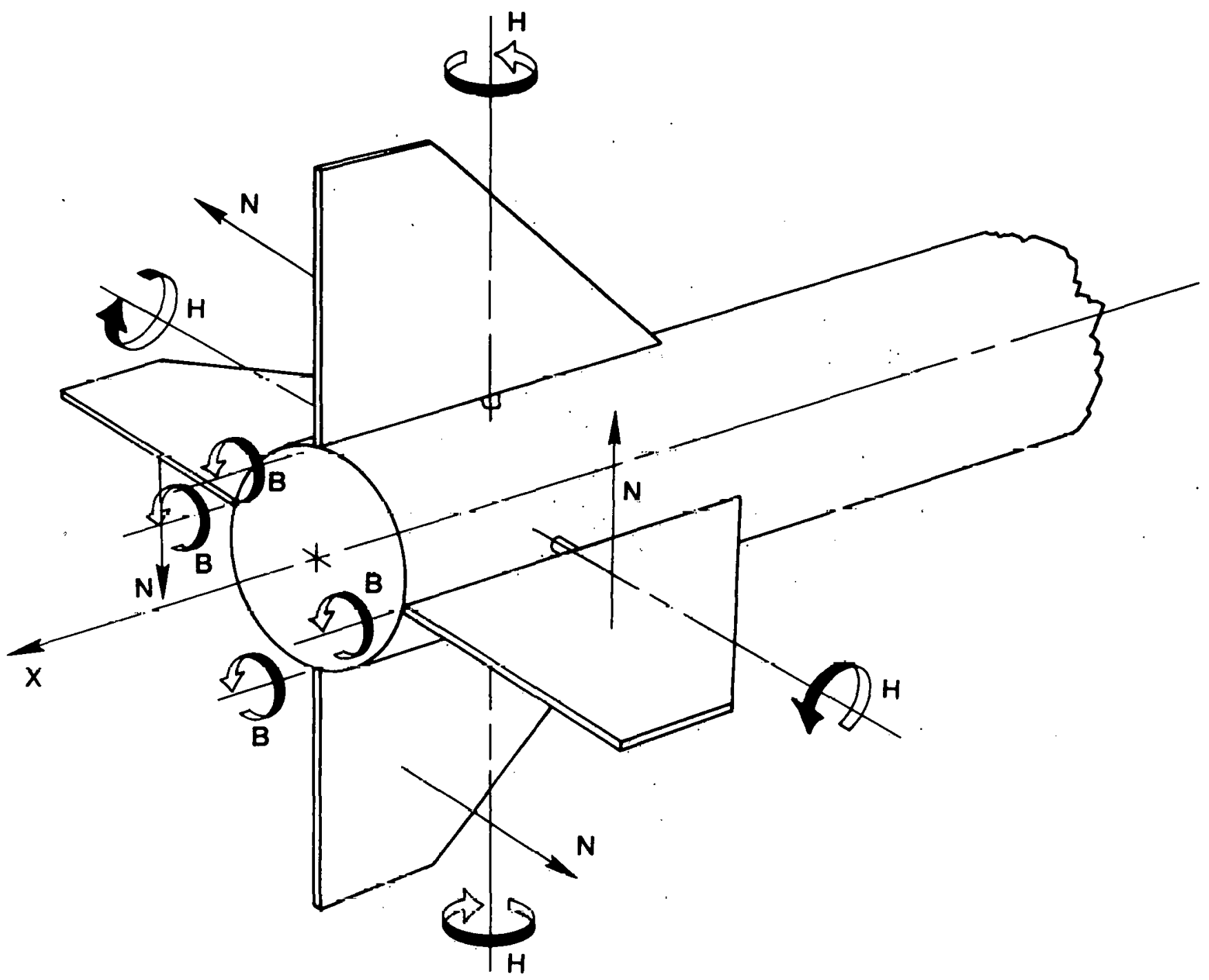

Figure 29. Sign Convention for Panel Normal Force, Hinge Moment, and Root Bending Moment

As the derivation of the panel loads is very similar to the previous derivations, it will only be sketched very briefly. Using Eq (13), the normal force on a fin panel caused by a differential surface element is

$$
\mathrm{dN}=\mathrm{q}_{\infty} \mathrm{C}_{\mathrm{n}}\left(\mathrm{U} / \mathrm{U}_{\infty}\right)^{2} \mathrm{dx} \mathrm{dr} .
$$

Proceeding as before, one obtains the panel normal force coefficient, $N / \mathrm{q}_{\infty} \mathrm{S}_{\mathrm{f}}$ :

$$
\mathrm{C}_{\mathrm{p}}=\frac{\sigma\left(\mathrm{b}_{\mathrm{o}}-\mathrm{a}\right)}{\mathrm{S}_{\mathrm{f}}} \int_{0}^{1} \int_{0}^{1} \Omega(\eta) \mathrm{C}(\xi) \mathrm{S}(\eta) \mathrm{C}_{\mathrm{N}}\left(\mathrm{U} / \mathrm{U}_{\infty}\right)^{2} \mathrm{~d} \xi \mathrm{d} \eta
$$

Recall that positive normal force points in the direction of the counterclockwise rotational sense.
The panel hinge moment due to a differential surface element is

$$
d H=-q_{\infty}\left(x-x_{h}\right) C_{n}\left(U / U_{\infty}\right)^{2} d x d r
$$

where $x_{h}$ is the axial location of the reference line for the hinge moment. Proceeding as before, one obtains the panel hinge moment rnefficient, $\mathrm{H} / \mathrm{q}_{\dot{\infty}} \mathrm{c}_{\mathrm{r}} \mathrm{S}_{\mathrm{l}}$ :

$$
\begin{aligned}
C_{h}= & \frac{-\sigma\left(b_{0}-a\right)}{c_{r} S_{f}} \int_{0}^{1} \int_{0}^{1}\left[\mathfrak{x}(\xi, \eta)-x_{h}\right] . \\
& \Omega(\eta) C(\xi) S(\eta) C_{N}\left(U / U_{\infty}\right)^{2} d \xi d \eta .
\end{aligned}
$$


The panel root bending moment due to a differential surface element is

$$
d B=q_{\infty}(r-a) C_{n}\left(U / U_{\infty}\right)^{2} d x d r .
$$

Proceeding as before, one obtains the panel root bending moment coefficient, $B / q_{\infty}\left(b_{o}-a\right) S_{f}$ :

$$
\begin{gathered}
\mathrm{C}_{\mathrm{b}}=\frac{\sigma\left(\mathrm{b}_{\mathrm{o}}-\mathrm{a}\right)}{\mathrm{S}_{\mathrm{f}}} \int_{0}^{1} \int_{0}^{1} \eta \Omega(\eta) \mathrm{C}(\xi) \mathrm{S}(\eta) . \\
\mathrm{C}_{\mathrm{N}}\left(\mathrm{U} / \mathrm{U}_{\infty}\right)^{2} \mathrm{~d} \xi \mathrm{d} \eta .
\end{gathered}
$$

\section{Results and Discussion}

The results computed and presented in this investigation were dictated solely by the requirement to validate and determine the bounds of the present theory. Consequently, no results are given without experimental measurements. The theory is evaluated by comparing predictions and measurements for six different fin planforms. The detailed geometry and reference from which the data were taken is given in Table 1. The planforms are shown in Figure 30. It can be seen that the aspect ratios range from 1.0 to 2.0 and the leading edge sweep angles range from $0^{\circ}$ to $67.4^{\circ}$.

The results and comparison with experiment are discussed in three sections: panel loads, roll moments, and control forces and moments. The panel loads presented are panel normal force, panel bending moment, and panel hinge moment. Normal force, pitch moment, side force, and yaw moment predictions could have been compared with experimental measurements, but it is felt that the individual panel loads permit more physical insight into the aerodynamic generation of the forces and moments. Roll moment characteristics presented are the induced roll moment, roll damping moment, and steady-state roll rate. These roll characteristics are of great importance in the dynamic flight stability of finned missiles. Control deflection forces and moments presented are pitch (or yaw) control and roll control for various control deflections and roll angles.

\section{Panel Loads}

\section{a. Panel Normal Force}

The panel normal force on the windward fin (Fin 4) as a function of roll angle for $\alpha_{b}=20^{\circ}$ for Configuration $A$ at $M_{\infty}=0.8$ is shown in Figure $31 .^{*}$ Also

\footnotetext{
* All panel loads calculated and compared with experimental data are for missile Configuration $A$.

Configuration $\mathrm{A}\left(\mathrm{M}_{\infty}=0.8\right)$
}

shown in Figure 31 is the prediction of Nielsen, Hemsch, and Smith.7 As Fin 4 sweeps from $\phi=0$ to $90^{\circ}$, it moves on the windward side of the body from the angle of attack plane to the $(x, y)$ plane. As can be seen by comparing the present result with experiment, the present method predicts very accurately the loadup of the fin. Above $\phi=60^{\circ}$, the present method predicts an increasing normal force, whereas experiment shows a slight drop in normal force. Examining the computer output for spanwise angle of attack and loading reveals that as $\phi$ increases from zero, the loading increases due to increase in local angle of attack across the span. At $\phi=40^{\circ}$, the body upwash near the fin root has increased to the extent that the root chord stalls. As the roll angle increases further, the stall location moves outboard, but the inboard sections begin to add lift as they progress further into poststall. Noting the experimental trend for $\phi>40^{\circ}$ and the rise in normal force near $\phi=80^{\circ}$ suggests the following explanation of the actual fin loading. The root chord stall is washed outboard due to the spanwise velocity component of the leading edge vortex. This, in turn, decreases the loading along the span such that the total loading decreases; however, as the roll angle increases further and the spanwise location of stall moves outboard, the portion of unstalled span that could be affected steadily decreases. At $\phi=90^{\circ}$ it is seen from the present computer results that stall occurs at the very tip of the fin; that is, the entire fin is in poststall. Once this occurs, the fin loading will begin to increase as the angle of attack increases. This hypothesis could be tested quantitatively by examining experimental spanwise pressure distributions on a fin. It could be tested qualitatively by comparing predictions of the present method with experimental data for unswept fins and noting that this phenomenon probably would not occur for unswept geometries.

The panel normal force on the windward fin vs roll angle for $\alpha_{\mathrm{b}}=20^{\circ}$ at $\mathrm{M}_{\infty}=1.22$ is shown in Figure 32. Good agreement between the present method and experiment is demonstrated in the figure. The trend in panel normal force for $\phi<50^{\circ}$ is the same for supersonic flow as the the previous subsonic case. For $\phi>50^{\circ}$, however, the experimental data show that stall is delayed to a higher roll angle (i.e., angle of attack) as compared to $\mathrm{M}_{\infty}=0.8$. Figure 32 shows the experimental data still dropping at $\phi=90^{\circ}$ which implies, from the previous discussion, that the stall angle of attack has not reached the trip chord. As the present method does not include any dependence of stall angle of attack on Mach number (Eq (35)), this characteristic is not demonstrated by theory. 
Table 1. Geometry of Missiles Used for Comparison of Theory and Experiment Configuration $b_{0} / a \quad c_{r} / a \quad x_{1} / a \quad \Lambda_{\ell}$ Ate $x_{h} / a$ Ae Reference

$\begin{array}{llllclccc}\text { A } & 2.4 & 2.8 & 17.2 & 45^{\circ} & 0^{\circ} & 18.4 & 1.33 & 27 \\ \text { B } & 2 & 1.2 & 18.1 & 0^{\circ}, & 0^{\circ} & -- & 1.67 & 28 \\ \text { C } & 2 & 1.6 & 17.7 & 38.7^{\circ} & 0^{\circ} & -- & 1.67 & 28 \\ \text { D } & 2 & 2.4 & 16.9 & 67.4^{\circ} & 0^{\circ} & -- & 1.67 & 28 \\ \text { E } & 3 & 2 & 18.0 & 0^{\circ} & 0^{\circ} & -- & 2.00 & 10,29 \\ \text { F } & 2 & 2.66 & 17.34 & 53.1^{\circ} & 0^{\circ} & -- & 1.00 & 30\end{array}$

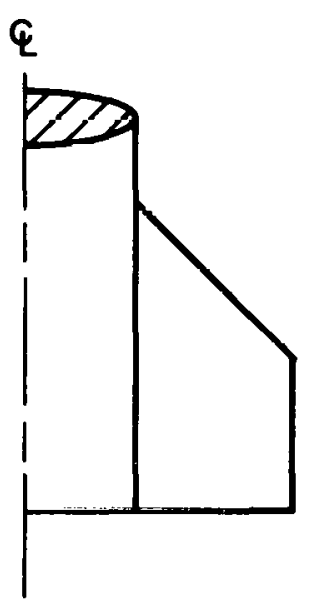

CONFIG. A

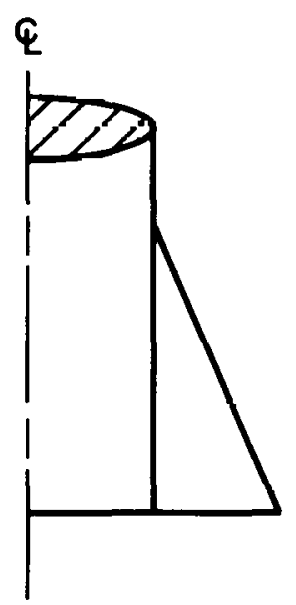

CONFIG. D

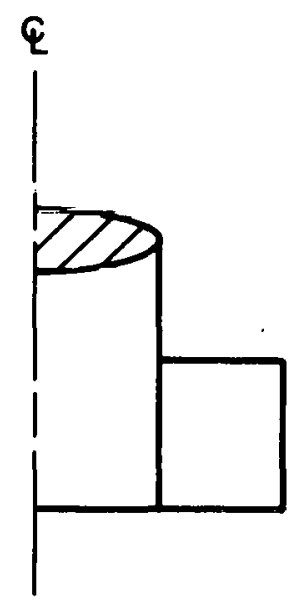

CONFIG. B

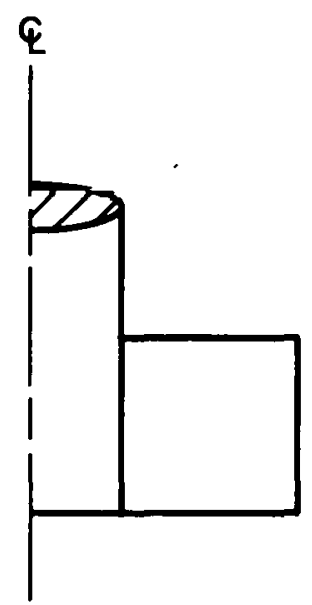

CONFIG. E

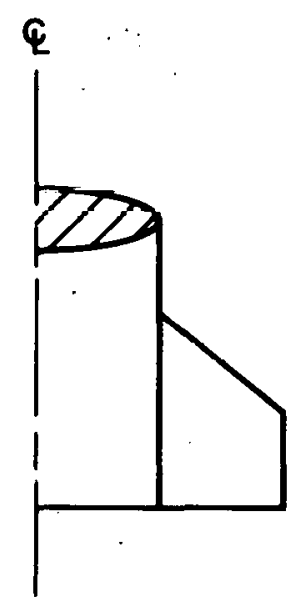

CONFIG. C

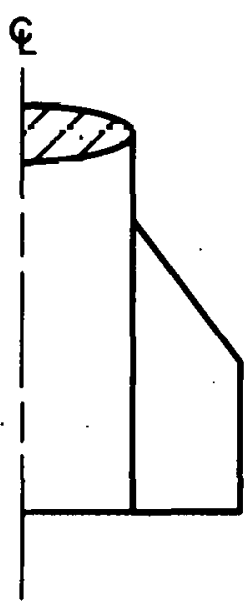

CONFIG. F

Figure 30. Fin Plantorms Used for Comparison of 'Theory and Experiment 


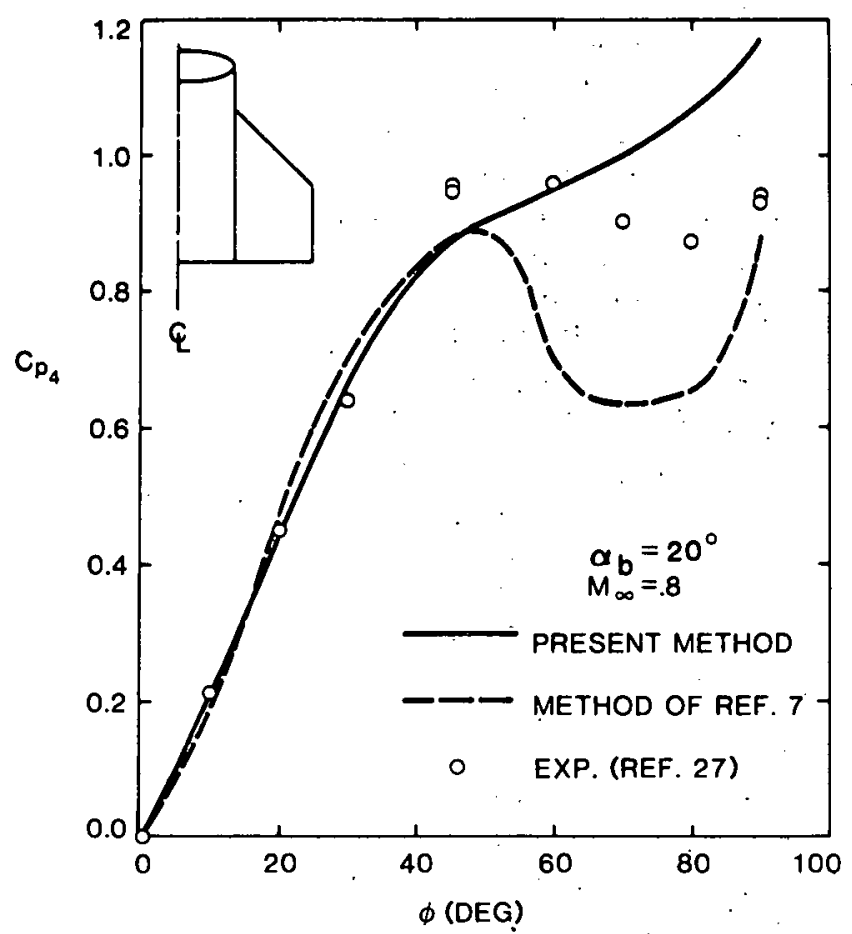

Figure 31. Windward Panel Normal Force vs Roll Angle for Configuration $\mathrm{A}\left(\mathrm{M}_{\infty}=0.8\right)$

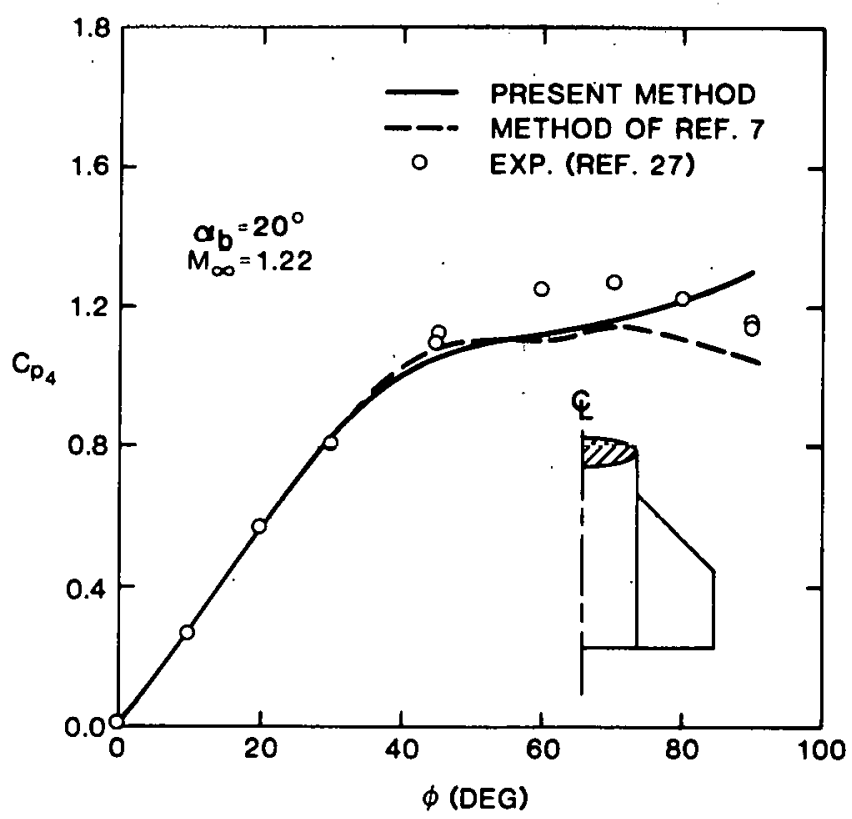

Figure 32. Windward Panel Normal Force vs Roll Angle for Configuration $\mathrm{A}\left(\mathrm{M}_{\infty}=1.22\right)$
The panel normal force on the leeward fin vs roll angle for $\alpha_{b}$ at $M_{\infty}=0.8$ is given in Figure 33. Very good agreement between theory and experiment is demonstrated except near $\phi=0^{\circ}$. As Fin 1 rolls from $\phi=0^{\circ}$, it begins to unload not only because of closer proximity to the angle of attack plane, but also immersion in the body vortex. The inboard portion of the fin unloads much more rapidly than the outboard portion because it is strongly influenced by the vortex feeding sheet. As the fin nears the center of the body.. vortex it produces essentially no net normal force. The portion inboard of the vortex produces negative force (i.e., in the negative roll moment direction) and the outboard portion produces an almost balancing positive force. This balance is highly dependent on the relation between the radial vortex location and the fin semispan. For example, if the fin had a smaller semispan, the zero load roll angle would be less than that shown in Figure 33 .

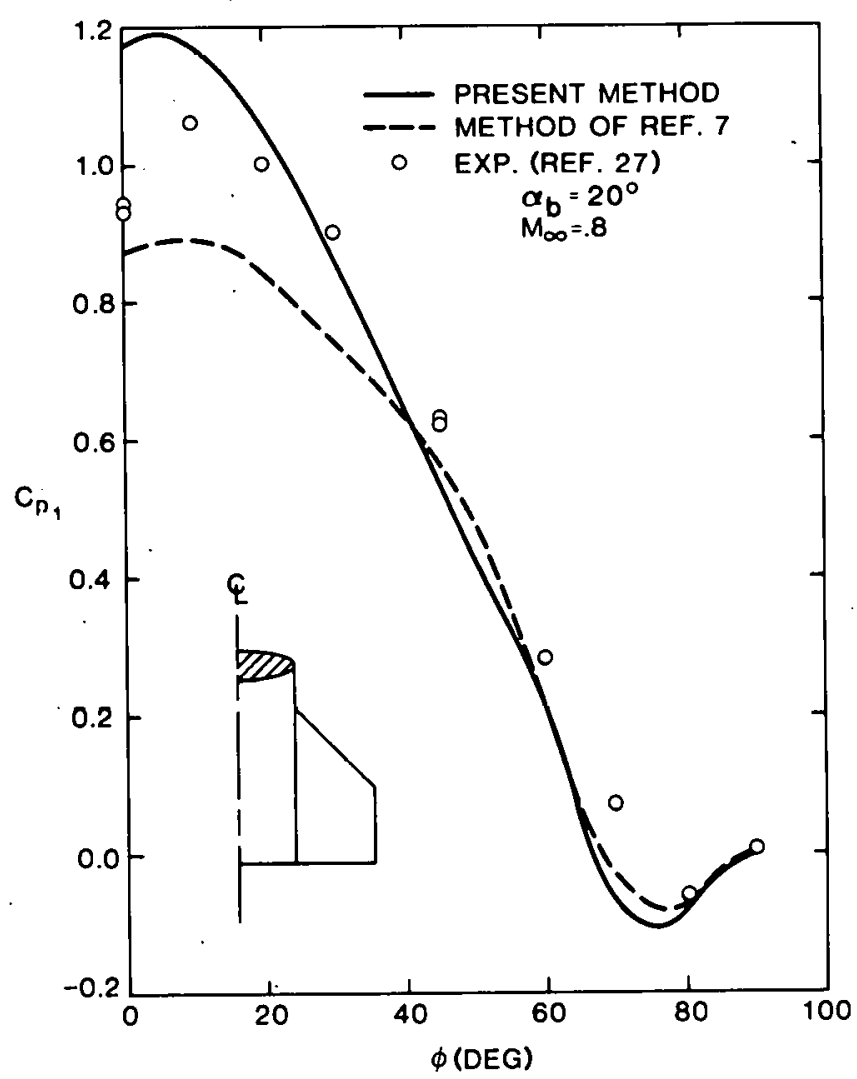

Figure 33. Leeward Panel Normal Force vs Roll Angle for Configuration $\mathrm{A}\left(\mathrm{M}_{\infty}=0.8\right)$ 
Figure 34 shows the same type of comparison as Figure 33, except for $M_{\infty}=1.22$. The agreement between theory and experiment is not as good as in the previous comparisons. Note that the experimental roll angle for zero cross-over loading decreased from $\phi=75^{\circ}$ for $\mathrm{M}_{\infty}=0.8$ to $\phi=60^{\circ}$ for $\mathrm{M}_{\infty}=1.22$. This could be caused by the body vortex becoming stronger or the radial location of the vortex increasing, or both. Recalling that the radial location of the vortex increases with Mach number (Eq (11)), one could infer that vortex strength increases significantly with Mach number. This has been suggested by Nielsen in Reference 26.

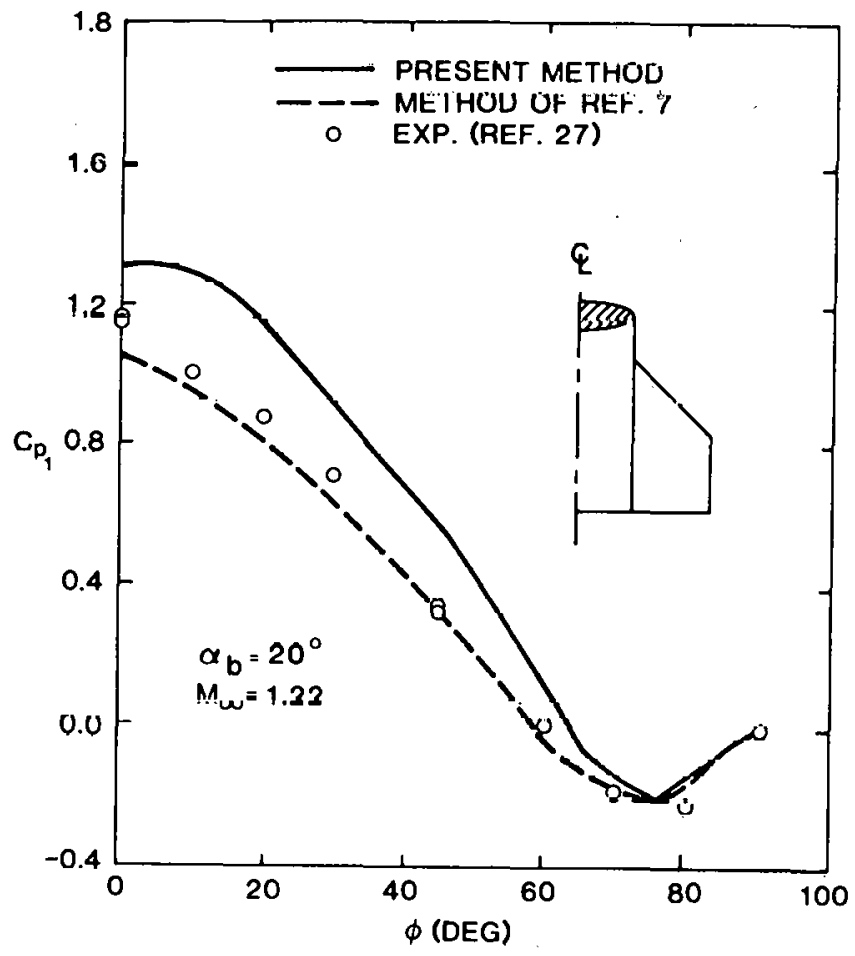

Figure 34. Leeward Panel Normal Force vs Roll Angle for Configuration $\mathrm{A}\left(\mathrm{M}_{\infty}=1.22\right)$

\section{b. Panel Bending Moment}

Figures 35 and 36 show the panel bending moment for the windward fin vs roll angle for $\alpha_{b}=20^{\circ}$ at $\mathrm{M}_{\infty}=0.8$ and 1.22 , respectively. Generally good agreement is demonstrated between theory and experiment. Good agreement between theory and experiment on the bending moment requires that both the magnitude of the total normal force, $C_{p_{4}}$, and spanwise pressure distribution are correct. Recall that the theory for panel normal force is in essentially perfect agreement with experiment (Figures 31 and 32) for $\phi<40^{\circ}$. Figures 35 and 36, however, show that the theory slightly underpredicts the bending moment for the same roll angle range. This implies that there is slightly more loading outboard and slightly less loading inboard than predicted by the present method. If bending moment data for other planforms indicated the same trend, the spanwise normal force distribution, $S(\eta)$, given by $\mathrm{Eq}(19 \mathrm{~b})$ could be modified slightly.

The root bending moment for the leeward fin vs roll angle for $\alpha_{b}=20^{\circ}$ at $M_{\infty}=0.8$ and 1.22 is shown in Figures 37 and 38, respectively. Excellent agreement between theory and experiment is demonstrated for the subsonic case, and fair agreement is shown for the supersonic case. It is interesting that for $\mathrm{M}_{w}=$ 0.8 near $\phi=80^{\circ}$, the panel normal force is negative (Figure 33) while the bending moment remains near zero (Figure 37). The reason for this is that the reverse flow under the vortex is strong enough to generate a net negative panel force, but the bending moment produced by the outboard positive normal force overcomes the negative bending moment produced by the inboard sections.

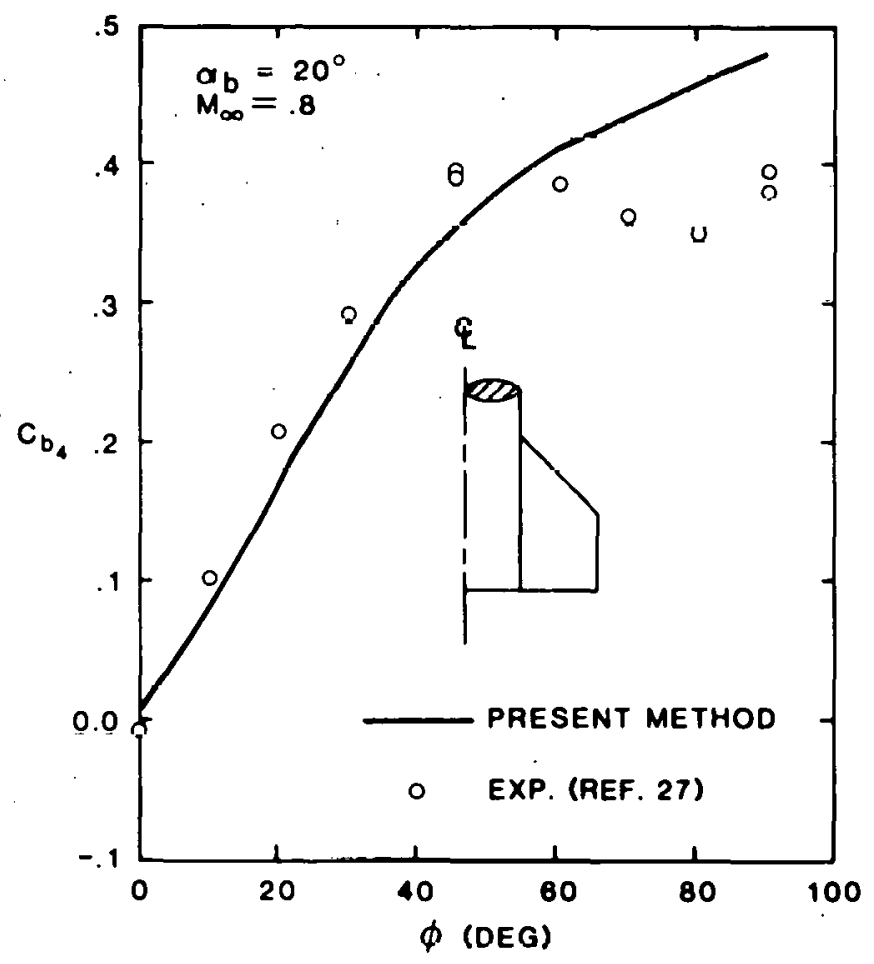

Figure 35. Windward Fin Root Bending Moment vs Roll Angle for Configuration $A\left(M_{\infty}=0.8\right)$ 


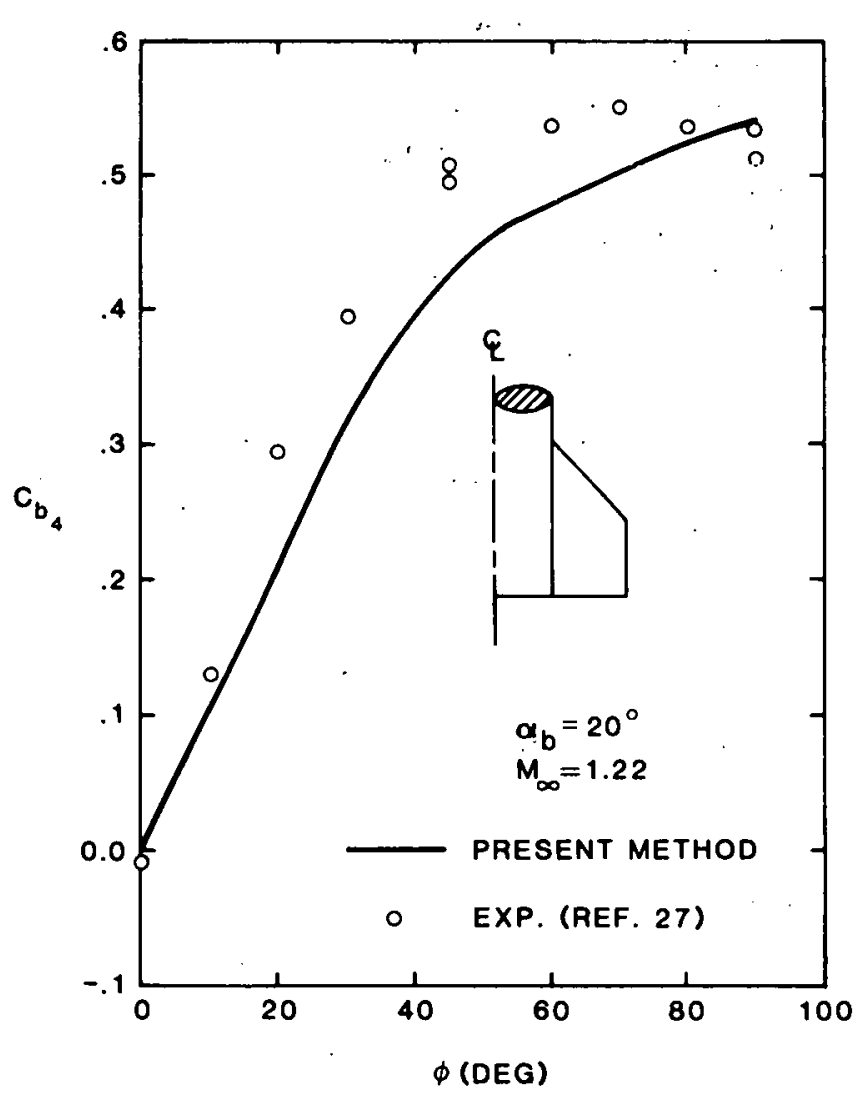

Figure 36. Wiridward Fin Root Bending Moment vs Roll Angle for Configuration $A\left(M_{\infty}=1.22\right)$

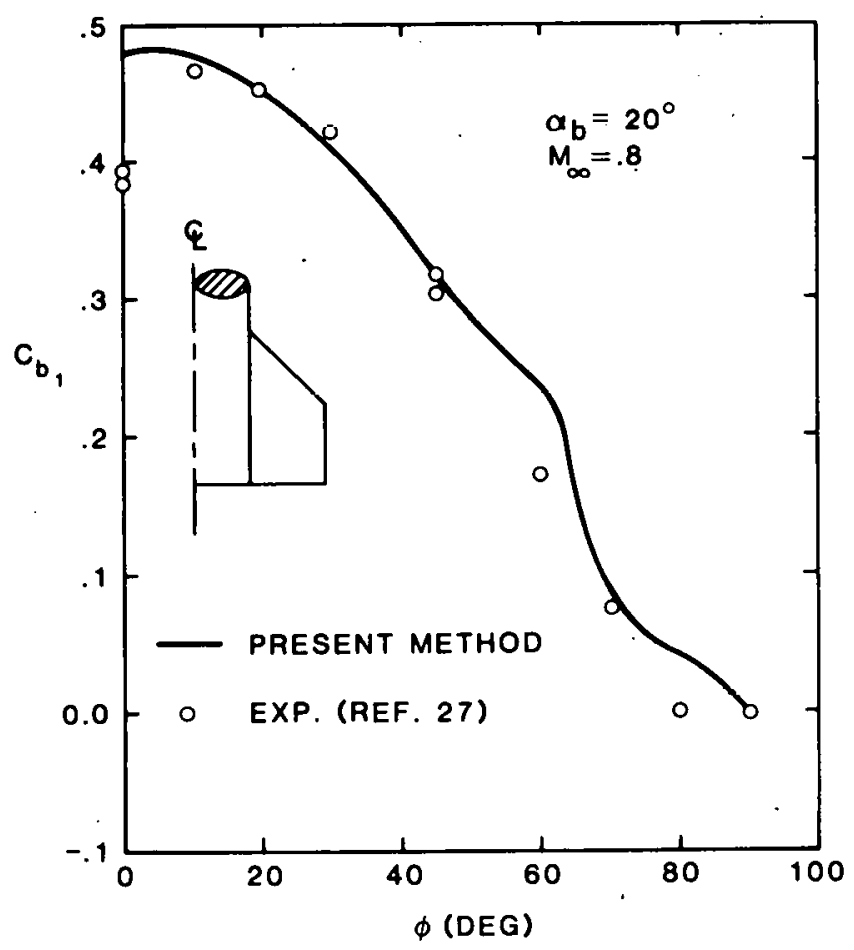

Figure 37. Leeward Fin Root Bending Moment vs Roll Angle for Configuration $\mathrm{A}\left(\mathrm{M}_{\infty}=0.8\right)$

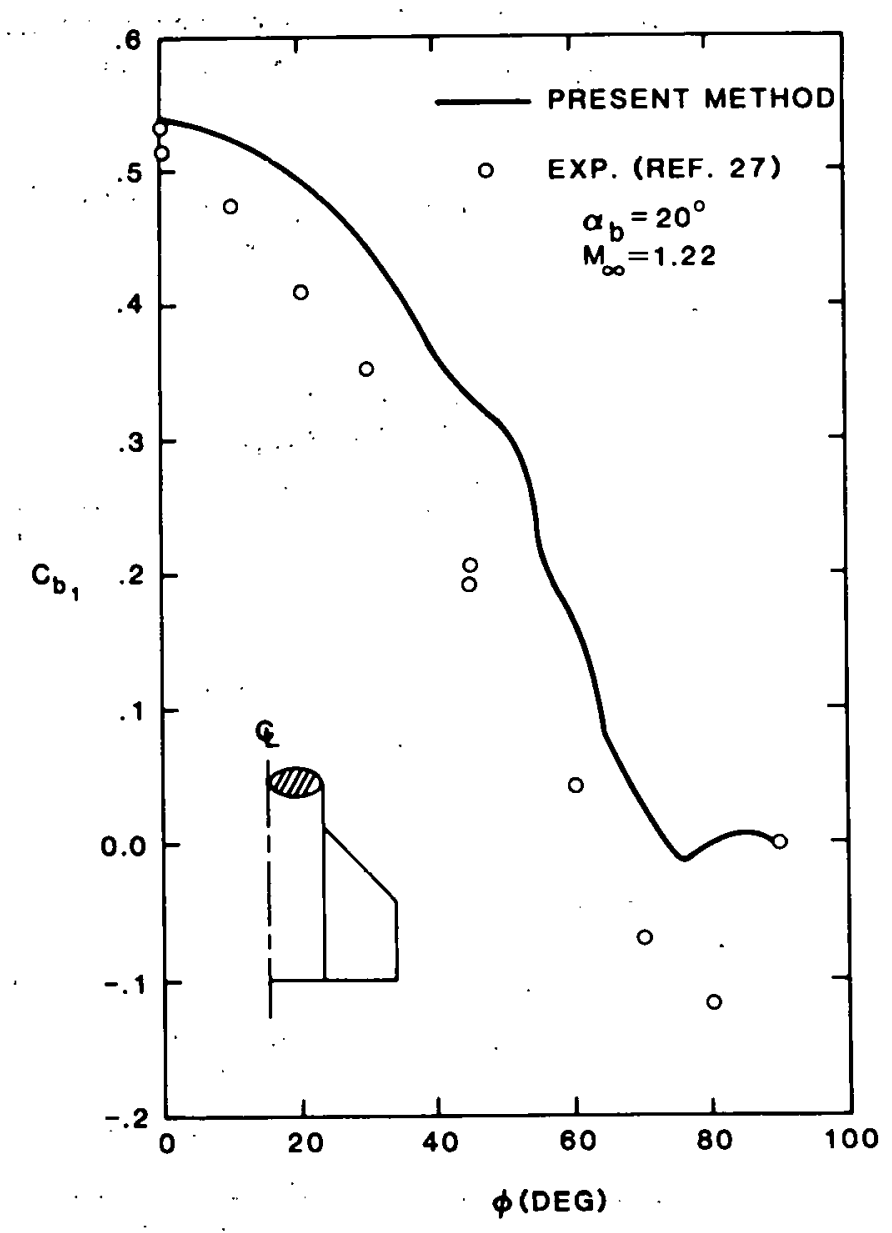

Figure 38. Leeward Fin Root Bending Moment vs Roll Angle for Configuration $\mathrm{A}\left(\mathrm{M}_{\infty} \doteq 1.22\right)$

\section{c. Panel Hinge Moment}

The panel hinge moment for the windward fin vs roll angle for $\alpha_{\mathrm{b}}=20^{\circ}$ at $\mathrm{M}_{\infty}=0.8$ and 1.22 is shown in Figures 39 and 40 , respectively. The agreement between theory and experiment appears to be poor, particularly for $\mathrm{M}_{\infty}=1.22$. One must be aware, however, of the sensitivity of the hinge moment to the location of the hinge line. A better indication of the accuracy of the present method is obtained by a sample comparison of the theoretical and experimental axial location of the fin center of pressure. Using the hinge line, $x_{h}$, as the moment reference, one can write

$$
x_{c p} C_{p} q_{\infty} S_{f}=H
$$

Solving for the location of the center of pressure, nondimensionalized by the root chord, one has

$$
x_{c p} / c_{r}=C_{h} / C_{p}
$$


Applying this equation to both the experimental and theoretical data at $\phi=90^{\circ}$ for Fin 4, one has

For $\mathrm{M}_{\infty}=0.8$

$\left(\frac{x_{c p}}{c_{r}}\right)_{\exp }=0.113$ and $\left(\frac{x_{c p}}{c_{s}}\right)_{\text {theory }}=0.023$

For $\mathrm{M}_{\infty}=1.22$

$\left(\frac{x_{c p}}{c_{r}}\right)_{\exp }=0.129$ and $\left(\frac{x_{c p}}{c_{r}}\right)_{\text {theory }}=0.024$

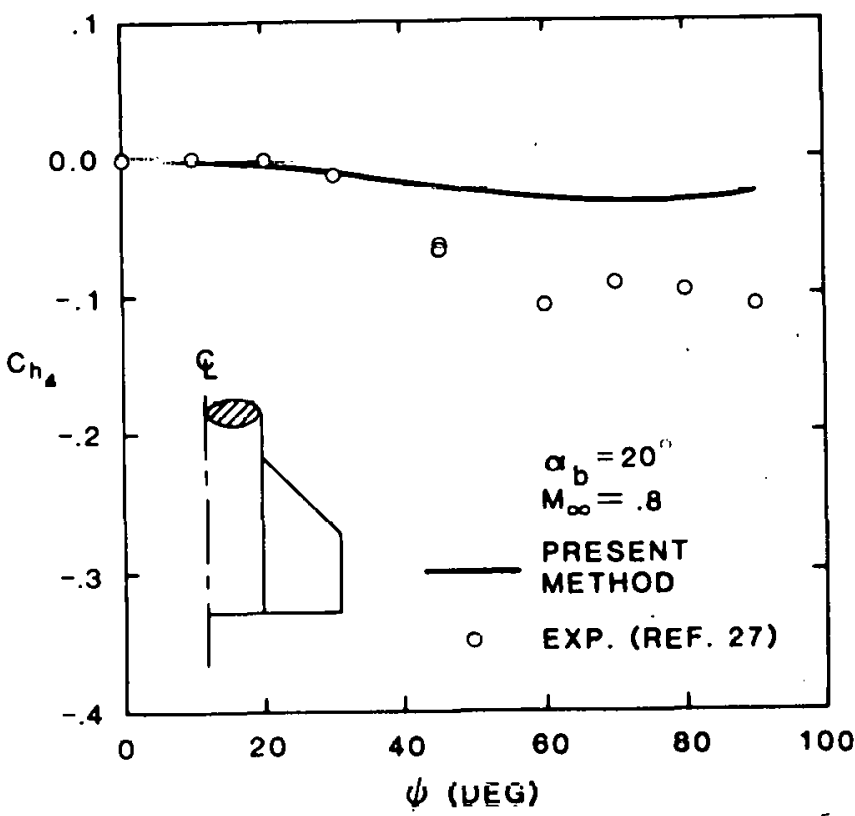

Figure 39. Windward Fin Hinge Moment vs Roll Angle for Eunfiguratlun $A\left(\mathrm{Ml}_{\infty}=0.8\right)$

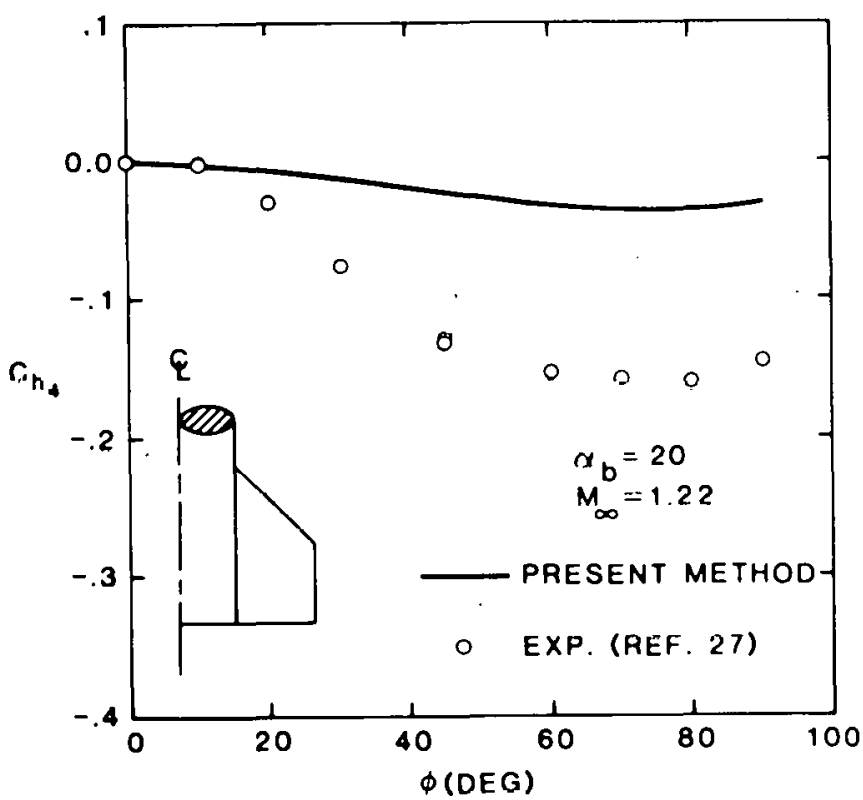

Figure 40. Windward Fin Hinge Moment vs Roll Angle for Configuration $\mathrm{A}\left(\mathrm{M}_{\infty}=1.22\right)$
As can be seen by this calculation, the experimental center of pressure is slightly farther aft of the hinge line than predicted by theory. Taking the difference between each pair of ratios, the predicted axial center of pressure is in error only $9.0 \%$ and $10.5 \%$ of the root chord for $\mathrm{M}_{\infty}=0.8$ and 1.22 , respectively, for the worst agreement between theory and experiment in Figures 39 and 40.

A second point should be made from the axial center of pressure calculation just presented. Note that the experimental data show that the center of pressure moves slightly rearward as the Mach number changes from subsonic to supersonic. This follows the usual trend of lifting surfaces in transonic flow. The predicted center of pressure, however, stays essentially constant with the Mach number; the reason is that the assumed normal force distribution for uniform apprinact flow (Fq (19)) dnes not depend upon the Mach number. A slight improvement to the present theory would be to insert Mach number dependence in the chordwise distribution function, $C(\xi)$.

Figures 41 and 42 give the hinge moment for the leeward fin vs roll angle for $\alpha_{\mathrm{b}}=20^{\circ}$ at $\mathrm{M}_{\infty}=0.8$ and 1.22 , respectively. Better agreement between theory and experiment is shown for the leeward fin than for the windward fin. This might be somewhat surprising because of the complexity of predicting fin loading for such a nonuniform approach flow. The reason is that for $\phi>50^{\circ}$ the panel normal force is small for the lecward fin; therefore, the hinge moment is, also small.

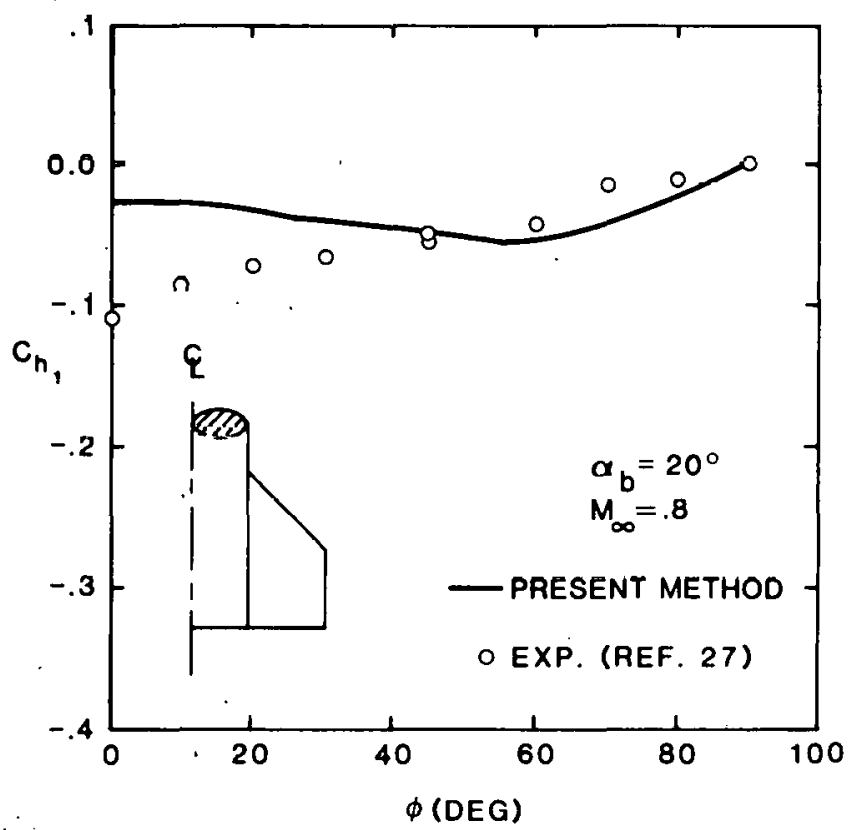

Figure 41. Leeward Fin Hinge Moment vs Roll Angle for Configuration $\mathrm{A}\left(\mathrm{M}_{\infty}=0.8\right)$ 


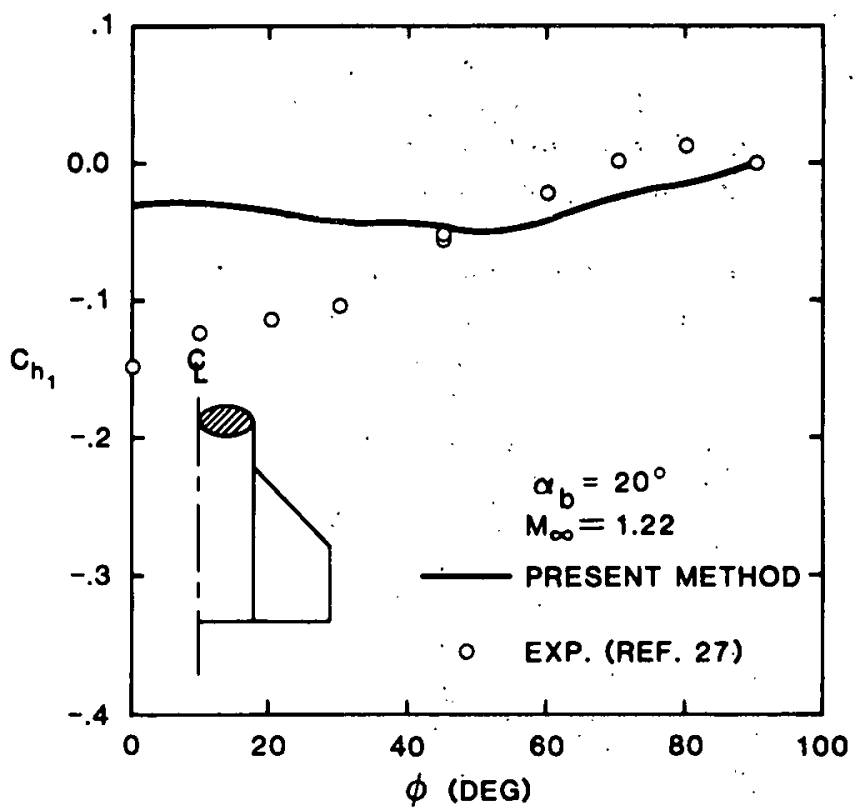

Figure 42. Leeward Fin Hinge Moment vs Roll Angle for Configuration $\mathrm{A}\left(\mathrm{M}_{\infty}=1.22\right)$

\section{Roll Moments}

\section{a. Induced Roll Moment}

Figures 43 and 44 show the induced roll moment vs angle of attack of the body for missile Configuration $\mathrm{A}$ for $\phi=20^{\circ}$ at $\mathrm{M}_{\infty}=0.8$ and $\mathrm{M}_{\infty}=1.22$, respectively. Also shown in the figures is the prediction of Nielsen, Hemsch, and Smith.7 Fairly good agreement is observed between the present method and experiment for $\mathrm{M}_{\infty}=0.8$, but for $\mathrm{M}_{\infty}=1.22$, the agreement is poor. Note that the induced roll moment is one of the most difficult nnnlinear moments to predict because it is composed of the sum and difference of the first moment of four spanwise pressure distributions. The physical explanation of the slightly negative then rapidly increasing positive trend of the induced roll moment with angle of attack is very difficult to delineate because of the many complex aerodynamic phenomena embedded in the present theory. From numerical experiments with the thenry, however, certain important elements can be identified: radial location of the body vortex, size of the vortex core, and local stall and poststall along the span of the fin.

To understand the trends plotted in Figures 43 and 44 , one must examine the spanwise loading of all the fins. Figure 45 shows the spanwise loading for each fin for $M_{\infty}=0.8$. The loading at a given spanwise location shown in Figure 45 is the integrated value over the local chord. The loading caused by the primary body vortex is clearly seen on Fin 2 . The spanwise location of the stall angle of attack can be recognized as a slight drop in the normal force along Fin 3. The loading on Fins 1, 3, and 4 increases steadily as the root chord is approached because of body upwash and the increasing length of the chord. On Fin 1 the loading drops sharply near the root chord because it passes inside the vortex feeding sheet. The roll moment produced by each fin loading shown is: Fin 1, 0.794; Fin 2, -0.031; Fin 3, -0.751; and Fin 4, 0.313. It can be seen that the roll moments produced by Fin 1 and Fin 3 roughly balance. Fin 2 and Fin 4, however, do not balance because the reverse flow loading between the primary body vortex and the root chord on Fin 2 drop (in magnitude) the roll moment on Fin 2 to roughly zero. This results in a large net positive roll moment from Fin 4. By similar reasoning, the small negative induced roll moment for small angles of attack is due to the increased outboard loading on Fin 2 when the vortex is near the body surface.

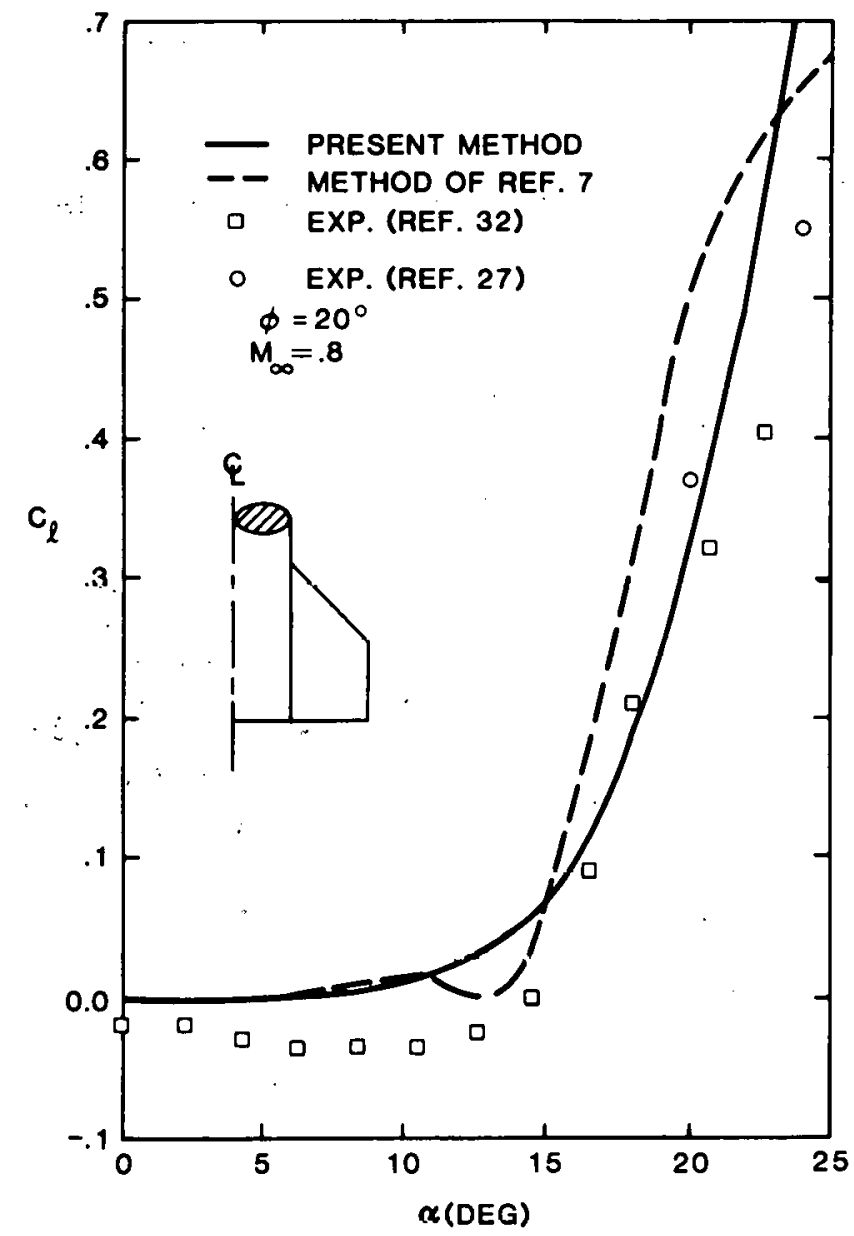

Figure 43. Induced Roll Moment vs $\alpha_{b}$ for Configuration $A\left(M_{\infty}\right.$ $=0.8$ ) 


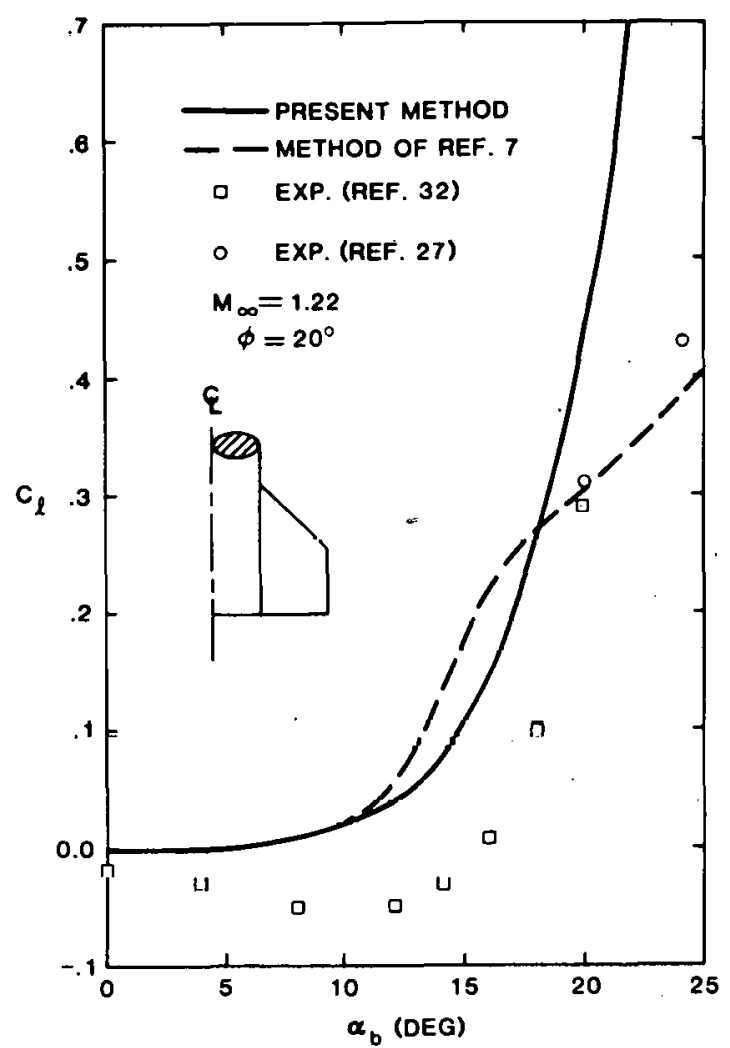

Figure 44. Induced Roll Moment vs $\alpha_{b}$ for Configuration $A\left(M_{\infty}\right.$ $=1.22$ )

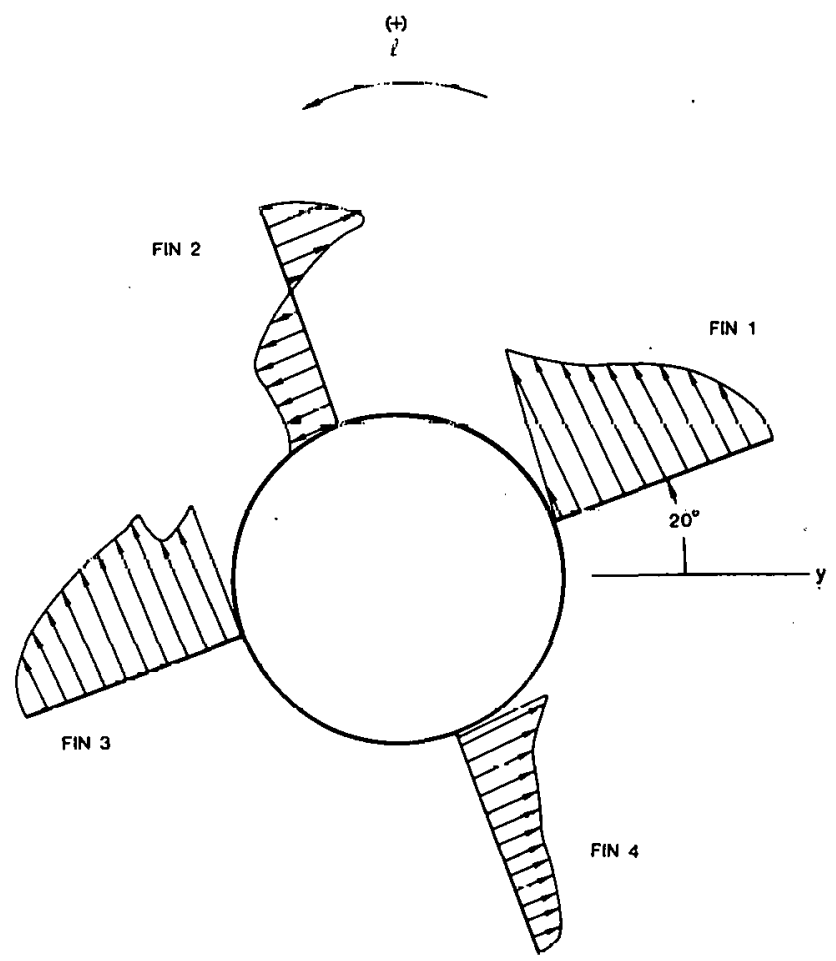

Figure 45. Spanwise Fin Loading for $\alpha_{b}=20^{\circ}, \phi=20^{\circ}$ Configuration $\mathrm{A}\left(\mathrm{M}_{\infty}=0.8\right)$
Figure 46 gives the induced roll moment vs angle of attack for Configuration $B$ for $\phi=22.5^{\circ}$ and $M_{\infty}=$ 0.7 . Theory and experiment are in very good agreement for the rectangular fin planform. Comparing the induced roll moment coefficient for the clipped delta and rectangular planform (Figures 43 and 46) for $\alpha_{b}=20^{\circ}$, it is seen that ${ }^{*} C_{\ell}=0.325$ and 0.186 , respectively. Recalling the previous explanation given concerning the origin of the large positive induced roll moment, one could ask why $C_{\ell}$ for the shorter span rectangular planform is not larger than that for the clipped delta. The reason for the question would be that the reverse flow loading on Fin 2 for the short span fin should produce a relatively larger positive roll moment than would the clipped delta. The reasoning is correct; the paradox, however, is created by the nondimensionalization used in the preaent annlyais and in nimnat all other analyaes The roll moment coefficient is based on the body crosssectional area, not on the fin planform area. If one converts the two previous coefficients to coefficients based on fin planform area, one has $\left[\mathrm{C}_{\ell}\right]_{\mathrm{S}_{\mathrm{f}}}=0.347$ and 0.487 , respectively. Now it is clear that the short span rectangular planform is much more efficient at producing an induced roll moment than the longer span clipped delta.

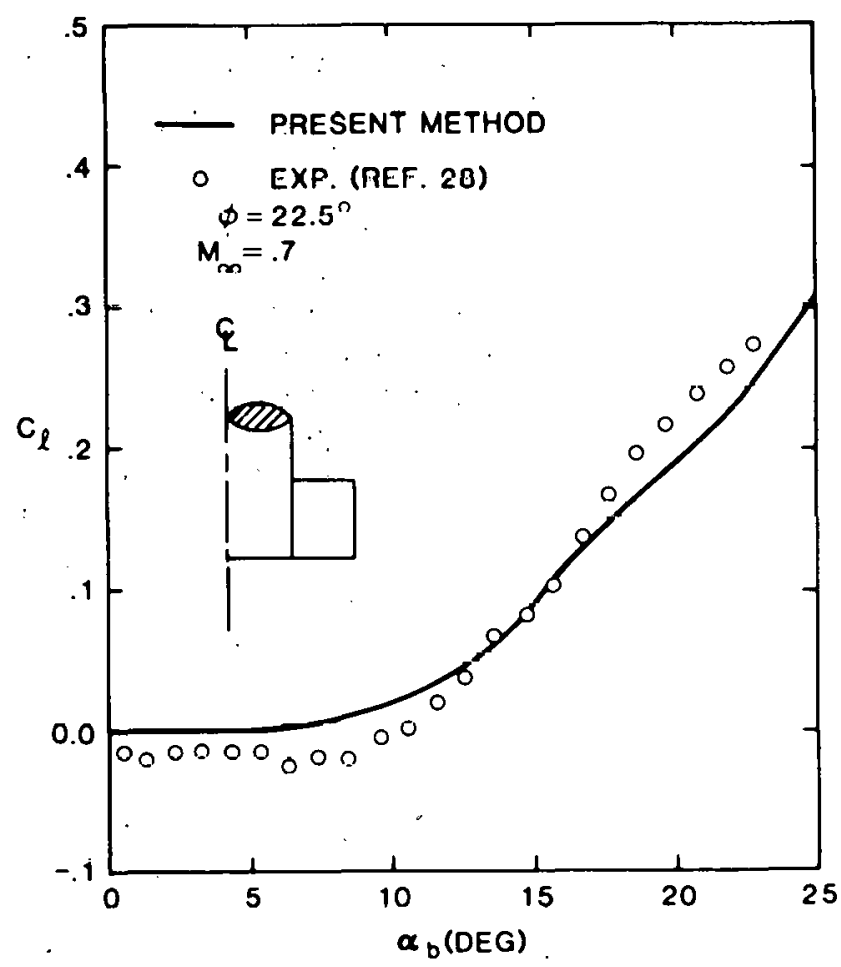

Figure 46. Induced Roll Moment vs $\alpha_{b}$ for Configuration B

\footnotetext{
*This comparison ignores the fact that the roll angle and Mach number are not exactly the same.
} 
The induced roll moment vs angle of attack for Configuration $D$ for $\phi=22.5^{\circ}$ and $M_{\infty}=0.7$ and 1.2 is shown in Figure 47 . The theory reproduces the expcrimental data fairly well, but the change in induced roll moment with Mach number is not predicted accurately for this planform geometry. The reason is probably the effect of Mach number on the spanwise and chordwise pressure distributions, $S(\eta)$ and $C(\xi)$, as mentioned earlier. Concerning the magnitude of the induced roll moment coefficient, a direct comparison can be made between the rectangular planform (Figure 46) and the delta planform (Figure 47) because they both have the same missile body and planform area. The delta planform produces a slightly smaller induced roll moment because of the greatly different spanwise loading and stall angle of attack (compare, for example, Figures 17 and 19).

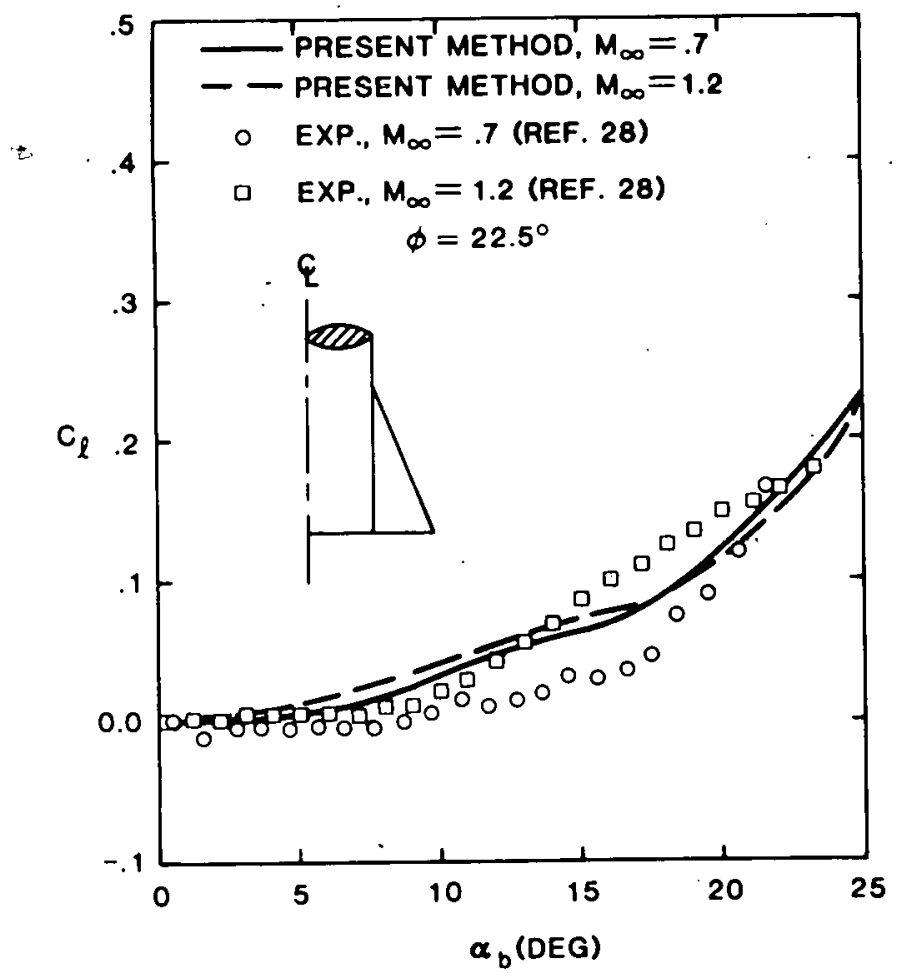

Figure 47. Induced Roll Moment vs $\alpha_{b}$ for Configuration D

\section{b. Roll Damping Moment}

Figure 48 shows the roll damping moment vs angle of attack for Configuration $\mathrm{E}$ for $\mathrm{M}_{\infty}=0.22$. There is excellent agreement between theory and experiment for angles of attack up to $12^{\circ}$, but above that the theury agrees well with one set of experimental data and not the other. The only comment that can be made is that the experimental techniques of the two investigators differ greatly. Clare ${ }^{31}$ used a roll oscillation technique; Regan ${ }^{29}$ used the standard rolling-speed decay method.

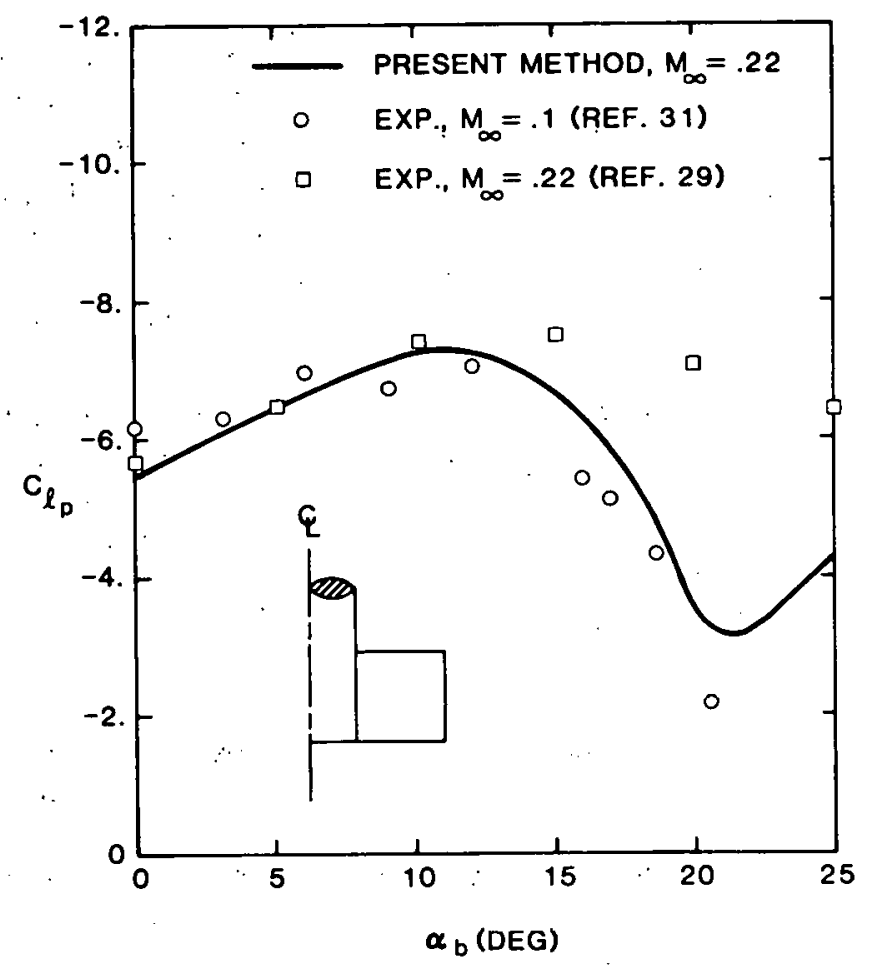

Figure 48. Roll Damping Moment vs $\alpha_{\mathrm{b}}$ for Configuration E.

A comment should be made concerning the present numerical method of calculating dynamic derivatives, such as $C_{\ell_{p}}$. As the missile fins rotate through a $90^{\circ}$ roll cycle, the forces and moments continually change for nonzero angle of attack of the body. Therefore, one method of computing rolling motion derivatives is to numerically difference the average value of the force or moment coefficient over a roll cycle; that is,

$C_{i_{p}}=\frac{2}{\pi}\left[\left.\int_{0}^{\pi / 2} C_{i}\right|_{p=\Delta p} d \phi-\left.\int_{0}^{\pi / 2} C_{i}\right|_{p=0} d \phi\right] / \Delta p$

where $C_{i}$ represents any force or moment coefficient. This computation of $C_{i_{p}}$ is appropriate for large values of rolling speed. The method described by Eq (56) is one used in the present analysis. It should be mentioned, however, that another method could be used. This method computes the difference between two values of the coefficient at different rolling speeds, but at the same roll angle; that is

$\left.C_{i_{p}}\right|_{\phi=\phi_{1}}=\left[\left.C_{i}\right|_{\substack{\phi=\phi_{1} \\ p=\Delta p}}-\left.C_{i}\right|_{\substack{\phi=\phi_{1} \\ p=0}}\right] / \Delta p$. 
This method could be used for very low rolling speeds, such as roll oscillations about a roll trim angle; it would yield, for example, the roll angle variation of the roll damping moment while a missile is oscillating in roll lock-in.

Figure 49 gives the roll damping moment vs angle of attack for Configuration $F$ for $M_{\infty}=0.6$ and 1.3. Although the slight drop in $C_{\ell_{p}}$ near $\alpha_{b}=17^{\circ}$ is not predicted, the agreement between theory and experiment is generally good over the angle of attack range. Comparing Figures 48 and 49 , it is seen that the trend of $C_{\ell_{p}}$ with $\alpha_{b}$ is very different between the two planforms. One might suspect, based on the earlier discussion of induced roll moment, that this different character would be due primarily to the difference in semispan between the two configurations. The roll damping moment for several fin planforms and semispans was computed in order to identify the dominant parameter causing the rapid decrease in $C_{\ell_{p}}$ for $\alpha_{b}$ near $20^{\circ}$; it was the stall angle of attack of the planform and not fin span. For example, consider the case of holding the planform and span constant (say a rectangular planform of a given span), and varying the aspect ratio so that the stall angle of attack varies. The aspect ratio decreases (and the stall angle of attack increases) as the drop in $\mathrm{C}_{\ell_{\mathrm{p}}}$ at large angle of attack disappears.

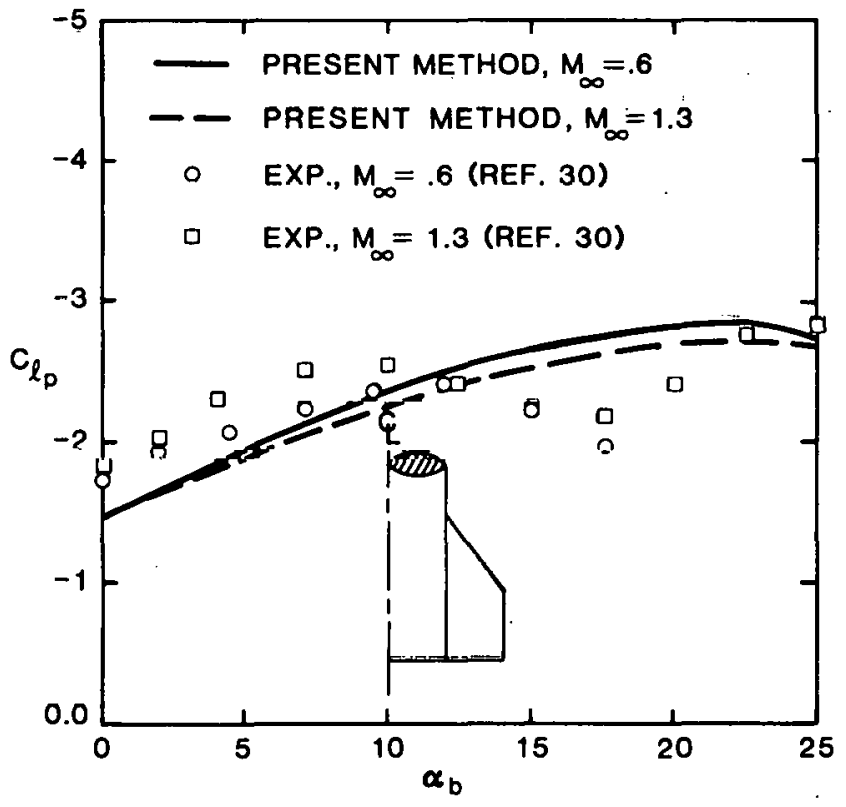

Figure 49. Koll Damping Moment vs $\alpha_{b}$ for Configuration F.

\section{c. Steady-State Rolling Speed}

The steady-state roll rate of a missile whose fins are canted is calculated in a manner similar to $C_{\ell_{\mathrm{p}}}$. The steady-state roll rate is defined as the rolling speed at which the roll driving moment balances with the roll damping moment. Therefore, the nondimensional steady-state roll rate, $\mathrm{p}_{\mathrm{ss}}$ is the roll rate such that

$$
\int_{0}^{\frac{\pi}{2}}\left[C_{\ell}\right]_{p \circ p_{s s}} d \phi=0 .
$$

Only one comparison of theory and experiment is made for steady-state rolling speed.because of the lack of data for other planforms. Figure 50 shows the steady-state rolling speed vs angle of attack for Configuration $E$ for a fin cant of $4^{\circ}$ (differential fin deflection) and $\mathrm{M}_{\mathrm{m}}=0.23$. The theory slightly overpredicts $p_{s s}$ for angles of attack less than $12^{\circ}$, but for $\alpha_{b}>12^{\circ}$ the theory agrees perfectly with one set of data and underpredicts $p_{s s}$ for the other set. The reason the theory predicts roll slowdown is rather difficult to determine. From numerical experiments with the present method it was found that roll slowdown was predicted for each of the planforms shown in Figure 30. One qualitative comment that can be made, however, is that even though the roll damping moment decreases at large angle of attack (Figure 48), the roll driving moment (i.e., tin cant) decreases at a faster rate on every planform investigated.

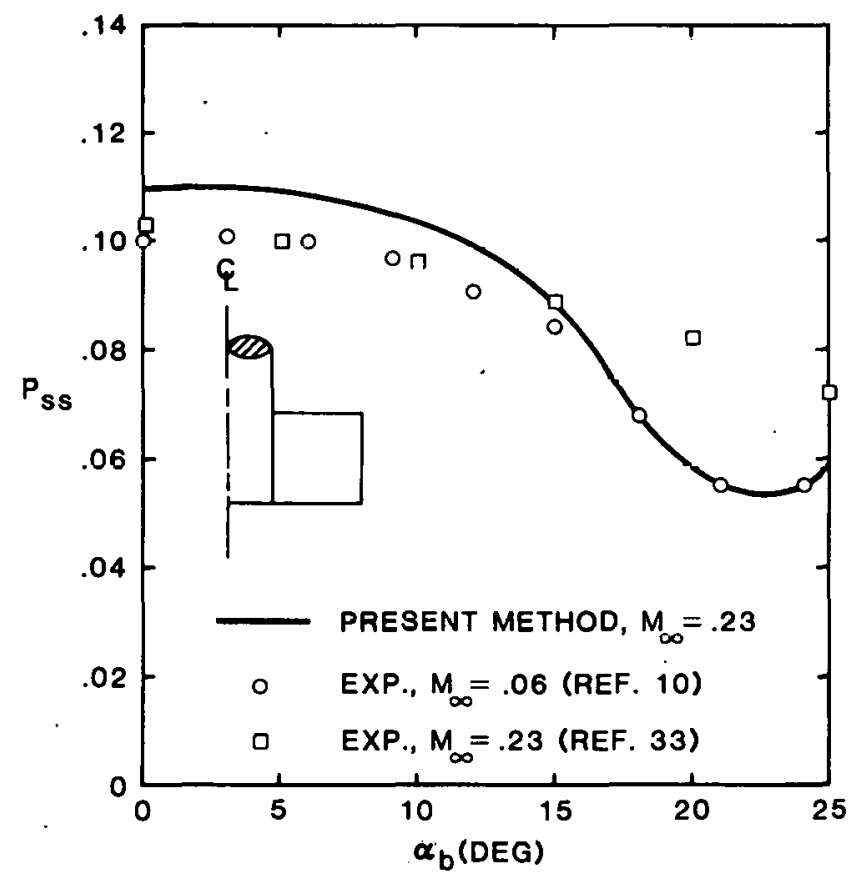

Figure 50. Steady-State Roll Rate vs $\alpha_{\mathbf{b}}$ for Configuration $\mathrm{E}\left(4^{\circ}\right.$ fin cant) 


\section{Control Deflections}

\section{a. Pitch (or Yaw) Control}

Pitch control force coefficient is defined as the normal force coefficient of the entire missile configuration (body plus fins) with the fins deflected minus the normal force coefficient of the entire missile without the fins deflected; that is,

$$
\Delta \mathrm{C}_{\mathrm{z}}=\left[\mathrm{C}_{\mathrm{z}_{\mathrm{B}+\mathrm{i}}}\right]_{\delta_{j} \neq 0}-\left[\mathrm{C}_{\mathrm{z}_{\mathrm{B}+\mathrm{f}}}\right]_{\delta_{\mathrm{j}}-0} .
$$

Using slender body theory notation, one can write

$$
\begin{aligned}
\Delta C_{z} & =\left[C_{z_{B(A)}}\right]_{\delta_{j} \neq 0}+\left[C_{z_{(B)}}\right]_{\delta_{j} \neq 0} \\
& -\left[C_{z_{B(N)}}\right]_{\delta_{j}=0}-\left[C_{z_{(B)}}\right]_{\delta_{j}=0} .
\end{aligned}
$$

The second and fourth terms are computed in the present analysis, but the first and third are not. The first term can be segregated into two terms:

$$
\left[C_{z_{B(1)}}\right]_{\delta_{j} \neq 0}=\left[C_{z_{B(i)}}\right]_{\delta_{j}=0}+C_{z_{B<\delta_{j} p^{\prime}}}
$$

where the second term on the right side of the equation is the normal force coefficient of the body due to control deflection of the fins. Substituting Eq (56) into Eq (55), one has' '

$$
\Delta C_{z}=C_{z_{B<\delta}>}+\left[C_{\left.z_{(B B}\right)}\right]_{\delta_{j} \neq 0}-\left[C_{z_{(\mathcal{B})}}\right]_{\delta_{j}=0}
$$

A simple result of slender body theory is used to estimate $C_{z_{B<\delta_{1}>}}$. Using the definition of the interference lift ratio, $k_{B}$ (see Ref. 24, pp 213-218), one has

$\mathrm{C}_{\mathrm{z}_{\mathrm{B}<\delta_{j}>}}=\mathrm{k}_{\mathrm{B}}\left[\mathrm{C}_{\left.\mathrm{z}_{\mathbf{z}(\mathrm{B})}\right]_{\delta_{i} \neq 0}}\right.$.

Substituting this into Eq (57), one has

$$
\Delta C_{z}=\left[1+k_{B}\right]\left[C_{z_{f(B)}}\right]_{\delta_{j} \neq 0}-\left[C_{z_{f(B)}}\right]_{\delta_{j}=0}
$$

The term $k_{B}$ is calculated from slender body theory assuming that the angle of attack of the body is zero. One could use Eq (58) to calculate $\Delta C_{z}$ for any angle of attack and neglect the inconsistency between this and the derivation of $k_{B}$. The present analysis, however, chooses the approach of segregating the $\alpha_{b}=0$ fin deflection interference and then using this constant value for nonzero $\alpha_{\mathrm{b}}$. Rewriting Eq (58) according to this approach, we have

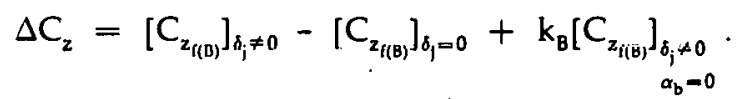

Equation (59) was used in the present analysis for computing pitch control forces with fin-body interference. $k_{B}$ is a simple function of $a / b_{0}$ and is plotted in Figure 51.

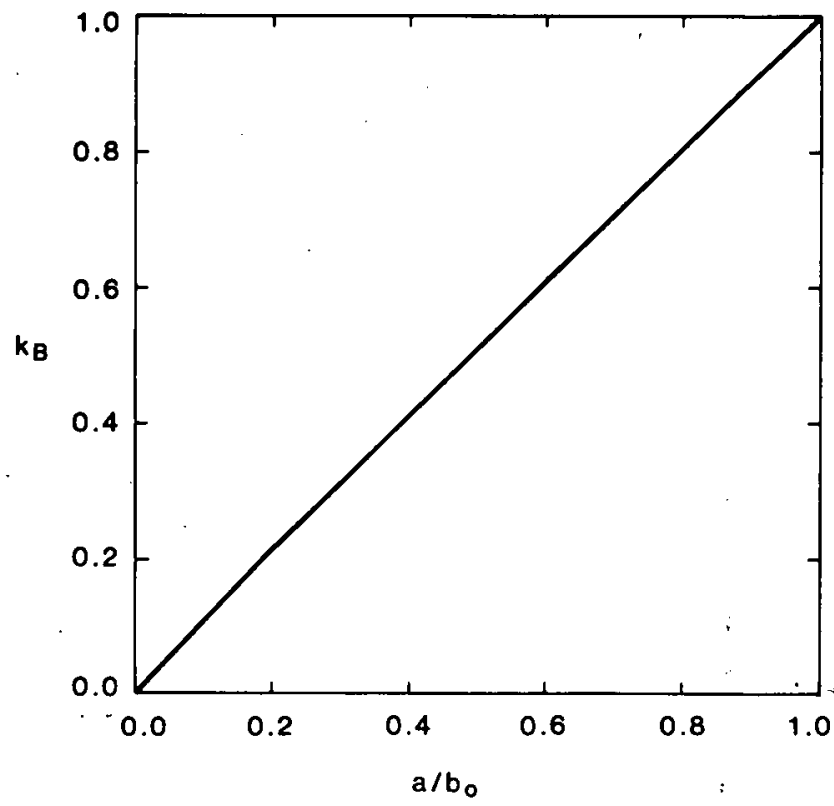

Figure 51. Interference Lift Ratio vs $a / b_{0}$ (from Ref. 24)

Figures 52, 53, and 54 show the pitch control force versus angle of attack for $M_{\infty}=0.7$ and 1.2 for fin configurations $\mathrm{B}, \mathrm{C}$, and $\mathrm{D}$, respectively. The control force shown in these figures is for $\phi=0^{\circ}$ and the horizontal panels deflected $10^{\circ}$; that is, $\delta_{1}=\delta_{3}=10^{\circ}$ and $\delta_{2}=\delta_{4}=0^{\circ}$. The agreement between theory and experiment is generally fair for the three fin planforms and the angle of attack range. It can be seen from these three figures that the control force for $\alpha_{b}$ $=0^{\circ}$ for the three planforms is almost identical. Although the leading edge sweep angles are $0^{\circ}$, $38.7^{\circ}$, and $67.4^{\circ}$, respectively, all three planforms have the same aspect ratio: 1.67 . The trend with angle of attack is significantly different for the three planforms. For $\Lambda_{\ell \mathrm{e}}=0^{\circ}$ (Figure 52), the control force drops off sharply with angle of attack to the extent that for $15^{\circ}<\alpha_{b}<20^{\circ}$ a positive control input produces a negative control force. Note that this phenomenon is not caused by the body vortex wake, but by the interaction of the nonlinear normal force curve (i.e., fin stall and body upwash). For $\Lambda_{\ell \mathrm{e}}=38.7^{\circ}$ (Figure 53), $\Delta C_{z}$ is nearly constant for low $\alpha_{b}$ and then drops off with angle of attack. The experimental data for $M_{\infty}=0.7$ show a region of control force reversal 
near $\alpha_{\mathrm{b}}=17^{\circ}$. For $\Lambda_{l \mathrm{e}}=67.4^{\circ}$ (Figure 54), $\Delta \mathrm{C}_{\mathrm{z}}$ is almost constant out to $\alpha_{\mathrm{b}}=14^{\circ}$ and then shows a slight decrease with angle of attack. Also note the insensitivity of $\Delta \mathrm{C}_{\mathrm{z}}$ to Mach number. This is due to the low Mach number normal to the leading edge of the planform.

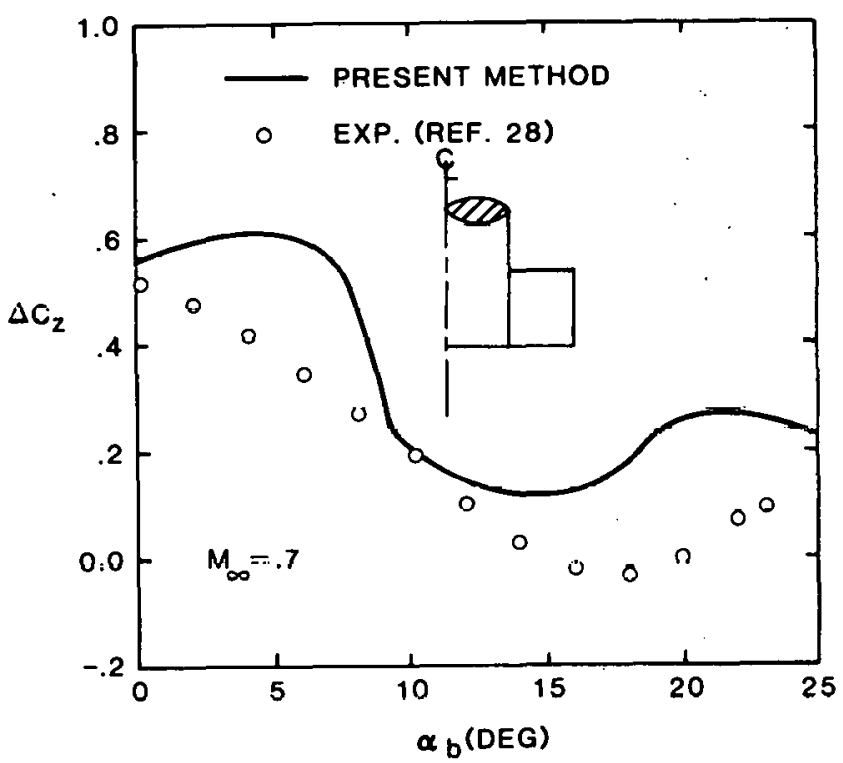

Figure 52. Pitch Control Force vs $\alpha_{\mathrm{b}}$ for Configuration $\mathrm{B}\left(\phi=0^{\circ}\right.$, $\delta_{1}=\delta_{3}=10^{\circ}, \delta_{2}=\delta_{4}=0^{\circ}$ )

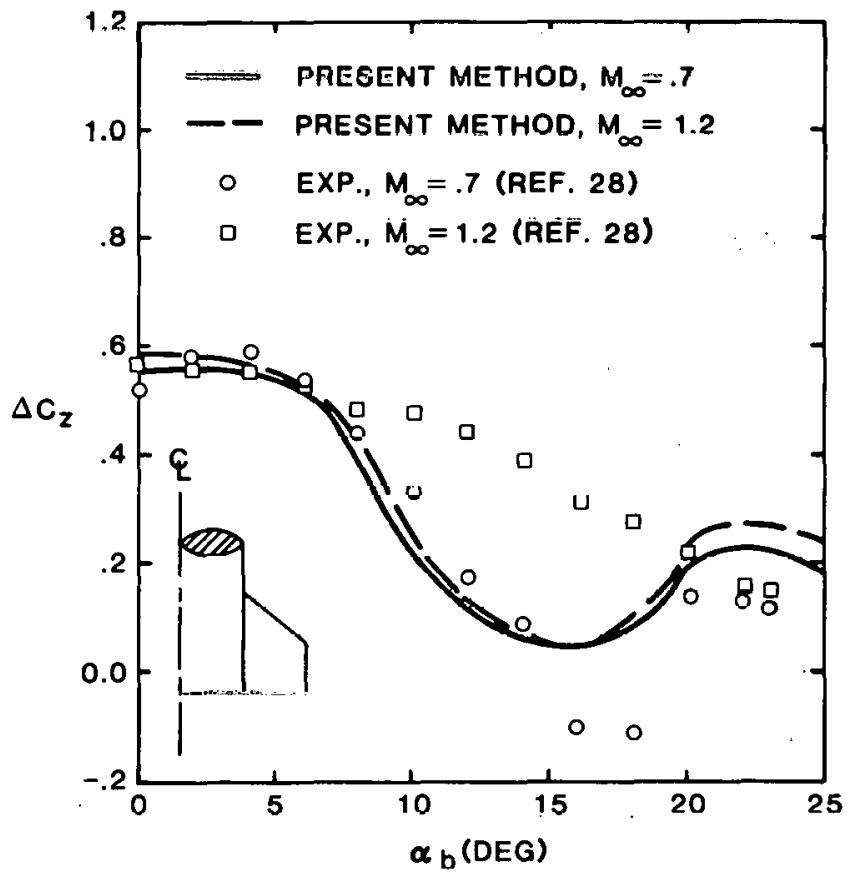

Figure 53. Pitch Control Force vs $\alpha_{\mathrm{b}}$ for Configuration $\mathrm{C}\left(\phi=0^{\circ}\right.$, $\delta_{1}=\delta_{3}=10^{\circ}, \delta_{2}=\delta_{4}=0^{\circ}$ )

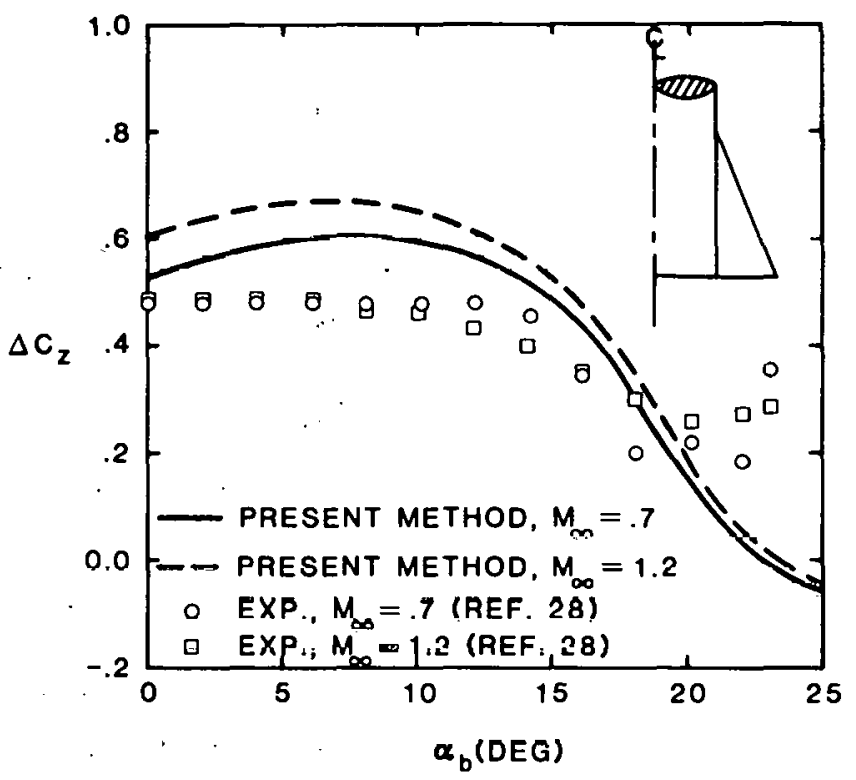

Figure 54. Pitch Control Force vs $\alpha_{\mathrm{b}}$ for Configuration $\mathrm{D}\left(\phi=0^{\circ}\right.$, $\delta_{1}=\delta_{3}=10^{\circ}, \delta_{2}=\delta_{4}=0^{\circ}$ )

Figures 55, 56, and 57 show the pitch control force vs angle of attack for all panels deflected $10^{\circ}(\phi=$ $45^{\circ}$ ) for fin planforms $B, C$, and $D$, respectively. The agreement between theory and experiment is generally fair for the three planforms over the angle of attack range. Similar trends of $\Delta C_{z}$ vs angle of attack are seen in Figures 55, 56, and 57 as compared to Figures 52, 53, and 54, respectively. For $\phi=45^{\circ}$ and $\delta$ $=10^{\circ}$, however, no control reversals are seen over the angle of attack range. Also, the delta planform (Figure 57) shows that the control effectiveness increases slightly with angle of attack up to $\alpha_{b}=12^{\circ}$ for both Mach numbers.

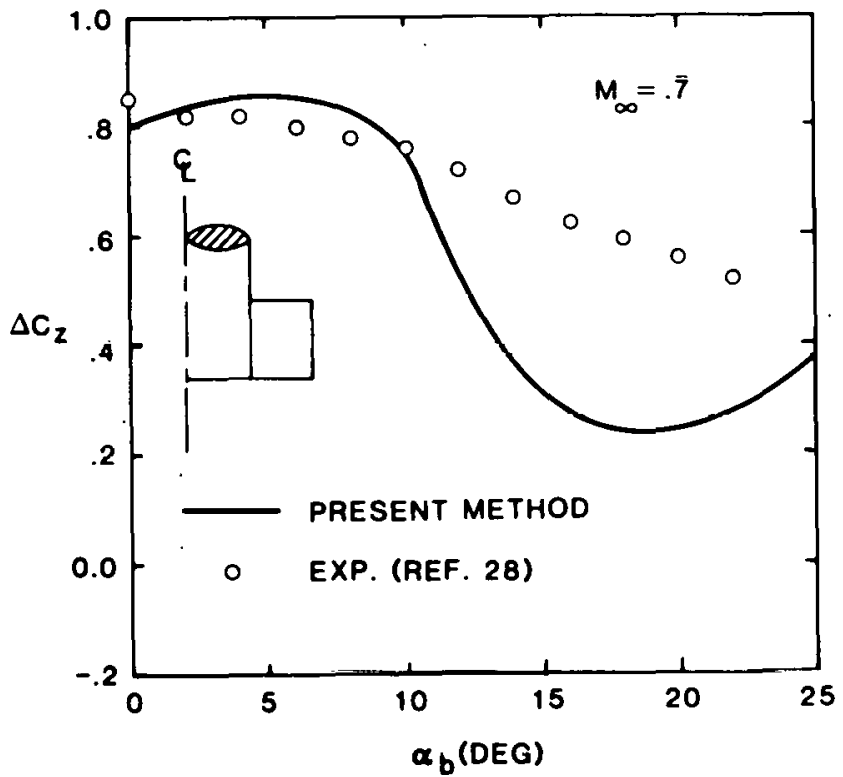

Figure 55. Pitch Control Force vs $\alpha_{\mathrm{b}}$ for Configuration B ( $\phi=$ $45^{\circ}, \delta_{1}=\delta_{2}=\delta_{3}=\delta_{4}=10^{\circ}$ ) 


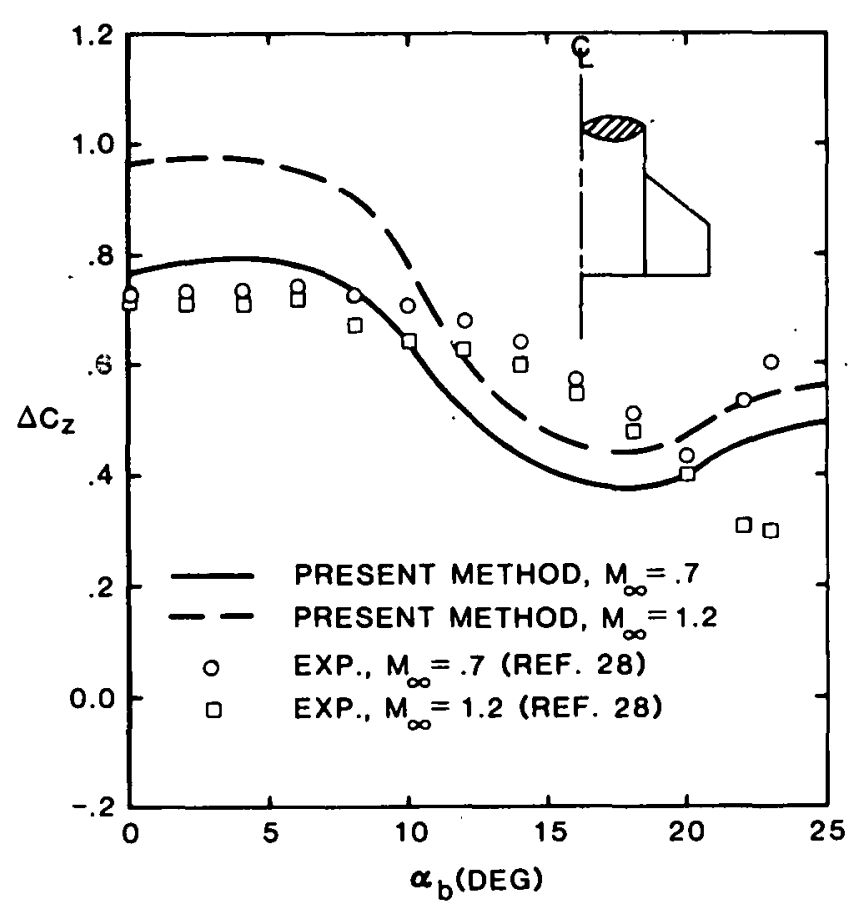

Figure 56. Pitch Control Force vs $\alpha_{\mathbf{b}}$ for Configuration C ( $\phi=$ $45^{\circ}, \delta_{1}=\delta_{2}=\delta_{3}=\delta_{4}=10^{\circ}$ )

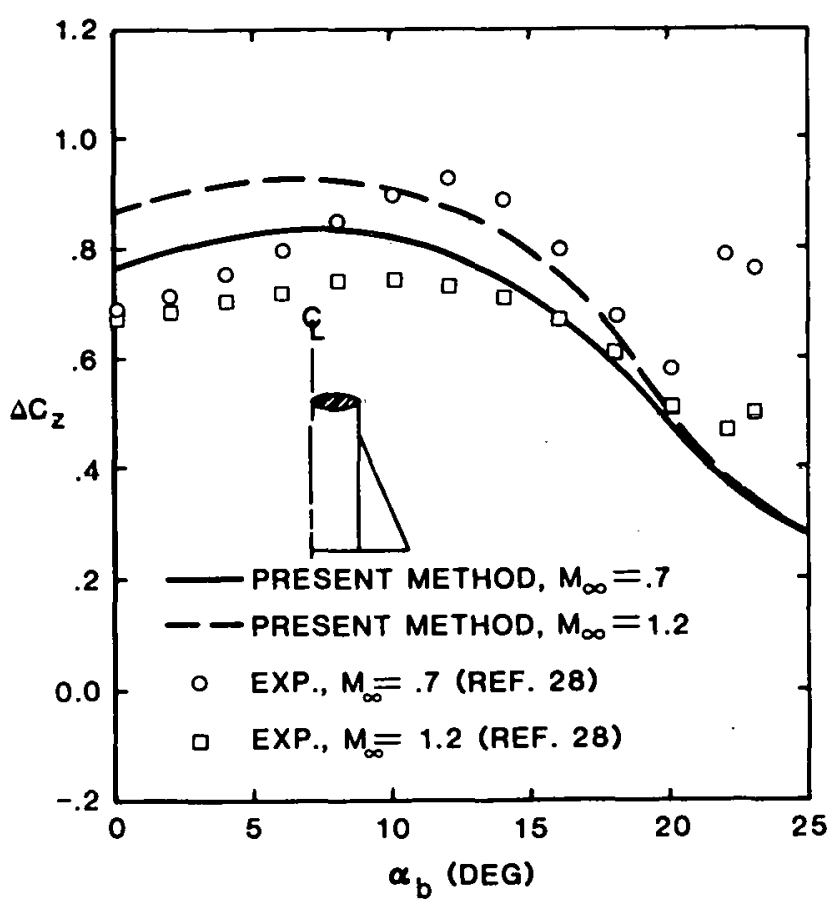

Figure 57. Pitch Control Force vs $\alpha_{\mathrm{b}}$ for Configuration $\mathrm{D}$ ( $\phi=$ $45^{\circ}, \delta_{1}=\delta_{2}=\delta_{3}=\delta_{4}=10^{\circ}$ )

\section{b. Roll Control}

Figures 58, 59, and 60 show the roll control moment vs angle of attack for $M_{\infty}=0.7$ and 1.2 for fin Configurations $B, C$, and $D$, respectively. These roll control moments are for $\phi=0^{\circ}$ and $\delta_{1}=-\delta_{3}=10^{\circ}$ and $\delta_{2}=\delta_{4}=0^{\circ}$. The theory generally predicts larger roll control moments than experiment, but the theoretical trends with angle of attack are valid. For the rectangular and clipped delta planform (Figure 58 and 59), a large region of roll control reversal occurs for $\alpha_{b}>15^{\circ}$. This region is fairly well predicted by the present analysis. For the delta planform (Figure $60)$, the theory substantially overpredicts the roll control moment up to $\alpha_{\mathrm{b}}=20^{\circ}$ and then a control reversal is predicted. The overprediction near $\alpha_{\mathrm{b}}=$ $0^{\circ}$ could be due to an inaccurate spanwise loading or it could be due to a physical characteristic of control deflection not included in the present theory (for example, root chord gap). When a fin panel is deflected for control, a spanwise gap is created at the root chord of the fin. The gap size increases as the fin deflection and root chord length increases. For Configuration $D$ the root chord length is $100 \%$ longer and $50 \%$ longer, respectively, than configurations $B$ and C. This characteristic would decrease the predicted value of the roll control moment if it were included in the theory.

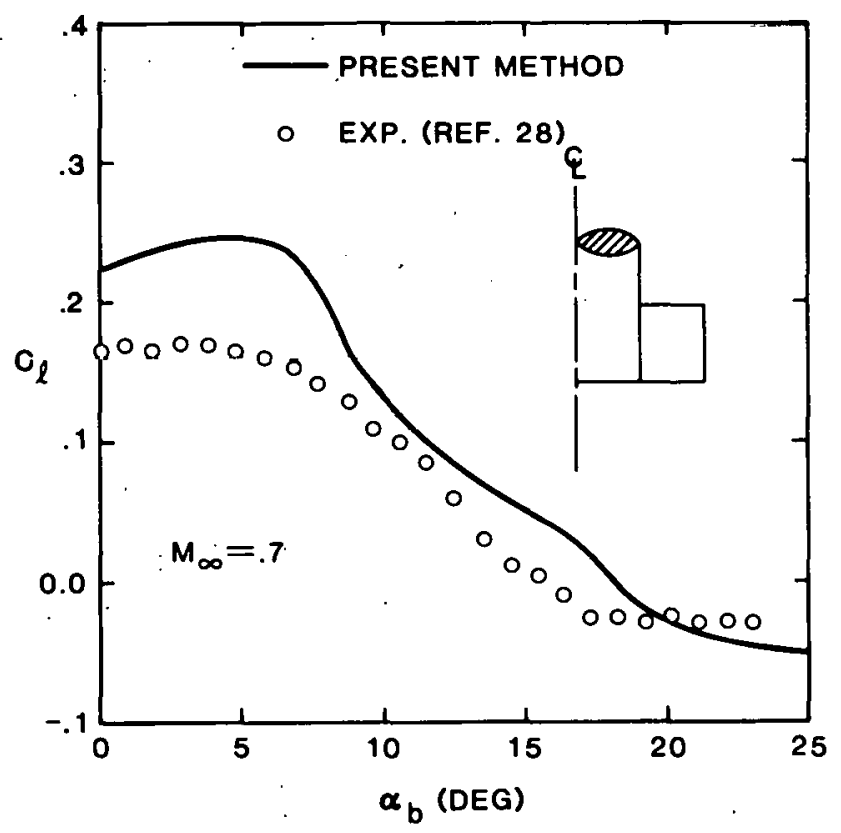

Figure 58. Roll Control Moment vs $\alpha_{\mathrm{b}}$ for Configuration B ( $\phi=$ $0^{\circ}, \delta_{1}=-\delta_{3}=10^{\circ}, \delta_{2}=. \delta_{4}=0^{\circ}$ ) 


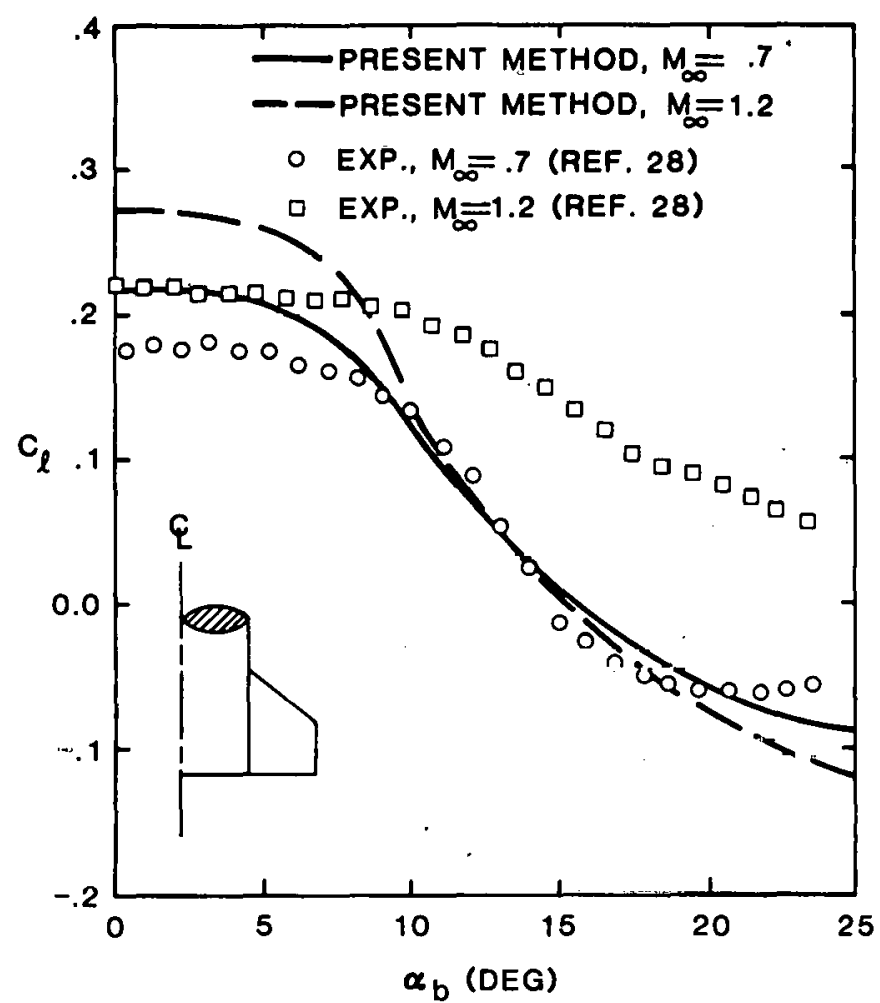

Figure 59. Roll Control Moment vs $\alpha_{\mathrm{b}}$ for Configuration C ( $\phi=$ $0^{\circ}, \delta_{1}=-\delta_{3}=10^{\circ}, \delta_{2}=\delta_{4}=0^{\circ}$ )

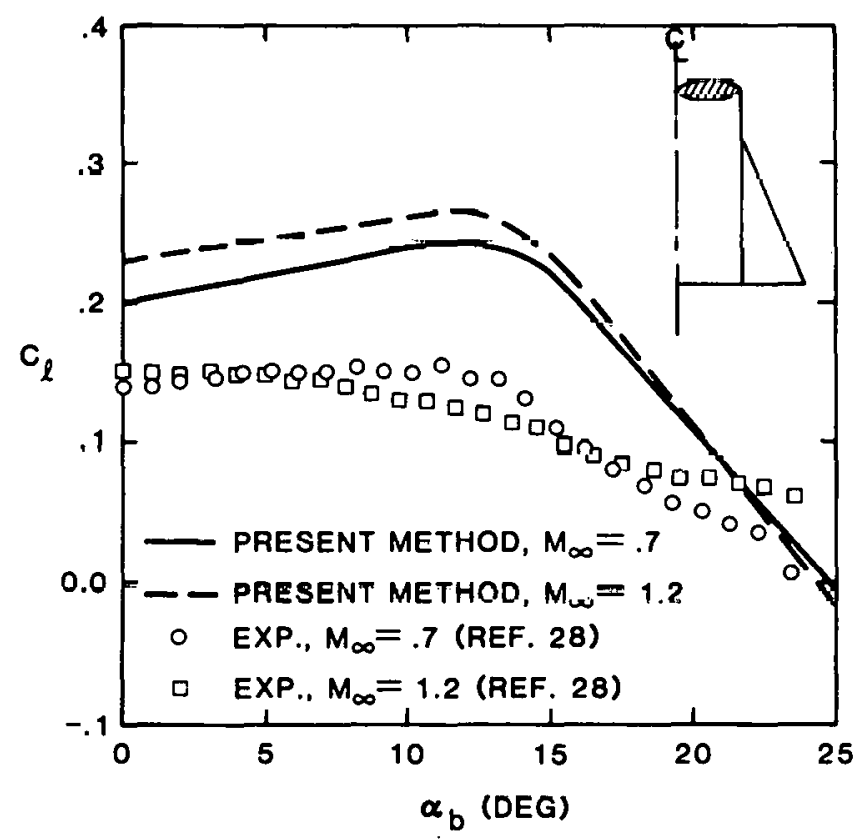

Figure 60. Roll Control Moment vs $\alpha_{\mathrm{b}}$ for Configuration $\mathrm{D}(\phi=$ $0^{\circ}, \delta_{1}--\delta_{3}=10^{\circ}, \delta_{2}=\delta_{4}=0^{\circ}$ )

\section{Conclusions and Recommenda- tions}

The following conclusions and recommendations are drawn from the present investigation.

1. The spanwise and chordwise pressure distributions are of sufficient accuracy to be used as an aid in estimating root bending and hinge moment structural loads. The hinge moment predictions can also be used as a design aid in sizing control deflection actuators.

2. Evidence was fuind lu suggest thal slall near the root chord on highly swept fins washes outboard and, consequently, decreases the outboard loading on the fin. Although this element is not included in the present theory, one might conceive of a method of approximating this phenomenon in the analysis.

3. Certain comparisons between theory and experiment suggest that the stall angle of attack increases with Mach number. If sufficient data could be gathered on this trend, it should be added to the analysis.

4. The results of the present method could be enthanced by improving the empirical data for the body flow ficld model or by using a more accurate body flow field model. An improved flow model should contain more compressibility effects.

5. In general, short span fins produce larger nonlinear forces and moments (such as induced side foree and induced roll moment) than larger span fins. This typically occurs even though the exposed fin area is smaller.

6. The present method could be improved by requiring the basic chordwise and spanwise pressure distributions to be dependent on free stream Mach number. This should be done after sufficient pressure distribution data on wings alone planforms becomes available for various Mach numbers.

7. The nonlinear decrease in roll damping for large angles of attack was found to be due to the stall angle of attack of the fin. The stall angle is primarily determined by the aspect ratio and leading edge sweep.

8. The present method should be coupled to a body force and moment predictive method 
so that complete vehicle force and moment predictions are available.

9. The rapid decrease in pitch control force at large angles of attack is predicted by the method.

10. Predicted roll control reversal at large angles of attack generally agrees with experimental data.

11. The present method should prove a valuable tool for flight vehicle designers because of its ability to address general fin planforms.

\section{References}

${ }^{1}$ J. F. Mello and K. R. Sivier, Supersonic Induced Rolling-Moment Characteristics of Cruciform Wing Body Configuration at High Angles of Attack, Aerospace Engineering, Vol 20, No. 7, July 1961, pp 20-21, 44-51.

${ }^{2}$ W. L. Oberkampf and J. D. Nicolaides, Aerodynamics of Finned Missiles at High Angle of Attack, AIAA Journal, Vol 9, No. 12, December 1971, pp 2378-2384.

3 W. L. Oberkampf, Prediction of Roll Moments on Finned Bodies in Supersonic Flow, J of Spacecraft and Rockets, Vol 12, No. 1, January 1975, pp 17-21.

${ }^{4}$ M. F. E. Dillenius and J. N. Nielsen, Prediction of Aerodynamics of Missiles at High Angles of Attack in Supersonic Flow, NEAR TR 99 (Mt. View, CA: Nielsen Enginering and Research, October 1975).

${ }^{5}$ M. R. Mendenhall and J. N. Nielsen, Effect of Symmetrical Vortex Shedding on the Longitudinal Aerodynamic Characteristics of Wing-Body-Tail Combinations, NEAR TR 69, NASA-CR-2473, (Mt. View, CA: Nielsen Engineering and Research, July 1977).

${ }^{6}$ S. B. Spangler and M. R. Mendenhall, Further Studis of Aerodynamic Loads at Spin Entry, NEAR TR 141 (Mt. View, CA: Nielsen Engineering and Research, July 1977).

${ }^{7}$ J. N. Niclsen, M. J. Hemsch, and C. $\Lambda$. Smith, A Preliminary Method for Calculating the Aerodynamic Charactristics of Cruciform Missiles to High Angles of Attack Including Effects of Roll Angle and Control Deflections, NEAR TR 152 (Mt. View, CA: Nielsen Engineering and Research, November 1977).

${ }^{8}$ F. J. Marshall and F. D. Deffenbaugh, Separated Flow Over a Body of Revolution, J of Aircraft, Vol 12, No. 2, February 1975, pp 7885

${ }^{9}$ F. D. Deffenbaugh and W. G. Koerner, Asymmetric Vortex Wake Development on Missiles at High Angles of Attack, J Spacecraft, Vol 12, No. 3, March 1977, pp 155-162.

10 W. L. Oberkampf, Aerodyanmics of Finned Missiles at High Angle of Attack, Ph.D. dissertation, August 1970, Univ. of Notre Dame, IN.

${ }^{11}$ W. L. Oberkampf and T. J. Bartel, Supersonic Flow Measurements in the Body Vortex Wake of an Ogive Nose Cylinder, AFATL-TR78-127 (Eglin AFB, FL: Air Force Armament Laboratory, November 1978).

${ }^{12}$ F. R. Grosche, Wind Tunnel Investigation of the Vortex System Near an Inclined Body of Revolution With and Without Wings, AGARDCP-71-71, Aerodynamic Interference, January 1971.

${ }^{13}$ B. E. Tinling and C. Q. Allen, An Investigation of the Normal Force and Vortex Wake Characteristics of an Ogive Nose Cylinder, NASA-TN-D-1297, April 1962.
${ }^{14}$ J. E. Fidler, J. N. Nielsen, and R. G. Schwind, An Investigation of Slender-Body Wake Vortices, AIAA 15th Aerospace Sciences Meeting, Paper No. 77-7, January 1977.

${ }^{15} \mathrm{H}$. Winter, Flow Phenomena on Plates and Airfoils of Short Span, Verein Deutscher Ingenieure, Special Issue (Avaiation) 1936 (English translation, NACA-TM-798, July, 1936).

${ }^{16} \mathrm{G}$. E. Bartlett and R. J. Vidal, Experimental Investigation of Influence of Edge Shape on the Aerodynamic Charactristics of Low Aspect Ratio Wings at Low Speeds, J Aero Sci, Vol 22, No. 8, August 1955, pp 517-533.

${ }^{17}$ D. J. Marsden, R. W. Simpson, and W. J. Rainbird, An Investigation Into the Flow Over Delta Wings at Low Speeds With Leading Edge Separation, Rept No. 114, College of Aeronautics, Cranfield, England: February 1958.

${ }^{18}$ D. Hummel, Zur Umstromung Scharfkantiger Schlander Deltaflugel bei Grossen Anstellwinkeln, Z. Flugwiss, Voi 15, No. 10, 1967.

${ }^{19}$ R. H. Wickens, The Vortex Wake and Aerod ynamic Load Distribution of Slender Rectangular Wings, Can Aeron and Sp J, Vol 13, June 1967. pp 247-260.

${ }^{20} \mathrm{R}$. Lecat and J. Rietschlin, Goniometric Aerodynamics: A Differcnt Perspective: Description-Application, AIAA Atmospheric Flight Mechanics Conference, Paper No. 79-1650, August 1979.

${ }^{21}$ E. C. Polhamus, A Concept of the Vortex Lift of Sharp-Edge Delta Wings Based on a Leading Edge Suction Analogy, NASA TN-D-3767, December 1966.

${ }^{22} \mathrm{~W} . \mathrm{H}$. Wentz and D. L. Kohlman, Wind Tunnel Investigations of Vortex Breakdown on Slender Sharp-Edged Wings, NASA-CR-98737, 1968.

${ }^{23}$ J. L. Johnson, Low-Speed Measurements of Static Stability, Damping in Yaw, and Damping in Roll of a Delta, a Swept, and an Unswept Wing for Angles of Attack from $0^{\circ}$ to $90^{\circ}$, NACA-RM-L56B01, April 1956.

24 J. N. Nielsen, Missile Aerodynamics (New York, McGraw-Hill, 1960).

${ }^{25}$ G. J. Adams and D. W. Dugan, Theoretical Damping in Roll and Rolling Moment Due to Differntial Wing Incidence for Slender Cruciform Wings and Wing-Body Combinations, NACA Report 1088, September 1950.

${ }^{26}$ J. N. Nielsen, Nonlinearities in Missile Aerodynamics, AIAA 16th Aerospace Sciences Meeting, AIAA Paper No. 78-20, January 1978.

${ }^{27}$ C. Q. Allen, R. G. Schwind, and G. N. Malcolm, Canard-BodyTail Missile Test at Angles of Attack to $50^{\circ}$ in the Ames 11-Foot Transonic Wind Tunnel, NASA-TM-78441, September 1978.

${ }^{28}$ G. E. Frantz, Control Surface Interaction Study: Wind Tunnel Test Data Analysis Report, Rept No. NA66H-139, (Columbus, OH: North American Aviation, February 1966).

${ }^{29}$ F. J. Regan, Roll Damping Moment Measurements for the Basic Finner at Subsonic and Supersonic Speeds, Rept 6652 (White Oak, MD: Naval Ordnance Laboratory, March 1964).

${ }^{30}$ L. M. Jenke, Experimental Roll-Damping Magnus, and StaticStability Characteristics of Two Slender Missile Configurations at High Angles of Attack (0 to $90 \mathrm{deg}$ ) and Mach Number 0.2 Through 2.5, AEDC-TR-58, July 1976.

${ }^{31}$ T. A. Clare, Non-Linear Resonance Instability in the Flight Dynamics of Missiles, Ph.D. Dissertation, Univ. of Notre Dame, Notre Dame, IN, June 1970.

${ }^{32} \mathrm{M}$. J. Hemsch and J. N. Nielsen, Test Report for Canard Missile Tists in Ames 6- by 6-Foot Supersonic Wind Tunnel, NEAR TR 72 (Mt. View, CA: Nielsen Engineering and Research, August 1974).

${ }^{33}$ D. E. Startzell and F. J. De Meritte, Effect of Fin Cant and Nose Shapes on Free Spin of Basic Spinner, NAVORD Rept 6025, 1958. 


\section{APPENDIX A}

\section{Structure and Listing of Computer Program}

The computer program which implements the theoretical method is constructed in a modular fashion. This design was chosen so that a user could more easily identify any difficulties one might have with the program and make changes. A large number of COMMENT cards also are included in the program to aid the user. The main program and subprogram names, card image lengths, and computer memory lengths are shown in Table A-1.

The structure of the program is depicted in the overview flow chart (Figure A-1). The flow chart shows the main program and every subprogram along with the communication between each of them. However, the flow chart does not show any data transfer by way of COMMON statements. All input and essentially all output is accomplished from the main program.

A complete listing of the computer program is given in Table A-2.

A user could rather easily reduce the computer memory requirements needed to run the program. The majority of memory $(88 \%)$ is used in two triply dimensioned arrays: VVEL $(6,73,51)$ and $\operatorname{WVEL}(6$, $73,51)$. These arrays are used to story $v$ and $w$ velocity components calculated in FLOW and transferred to FINLOAD. These arrays store $v$ and $w$ in the crossflow plane at the axial location of the fins for a given angle of attack of the body. The first subscript sets the maximum number of $\alpha_{b}$ 's that can be calculated in a given computer run, the second subscript sets the number of roll angles used to calculate fin loads, and the third subscript sets the number of spanwise locations along the exposed semispan used to calculate fin loads. By changing the program such that only three $\alpha_{b}$ 's are permitted in one run, a user can reduce computer memory by 22,338 words. The second and third subscripts should not be changed in the program for purposes of conserving memory.

Although the computer run time is discussed in Appendix B, a short discussion is given here concerning a simple modification of the code that, for certain cases, would significantly reduce the run time. The majority of computation time in the execution of the program is consumed in calculating the body flow field in subroutine FLOW. As discussed in the previous paragraph, the $v$ and $w$ velocity components are calculated in a polar grid pattern for an axial location at the fins and for a given $\alpha_{b}$. If a user plans to make a number of computer runs for fin planforms with the same semispan and located at the same axial station along the body, the modification should be made. For this case the body flow field calculated in FLOW would be exactly the same for each computer run. Therefore, the flow field stored in arrays $U$ (I), VVEL (I, J, K), and WVEL (I, J, K) should be stored on disc or tape and recalled for later computer runs. In this way the subroutine FLOW will not have to be called each time a computer run is made.

Table A-1. Description of Quantity of Computer Code

Program Element Type

Main Program

Subroutine Subprogram

Subroutine Subprogram

Function Subprogram

Subroutine Subprogram

Subroutine Subprogram

Function Subprogram

Subroutine Subprogram

Blank Common

Labcllcd Common

\section{Name Card Length}

FINLOAD $\quad 562$

IDSUB

IPSUB

CN

CNPREP

FLOW

TBLOOK

SIM

$-\cdot$

Total
22

37

25

53

134

29

26

-.

--

888

\section{Memory Length \\ (Words)}

$$
\begin{array}{r}
48349 \\
81 \\
182 \\
118 \\
302 \\
903 \\
82 \\
82 \\
16 \\
233
\end{array}
$$

50348

(142 254 ( $_{8}$ 


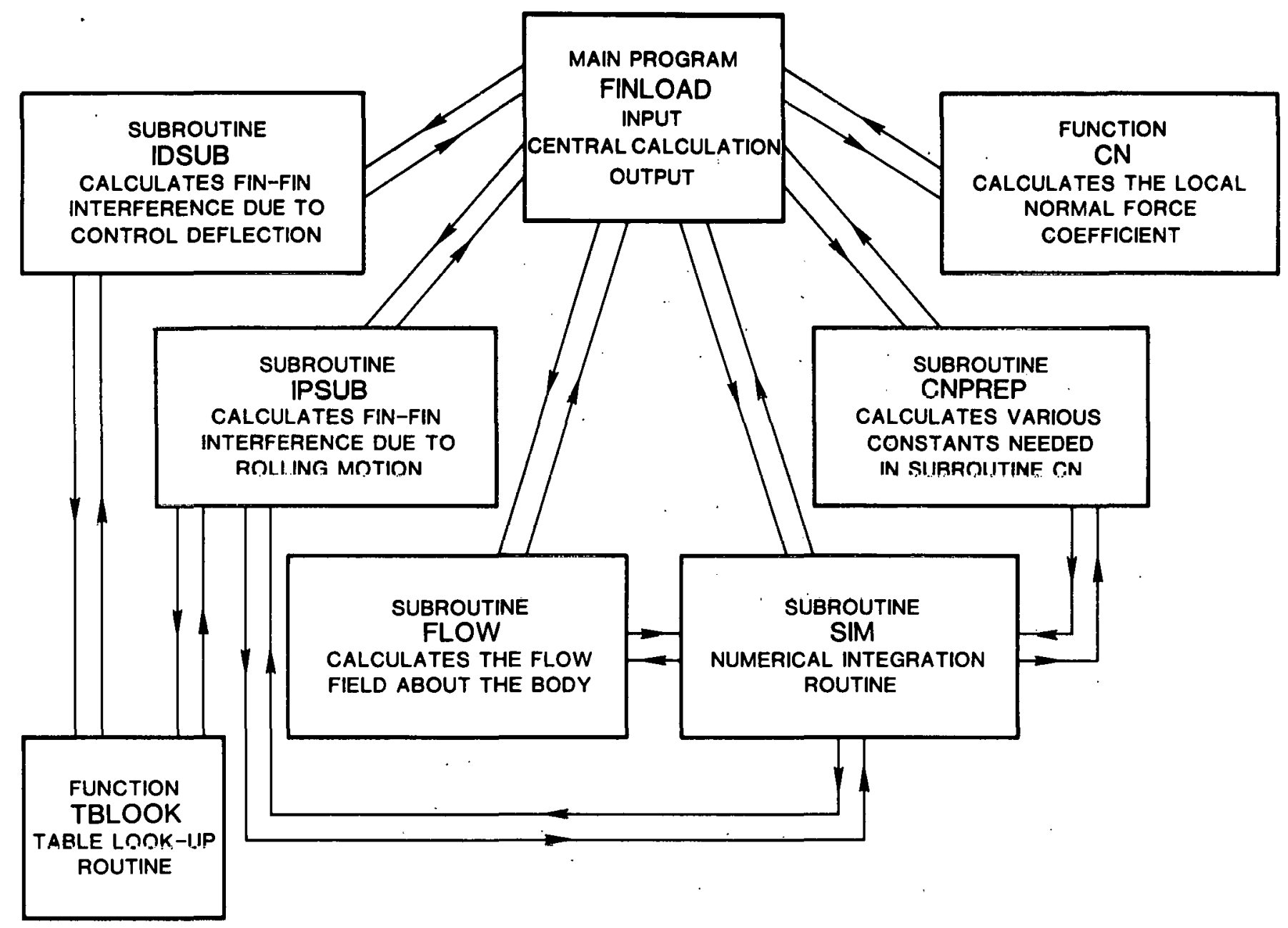

Figure A-1. Overview Flow Chart for Computer Program

Table A-2. Computer Program Listing

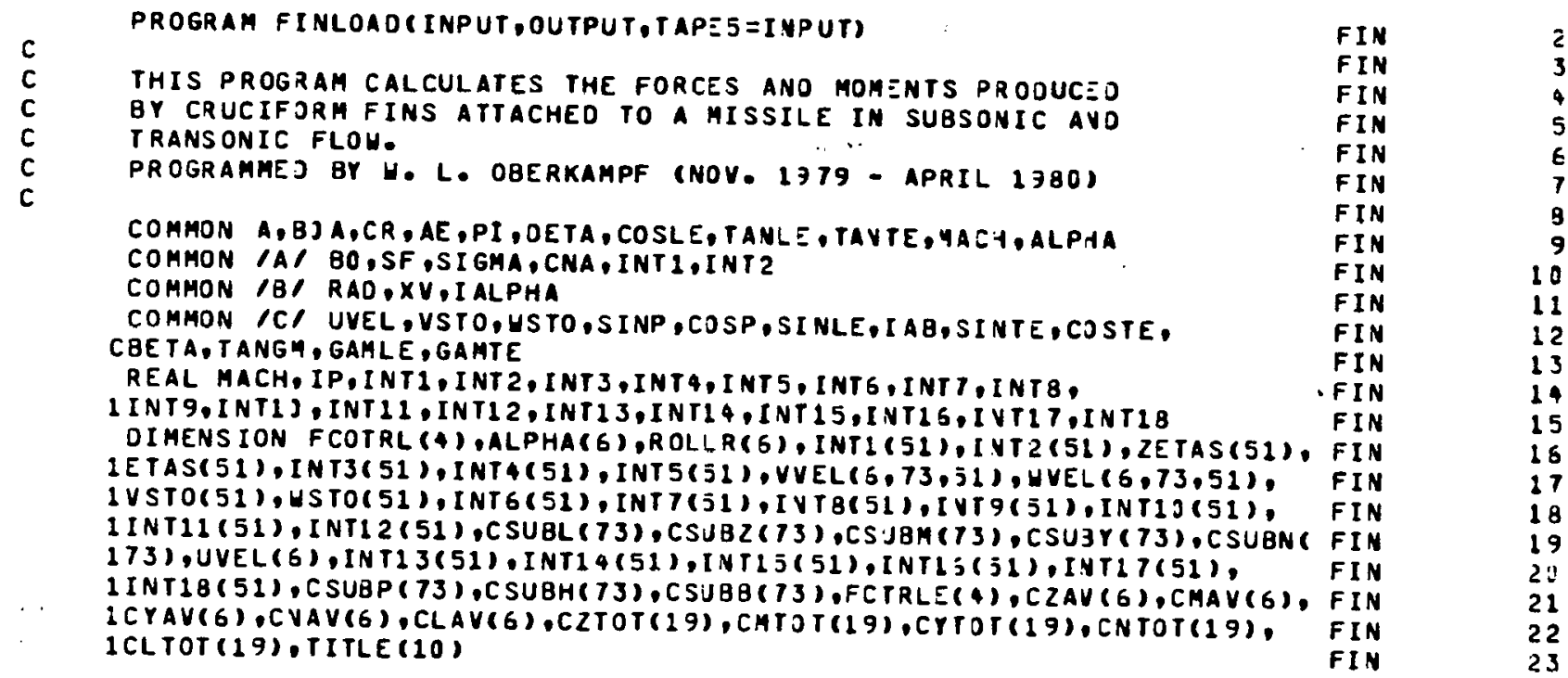


Table A-2. Computer Program Listing (Cont)

BEGIN INPUT OF MISSILE ANO FIN GEOMETRY (USE METRIC UNITSS INPUT TITLE FOR MISSILE GEOMETRY (MAXIMUH OF S) CHARACTERS)

REAO $(5,120)(T I T L E(I), I=1,10)$

$A=B O O$ Y RADIUS (METER)

BO =SEMI -SPAN (HETER)

CR $=R O O T$ OHORD (METER)

$X I=X$ LOCATION OF LEAOING EDGE ROOT CHORO (METER)

GAMLE =LEAJING EOGE SHEEP (DEG)

GAMTE = TRAILING EDGE SHEEP (OEG)

XH=X LOCATION OF PANEL HINGE MOHENT REFEREVCE

READ $(5, *)$ A, BO,CR, XI, GAMLE, GAMTE, XH

END INPUT OF MISSILE AND FIN GEOMETRY

BEGIN INPUT FOR DESIRED FORCES AND MOMENTS

ENTER I I = QUANTITY OESIRED: ENTER ZERO IF NOT JESIREJ

ICZCM $=F I N$ NORMAL FORCE AND PITCH MOMENT

ICYCN =FIN SIDE FORCE AND YAY MOMENT

ICL =FIN ROLL MOMENT

IPANEL =PANEL NORMAL FORCE, HINGE MOMENT ANJ ROOT BENOING MOMENT

READ (S,*) ICZCH,ICYCN,ICL,IPANEL

ENTER ANGULAR PARAMETERS OF FINS AND BOOY

FCOTRL (I) = CONTROL OEFLECTION OF EACH FIN (JEG); FOUR VALUES

I ALPHA =NUMBER OF BOOY ANGLES OF ATTACK (MAX. OF SIX)

ALPHA (I) =BOOY ANGLES OF ATTACK (DEG)

IROLLR = NUMBER OF BODY ROLL RATES (MAX. OF SIX)

ROLLR (I) $=80$ OY ROLL RATES (PHIDOT*B)/U, OIAENSIJVLESS)

READ (5,*) (FCOTRL $(I), I=1,4)$

READ (5,*) IALPHA, (ALPHA (I), I =1, I ALPHA)

READ (5,*) IROLLR, (ROLLR (I),I I, IROLLR)

C

ENTER FRE SSTREAM MACH NUMBER: FIN LEADING EOGE MUST BE SUBSONIC

READ $(5,4)$ HACH

END INPUT FOR FORCES AND MOMENTS

BEgIN CALEULATION DF NEEDED CONSTANTS

$P I=A C O S(-1$,

$R A D=180 . / P I$

DETA $=1 \cdot 15$ J

DZETA DETA

$A A=A \backsim A$

$B O A=B O-A$

GAMLE = GAMLE/RAO

GAMTE = GAMTE/RAD

SINLE = SIN ( GAMLE)

COSLE $=\operatorname{COS}($ GAKLE)

TANLE = TAN $($ GAMLE .

SINTE = SIN (GAMTE)

COSTE = COS (GAMTE)

TANTE = TAN ( GAMTE)

$S F=+5 \cdot B U A *+2 *(2 *-C R / B O A-T A N L E+T A N T E)$

$A E=2 * * B C A * 2 / S F$

$C T=C R-B: A=($ TANLE -TANTE)

$X V=X 1+.5 *(-25 * C R+.25 * C T+B \cap A+T A N L E)$

GAMMAM \pm ?

IF (MACH.GT.1.) GAMMAK $=A \operatorname{COS}(1, / \mathrm{MACH})$

TANGM = TAN ( GAMMAM)

IF (GAMLE.GE.GAMMAM) 60 TO 9 
Table A-2. Computer Program Listing (Cont)

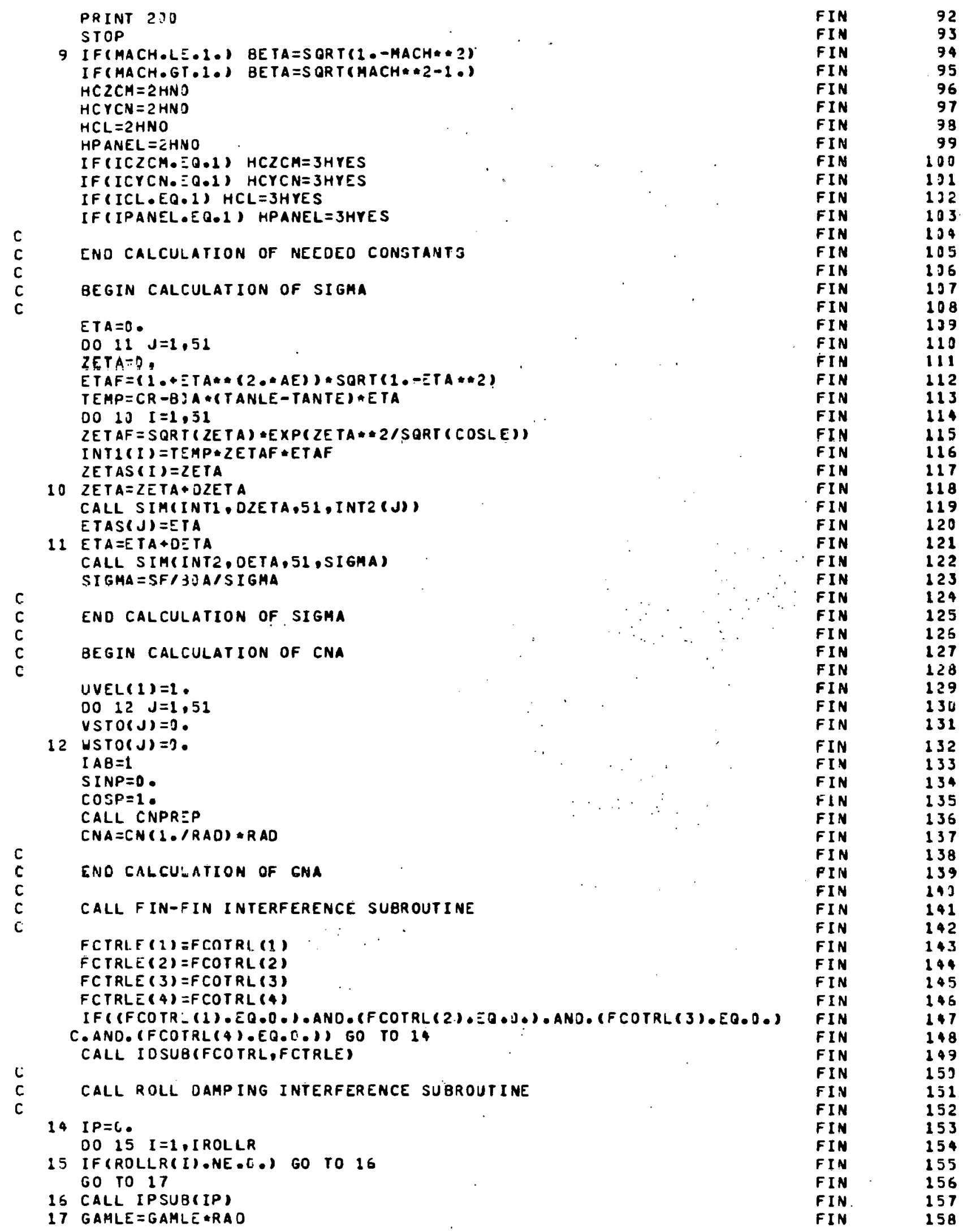


Table A-2. Computer Program Listing (Cont)

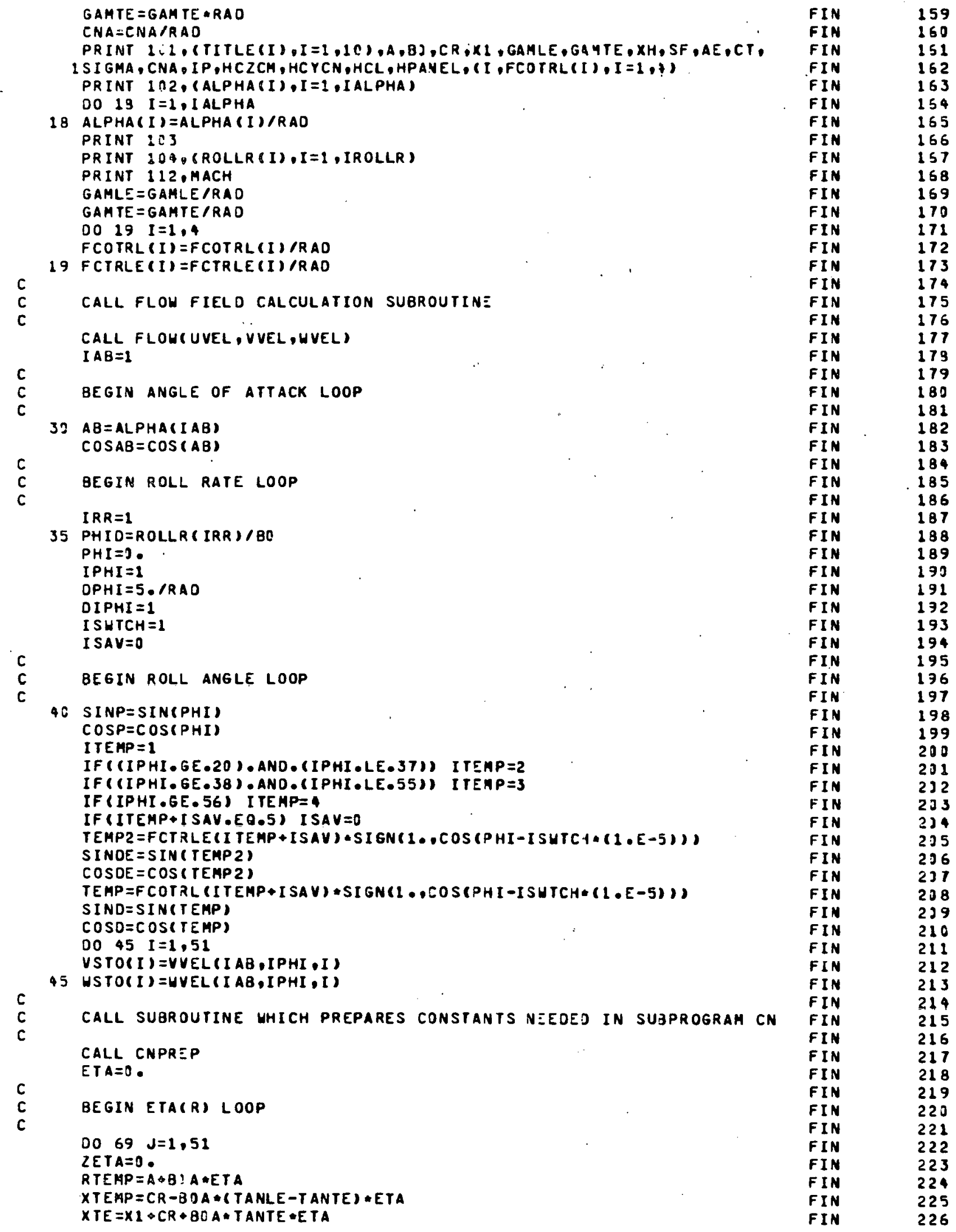


Table A-2. Computer Program Listing (Cont)

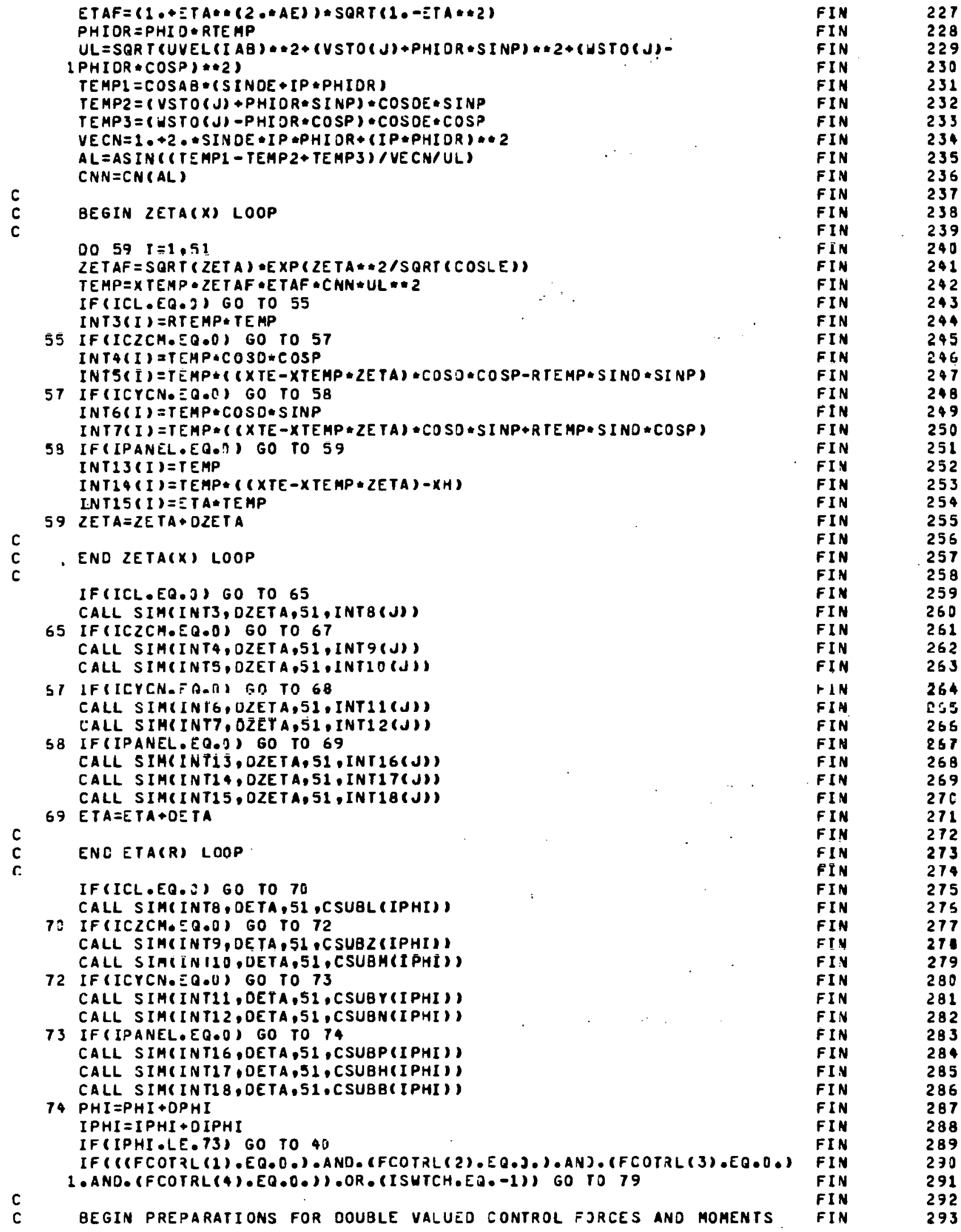


Table A-2. Computer Program Listing (Cont)

C

$P H I=P I / 2$.

I PHI $=19$

$D P H I=P I / 2$.

$D I P H I=18$

I S $U T C H=-1$

I $S A V=1$

IF (ICL.EQ.0) GO TO 75

SAVLI $9=$ CSUBL(19)

SAVL3T = CSUBL $(37)$

SAVLS5 =CSUBL (55)

75 IF (ICZCM. $E 0.0)$ GO TO 76

SAVZ1 $9=\operatorname{CSUBZ}(19)$

SAVM1 $9=$ CSUBM(19)

SAVZZT $=\operatorname{CSUBZ}(37)$

SAVM37 $=$ CSUBA 37$)$

SAVZ5 $=$ CSUBZ(55)

SAVHS5 = CSUBM(55)

76 IF (ICYCN. $E 0.0)$ GO TO 77

SAVY19=CSUBY (19)

SAVNL $9=\operatorname{CSUBN}(19)$

SAVY $37=$ CSUBY $(37)$

SAVN3 $7=\operatorname{CSUBN}(37)$

SAVY55=CSUBY (55)

SAVNS5 = CSUBN(55)

77 IF (IPANEL.EQ.\}) GO 1078

SAVP1 $9=\operatorname{CSUBP}(19)$

SAVHI $9=$ CSUBH(19)

SAVBI $9=\operatorname{CSUBB}(19)$

SAVP $37=C S U B P(37)$

SAVH3 $=$ CSU $B H(37)$

SAVBST $=$ CSUBB $(37)$

SAVPJ5 = CSUBP(55)

SAVHS5 = CSUBH( 55$)$

SAVB55 = CSUBB(55)

$78 \quad 60$ TO $4 \mathrm{U}$

$c$

$c$

ENO PREPARATIONS FOR DOUBLE VALUED CONTROL FORCES AND MOMENTS

79 CONTINUE

C

c

END OF ROLL ANGLE LOOP

BEGIN PRIMARY OUTPUT

TEMPM $=S I G M A * B O A / 2 \cdot / P I / A *+3$

TEMPF $=S I G 4 A * B O A / P I / A A$

TE MPP $=S I G M A * B O A / S F$

ATEMP $=A L P H A(I A B) * R A D$

IF (ICZCM.EQ.0) GO TO 82

$\mathrm{C}$
$\mathrm{C}$
$\mathrm{C}$

BEGIN OUTPUT FOR CZ ANO CM

PRINT 135,ROLLR(IRR), ATEMP

PRINT 105

DO $80 \quad I=1.19$

PHI $=5$. $(I-1)$

TEMPI $=T E M P F \cdot C S U B Z(I)$

TEMPZ $=$ TEMPF *CSUBZ $(I+18)$

TE MP $=$ TEMPF $: C S U B Z(I+36)$

TE $M P A=T E M P F * C S U B Z(I+54)$

IF (I.NE.19).OR.(ISWTCH.EQ.1)) SS TO 795

TEMPL = TEMPF:SAVZ19

TEMPZ = TEMPFASAVZ3T

TEMP $=$ TEMPF $\$$ SAVZS5

795 CZTOT $(1)=T E M P 1+T E M P 2+T E M P 3+T E M P 4$

8? PRINT 107,PHI, CZTOT (I ), TEMP I, TEMP 2 , TEMP $3, T$ T MP. 4

\begin{tabular}{|c|c|}
\hline $\begin{array}{l}F I N \\
F I N \\
F I N \\
F I N \\
F I N \\
F I N \\
F I N \\
F I N \\
F I N \\
F I N \\
F I N \\
F I N \\
F I N \\
F I N \\
F I N \\
F I N \\
F I N \\
F I N \\
F I N \\
F I N \\
F I N \\
F I N \\
F I N \\
F I N \\
F I N \\
F I N \\
F I N \\
F I N \\
F I N \\
F I N \\
F I N \\
F I N \\
F I N \\
F I N \\
F I N \\
F I N \\
F I N \\
F I N= \\
F I N \\
F I N \\
F I N \\
F I N \\
F I N \\
F I N \\
F I N \\
F I N \\
F I N \\
F I N \\
F I N \\
F I N \\
F I N \\
F I N \\
F I N \\
F I N \\
F I N \\
F I N \\
F I N \\
F I N \\
F I N \\
F I N \\
F I N \\
F I N \\
F I N\end{array}$ & $\begin{array}{l}294 \\
295 \\
296 \\
297 \\
298 \\
299 \\
333 \\
331 \\
302 \\
313 \\
304 \\
335 \\
336 \\
337 \\
308 \\
329 \\
310 \\
311 \\
312 \\
313 \\
314 \\
315 \\
316 \\
317 \\
318 \\
319 \\
329 \\
321 \\
322 \\
323 \\
324 \\
325 \\
326 \\
327 \\
328 \\
329 \\
3330 \\
331 \\
332 \\
333 \\
334 \\
335 \\
336 \\
337 \\
338 \\
339 \\
340 \\
341 \\
342 \\
343 \\
344 \\
345 \\
346 \\
347 \\
348 \\
349 \\
350 \\
351 \\
352 \\
353 \\
354 \\
355 \\
356 \\
357 \\
358 \\
359 \\
350\end{array}$ \\
\hline
\end{tabular}


Table A-2. Computer Program Listing (Cont)

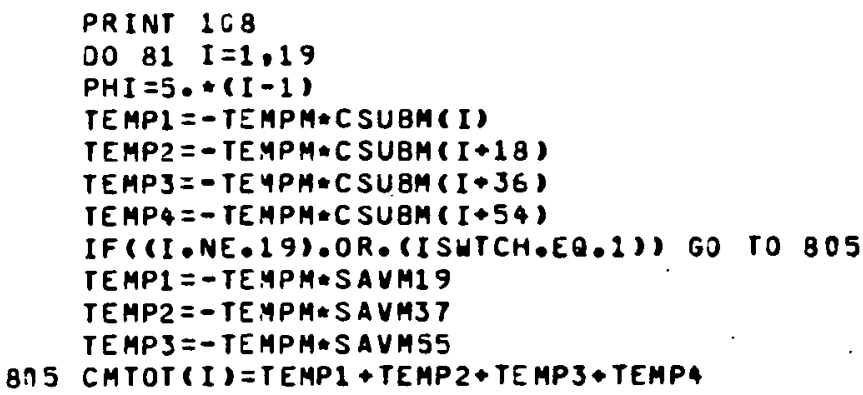

83 PRINT 147, PHI, CYTOT(I), TEMP 1, TEMP 2, TEMP3, TEMP4 PRINT 110

FIN 361

FIN 362

FIN 353

FIN $\quad 354$

FIN 365

FIN $\quad 356$

FIN 357

FIN 368

FIN 359

FIN 370

FIN. $\quad 371$

FIN $\quad 372$

FIN 373

FIN 374

FIN 375

FIN 376

FIN $\quad 377$

FIN 378

FIN 379

FIN

FIN 381

FIN 382

FIN 383

FIN $\quad 384$

FIN 385

FIN 386

FIN $\quad 387$

FIN 388

FIN 389

FIN 390

FIN 391

FIN 392

FIN 393

FIN 394

FIN 375

FIN 396

FIN 397

FIN 390

FIN 399

TIN

FIN

FIN

FIN

FIN 404

FIN 405

FIN 416

FIN 407

FIN 408

FIN 439

FIN 410

FIN 411

FIN $\quad 412$

FIN 413

FIN

FIN 415

FIN 416

FIN 417

FIN 418

FIN 419

FIN

FIN $\quad 421$

FIN $\quad 422$

FIN 423

FIN

FIN $\quad 425$

FIN $\$ 26$

FIN 427

FIN $\$ 28$ 
Table A-2. Computer Program Listing (Cont) $c$
$c$
$C$
$c$
$C$
$C$ 87 IF(IPANEL.EQ.D) GO TO 89 BEGIN OUTPUT FOR PANEL LOAOS

IF(ICL.EQ.2 9 PRINT 105,ROLLR(IRR), ATEMP PRINT 113 DO $88 \quad I=1,19$ 
Table A-2. Computer Program Listing (Cont)

PRINT 118 DO $95 I=1, I R O L L R$

95 PRINT 117,ROLLR(I),CYAV(I), CNAV(I)

96 IF (ICL.EO.J) 60 TO 99

PRINT 113

DO $97 I=1$, IROLLR c

97 PRINT 117,ROLLR (I),CLAV(I)
FIN $\quad 497$

FIN

FIN 499

FIN $53 C$

FIN FIN

FIN $5 J 2$

FIN 5 IN

FIN 534

FIN 535

FIN 536

FIN 597

END PRIMARY OUTPUT

$99 I A B=I A B+1$

IF (IAB,LE. IALPHA) GO TO 30

c

C

100 TORMAT (1H1)

101 FORAAT $11+1,11,60 X, 4 F$ I $N$ L O A D*, $11,42 X_{1}$ \#REJIETION OF FORCES AN FIN 10 MOMENTS PROOUCED BY FINS*, $/ 1,5 X, 13 A 5,1 / 5 X, 13 J$ OY RADIUS $=*$, FIN

1F6.3,* M*./15X,*FIN SEMI-SPAN =",F6.3,* M\#,//5X,*ROOT CHORD $=*$ FIN

1F.6.3,* $M *, / / 5 X, * A X I A L$ LOCATION OF FIN LEADING EOGE AT THE BOOY $=*$, FIN

IF 7.3,* M*, //5X,*LEADING EDGE SHEEP =*,FG.2,* $056 *, / / 5 X_{0}$ FIN

1. TRAILING EDGE SHEEP = "F6.2,* DEG*,//5X,

1*AXIAL LOCATION OF HINGE LINE $=*, F 7.3, * M=, 1 / 5 X$,

1. EXPOSED SURFACE AREA OF A FIN =..F7.3," SQ. $1 ., 115 \times$, 1.NORMAL FJRCE SCALING COEFFICIENT $=*, F 6.3,1 / 5 X$, *NORMAL FORCE COEFF FIN

$\bullet$ IICIENT SLOPE (FIN ALONE, N/O SFIN) = "FG०4" (L/DEG)" \#//5X, 1 - ROLL DAMPING INTERFERENCE CO FIN

IEFFICIENT $=*, F 5,3,1 / 5 X, *$ CZ AND CM OESIRED, *AL), $1 / 5 X$,

$1 * C Y$ AND CY OESIRED, $A 10, / / 5 X, * C L$ DESIRED, *A1), $/ 15 X$,

1*CP, CH AVD CB DESIRED, *A10, \$ $/ / 5 X, * C O N T Z O L$ JEFLECTION FIN*I2, FIN

1. = *,F6.2,* OEG*1, /15X,*ANGLES OF ATIACK DESIRED (DEG)*) FIN

102 FORMAT (1)X,6FT.1)

103 FORMAT ( $/ 5 X, * R O L L$ RATES DESIRED (OIMENSIONLESS) $*$ )

104 FORMAT (1) $X, 6 F 7,3)$

105 FORMAT( $2 H L, 1 / 5 X$ * *ROLL RATE $=\#, F 6.3, *$ (DIMENSIONLESS)*,5X, 1*ANGLE OF ATTACK =*.F5.1,* OEG*)

106 FORMAT $\left(1 / 1 / 5 X_{0} * P H I *, 10 X_{*} * C Z\right.$ FINS*, 1$] X_{0} * C Z$ FINL*,10X, *CZ FIN2*, $110 X, * C 2$ FIN $3 * 10 X * C Z$ FIN4**/)

107 FORMAT (T.3.1,F26.3, +F17:3)

108 FORMAT $/ 1 / 15 X_{0} * P H I *, 10 X_{0} * C H$ FINS*, $13 X_{0} * C H F I N L * 10 X_{0} * C A$ FIN2*, $110 X, * C M$ FIN 3*, $26 X_{,} * C H$ FIN4*,N)

109 FORMAT $1 / 115 X_{*} *$ PHI * $10 X_{0} * C Y$ FINS* $10 X_{*} * C Y$ FINL* $10 X_{*}$ *CY FIN2*, $110 X_{1}: C Y$ FIN3*,10X, 0 CY FIN**,/)

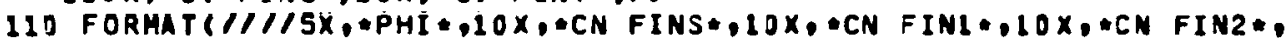
$120 X_{*}=C N$ FIN 3*,10X,*CN FIM4*,/)

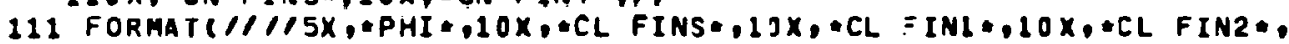
$110 X_{0} * C L$ FIN $3 *, 16 X_{0} * C L$ FIN4*,/)

112 FORHAT (IJX,AMACH NURBER $=., F 5.2)$

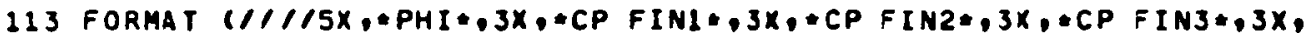

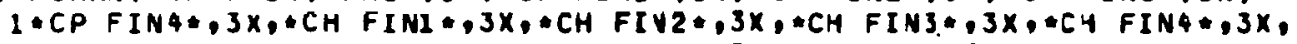
1*CB FIN $1 * 3 X, *$ CB FIN2*,3X,*CB FIN3*,3X,*CB FIN+*/I

114 FORMAT (F8.1,F9.3,11F1C.3)

115 FORMAT (1H1, $15 X$, AVERAGE VALUE OF FORCES IND YOAENTS OVER ONE ROL FIN

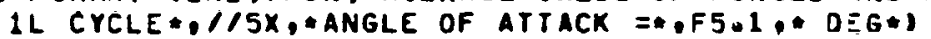

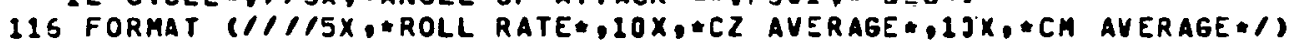

117 FORMAT (F12.3,F20.4,F2L.3)

118 FORMAT (//I5X, ROLL RATE*,10X,*CY AVERAGE*,1JX,*CN AVERAGE*/)

119 FORMAT (///5X,*ROLL RATE*,10X,*CL AVERAGE*/)

120. FORMAT (1) 196$)$

200 FORMAT (1H1,10(/),10X,

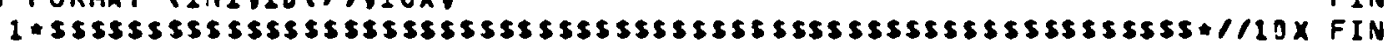
1 . MACH NUMBER TOO LARGE. SUPERSONIC LEAOING EDGE HILL RESULT.\#/110X FIN

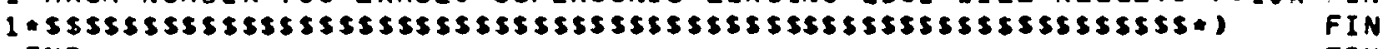
END

SUBROUTINE IOSUB(F,FE)
538

509

510

511

512

513

514

515

515

517

518

519

520

521

522

523

524

525

526

527

528

529

530

531

532

533

5.34

535

536

537

538

539

540

541

542

543

544

545

546

547

548

549

552

551

552

553

554

555

556

557

558

559

550

551

562

563

564 
Table A-2. Computer Program Listing (Cont)

$\mathrm{C}$
$\mathrm{C}$
$\mathrm{C}$
$\mathrm{C}$

THIS SUBROUTINE CALCULATES FIN-FIN INTERFERENCE JUE TO CONTROL DEFLECTIOY

COMMON A, BJA,CR,AE, PI, OETA, COSLE, TAMLE, TANTE, MAEH, ALPHA

REAL IOT, $M A C H, 10$

OIMENSION $F(4)$, I DT (11), DOBT (11),ALPHA (6), FE(4)

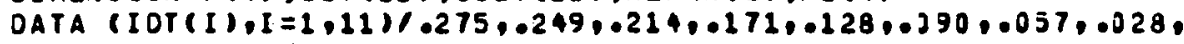

C.0CO..000.0002/

$D O B=A /(B \cap A+A)$

$x=0$.

$005 I=1.11$

OOB $(I)=x$

$5 x=x+1$

I $D=T B L O O K(D O B, D O B T, 10 T, 11)$

$F E(1)=F(1)+I D+(F(2)-F(4))$

$F E(2)=F(2)+I D *(F(2)-F(3))$

$F E(3)=F(3)+10 *(F(4)-F(2))$

$F E(4)=F(4)+I D=(F(3)-F(1))$

RE TURN

END

SUBROUTINE IPSUB(IP)

$c$
$c$
$c$
$c$

THIS SUBROUTINE CALCULATES THE ROLL DAMPING MOMENT INTERFERENCE COEFF I CIENT

COMMON A,BJA,CR,AE,PI, OETA, COSLE, TANLE, TANJE, AACH,ALPHA

COMMON /A/ BO, SF, SIGMA, CNA, INT1, INT2

REAL IP,INTI,INT2,MACH

DIMENSION DOBT (11), CLPT(11), INTL(51), , NT2(51), A. PHA(6)

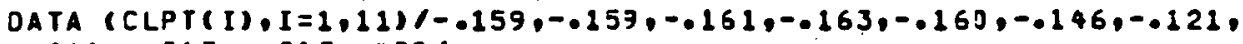

$1-.086,-.043,-.013,00001$

$S F P=2 \cdot S F+A *(2 * C R+A *(T A N L E-T A N T E))$

$A P=(2, * B D) * 2 / S F P$

$T E M P=S F P * 2 \bullet * B O /(P I * A * A * 2 \bullet * A)$

$D O B=A / B C$

$x=0$.

DO $5 I=1 . \overline{1} 1$

$00 B \operatorname{T}(I)=x$

$5 x=x+.1$

CLP $=T B L O O<(D 08, D 0 B T, C L P Y, 11)+T E A P \cdot A P$

DZETA $=1.150$.

DE TA $=D Z E T A$

ETA $=3$.

DO $11 J=1,51$

ZETA $=0$.

$0013 \quad I=1,51$

ZETAF $=S Q R T(Z E T A) \star E X P(Z E T A * * 2 / S Q R T(C O S L E))$

ETAF $=(1 \cdots E T A *(2 \cdot * A E)) * \operatorname{SORT}(1 \cdot-E T A * 2)$

$I N T 1(I)=(A+B 0 A * E T A)=2 *(C R-B O A *(T A N L E-T A N T E)=E T A)=Z E T A F * E T A F$

10 ZETA $=Z E T A+D Z E T A$

CALL SIMCINTI, OZETA.51, INT2(J))

11 ETA $=E T A+D \equiv T A$

CALL SIH(INT2, DETA.51, TE.MP)

$I P=1 \bullet+C L P /(2 * / P I * S I 6 M A * 80 A / 80 / A * \| 3 * C N A * 1 E A F)$

$I F(I P, L T \cdot\} \bullet) I P=0$.

RE TURN

END

FUNCTION CN(A)

THIS SUBPROGRAM CALCULATES THE LJCAL NORAAL FORCE COEFFICIENT GIVEN THE LOCAL ANGLE OF ATTACK

COMMON $10 /$ KP,KV,AS,SAVI,SAVZ, SAV 3

REAL KP,KV

$C N=C$.

IF (A.EQ.).) RE TURN

$\triangle S A V E=A$

$\begin{array}{ll}\text { FIN } & 555 \\ \text { FIN } & 566 \\ \text { FIN } & 557 \\ \text { FIN } & 568 \\ \text { FIN } & 569 \\ \text { FIN } & 570 \\ \text { FIN } & 571 \\ \text { FIN } & 572 \\ \text { FIN } & 573 \\ \text { FIN } & 574 \\ \text { FIN } & 575 \\ \text { FIN } & 576 \\ \text { FIN } & 577 \\ \text { FIN } & 578 \\ \text { FIN } & 579 \\ \text { FIN } & 580 \\ \text { FIN } & 531 \\ \text { FIN } & 582 \\ \text { FIN } & 583 \\ \text { FIN } & 584 \\ \text { FIN } & 585 \\ \text { FIN } & 596 \\ \text { FIN } & 587 \\ \text { FIN } & 588 \\ \text { FIN } & 589 \\ \text { FIN } & 590 \\ \text { FIN } & 591 \\ \text { FIN } & 592 \\ \text { FIN } & 593 \\ \text { FIN } & 594 \\ \text { FIN } & 535 \\ \text { FIN } & 596 \\ \text { FIN } & 597 \\ \text { FIN } & 598 \\ \text { FIN } & 599 \\ \text { FIN } & 630 \\ \text { FIN } & 631 \\ \text { FIN } & 602 \\ \text { FIN } & 633 \\ \text { FIN } & 634 \\ \text { FIN } & 635 \\ \text { FIN } & 636 \\ \text { FIN } & 637 \\ \text { FIN } & 638 \\ \text { FIN } & 639 \\ \text { FIN } & 610 \\ \text { FIN } & 511 \\ \text { FIN } & 612 \\ \text { FIN } & 613 \\ \text { FIN } & 514 \\ \text { FIN } & 615 \\ \text { FIN } & 616 \\ \text { FIN } & 617 \\ \text { FIN } & 618 \\ \text { FIN } & 519 \\ \text { FIN } & 620 \\ \text { FIN } & 621 \\ \text { FIN } & 622 \\ \text { FIN } & 623 \\ \text { FIN } & 624 \\ \text { FIN } & 625 \\ \text { FIN } & 626 \\ \text { FIN } & 527 \\ \text { FIN } & 628 \\ \text { FIN } & 629 \\ \text { FIN } & 630 \\ \text { FIN } & 631 \\ \text { FIN } & 632 \\ \text { FN } & \\ \text { FN } & 51 N\end{array}$


Table A-2. Computer Program Listing (Cont)

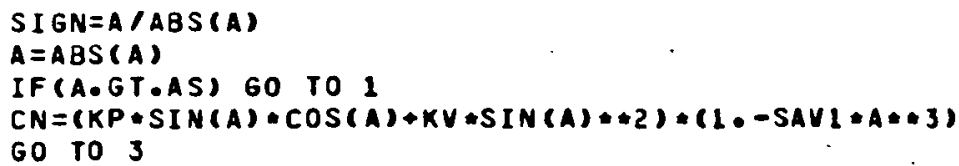

1 CNAS $=(K P * S I N(A S) * \operatorname{COS}(A S)+K V * S I N(A S) * 2) *(1,-S A V I * A S * 3)$

IF (A.GT.2.:AS) GO TO 2

CNAMAS $=(K P \bullet S I N(A-A S) \bullet \operatorname{COS}(A-A S)+K V * \operatorname{SIN}(A-A S) * 2) *(1-S A V I *(A-A S) * 3)$ $C N=S A V 2 * C Y A S+S A Y 3 * C N A M A S$ GO TO 3

$2 C N=(S A V 2+S A V 3) \cdot C N A S$

$3 C N=S I G N \cdot C N$

$A=A S A V E$

RE TURN

ENO

SUBROUTINE CNPREP

THIS SUBPROGRAH CALCULATES VARIOUS CONSTANTS MEEDED IN THE SUBPROGRAM CN

COAMON A, JOA,CR,AE, RI, OETA, COSLE, TANLE, TANTE, HACIT, ALFIIA COMMON /C, UVEL, VSTO, USTO, SINP, COSP, SINLE, IAB, SINTE, COSTE, CBETA, TANGH, GAHLE, GAHTE

COMMON $/ D /$ KP,KV,ASTL,SAVI, SAV2,SAV3

REAL MACH, MU,KP,KV

OIMENS ION VSTO(51), USTO(51), UVEL (6), ALPHA(5)

UAV =UVEL ( I AB)

CALL SIM(VSTO, DETA, 51 , VAV)

CALL SIMC HSTO, DETA, 51 , WAVI

VRAV $=V A V+C O S P+H A V+S I N P$

GAMLEE = GAHLE+ASIN(VRAV/SORT $(U A Y * * 2+V R A V * * 2))$

GAMTEE = GAMTE+ASIN(YRAV/SORT (UAY $* 2+V R A V * 2)$ )

IF (ABS (GAMLEE) . OT.1.57) GAMLEE =S[ GN (1.57,6AMLEE)

IF (GAMLEE •LT•0 O) GAMLEE =0.

IF (AB S (GAMTEE) . GT.1.57) GAMTEE =SI GN(1.57, GAMTEE)

COSLEE = COS (GAMLEE)

SINLEE = SIN ( GAMLEE)

TANTEE = TAY (GAMTEE)

$C R E=C R * C O S T E / C O S(G A M T E-B A M L E+B A M L E E)$

$B D A E=B O A \div C O \& L E E / C O S L E$

$A E E=4, /(2 . * C R * \operatorname{COSTE} * \operatorname{COSLE} /(80 A * C O S L E E * \operatorname{COS}(G A M T E-G A M L E+G A M L E E))$ -

ITANTEE-TAY (GAMLEE)

CRS $=C R E+B J A E+T A N T E E$

GAMMA = A TAY (CRS/BCAE)

TANG = TAN(SAMMA)

$P S=2, / A E E / T A N G$

IF (MACH.GT.1.) PS=PS/(1.-TANGH/2./TANG)

PSI =AT AN (2, $A P S * T A N G)$

IF(MACH.GT.1.) PSI=ATAN(2.*PS*(TANG-TANGH))

TANPSI = TAN (PSI)

SINPSI = SIN (PSI)

$K P=4: * P I /(T$ ANP SI $+S Q R T(T A N P S I * 2+S I N P S I * * 2 I P S *+2+4 * * B E T A * * 2))$

$K V=(K P-K P * 2 * T$ ANPSI $/ 4 \ldots / P I) \div S Q R T(1++T A N P S I \cdots 2)$

$A E T E M P=A E S$

IF ( AETEMP•LT.1 •) AETEMP =1 .

IF (AETEMP. 6T.4.) AE TEMP $=4$.

ASTL $=.6632 *(1 . * .02 *(A E T E M P-2) * 4) *,(1 *+1 * 1 *(A E T E M P-1 *) * 2 * 5 *$

SAVI $=(1 \cdot-$ MU) /ASTL*A3

SAV2 $=1--035 *(1-+E X P(A E T E M P) / 80 * S I N L E E * *(1 . / A E T E M P) * S I V($ (AETEMP-2.1 FIN

C) GAMLEE) $) /(1+\ldots 5 *(A E T E M P-1 \cdot 1 * 13)$

IF (SAV2 -LT..65) SAY $2=.65$

SAV3 = COSLEE *2*AEE 110 .

RE TURN

END 
Table A-2. Computer Program Listing (Cont)

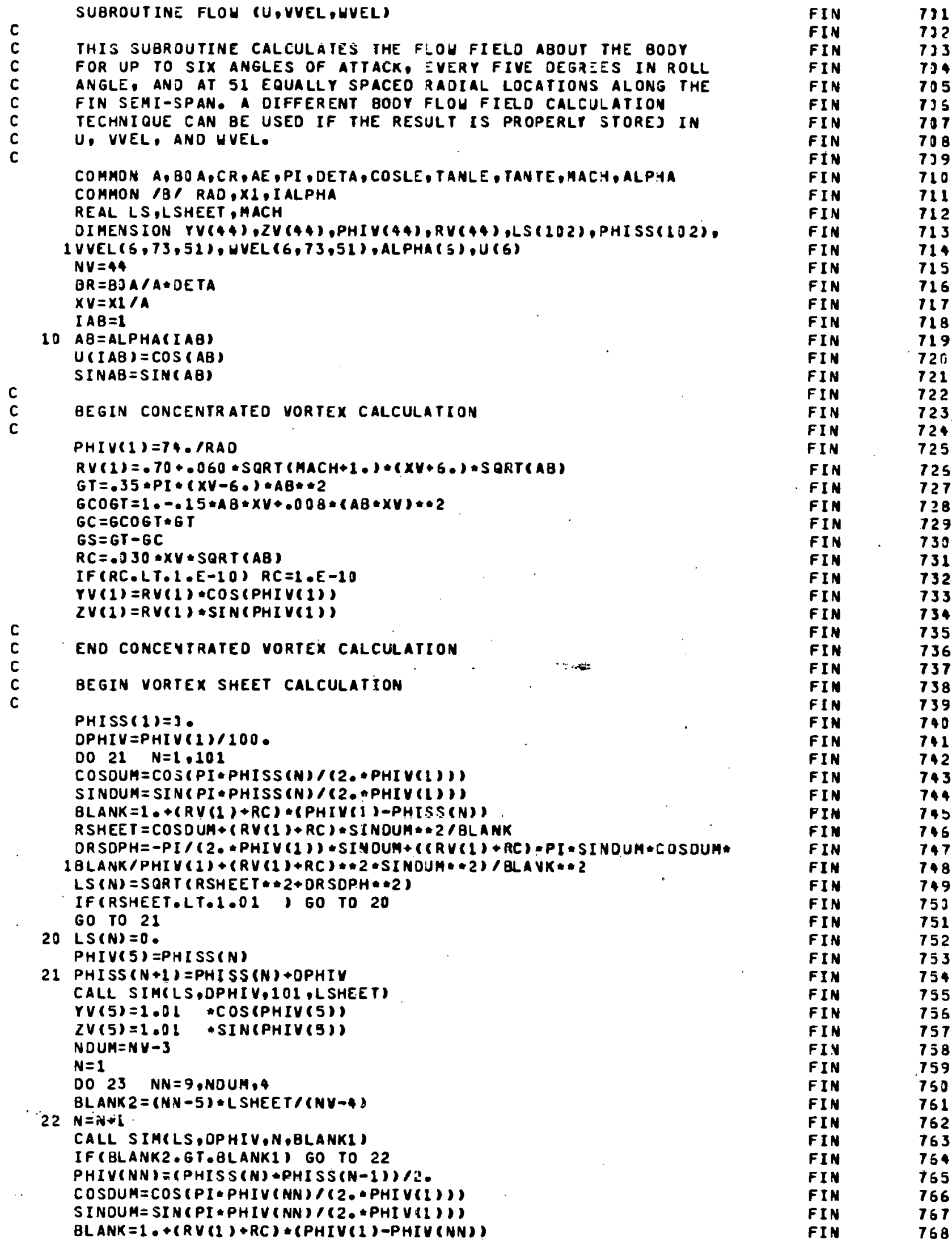


Table A-2. Computer Program Listing (Cont)

$R V(N N)=\operatorname{COSOUM}+(R V(1)+R C) * S I N O U M+12 / 8 L A M K$

$Y V(N N)=R V(N N)+\operatorname{COS}(P H I V(N N))$

$232 V(N N)=R V(N N) \cap S I N(P H I V(N N))$

NOUR=NV -2

$0024 \quad N=2, N D U M, 4$

$Y V(N)=\quad Y V(N-1) /(Y V(N-1) * 2+2 V(N-1) *+2)$

$Z V(N)=\quad Z V(N-1) /(V V(N-1) * 2+2 V V(N-1) * 22)$

$Y V(N+1)=-Y V(N)$

$2 V(N+1)=2 V(N)$

$Y V(N+2)=-Y V(N-1)$

$242 V(N+2)=2 V(N-1)$

$c$

C

ENO VORTEX SHEET CALCULATION

BEGIN VELOCITY COMPONENT CALCULATION

PHI =0.

$I P H I=1$

3] $P H I=P H I / R A O$

$\operatorname{COSP}=\operatorname{COS}(P H I)$

3 I NPI 3 I A ( PIII)

$R=1$.

I $R=1$

$31 \quad r=R * \operatorname{COSP}$

$Z \equiv R \cdot S I N P$

IF (ABS $(Y-Y Y(1)) \cdot L T \cdot A B S(Y-Y Y(4))) 60$ TO 32

$Y 14=Y V(4)$

$214=Z V(4)$

GO TO 33

$32 \quad Y 14=Y V(1)$

$214=2 V(i)$

$33 C=1.254 *((Y-Y 14) * * 2+(Z-Z 14) * * 2) / R C * 2$

$v=\mathbf{C}$.

$H=0$.

$0034 \mathrm{~J}=1.4$

$\operatorname{CON} 1=(r-1 V(J)) * 2+(Z-Z V R J)) n+2$

$v=v+(-1) * J *(Z-Z v(J)) / C O N 1$

$34 H=W+(-1) * * J *(Y-Y V(J)) / C O N 1$

VS $=0$.

$\forall S=0$,

DO $35 N=5, N V$

CONL $=(Y-Y V(N)) * 2+(Z-Z V(N)) * 2$

$V S=V S+(-1) * N *(Z-Z V(N)) / C O N I$

$35 W S=W S+(-1) * \cdots N *(Y-Y V(N)) / C O N 1$

$V=$

$(-2 * \quad Y * Z * S I N A B /(Y * * 2+Z * * 2) * 2 * 2+3 E * V /(2 * P I) *$

$12 \cdot * G S /(P I *(N V-4)) \cdot V S)+(1 \cdot-E X P(-C))$

$H=\quad$ (SINAB*(1** $\quad(Y * * 2-Z * * 2)(Y(Y * * 2+Z * * 2) * 2)-$

$1 G C * H /(2 * P[)-2 * G S * H S /(P[*(N V-4))) *(1,-E X P(-C))$

IF (ABS $V), G T=2, Y=20 * Y / A B S(V)$

IF (ABS (W).6T.2.) H=2.*H/ABS(H)

VVEL(IAB,IPHI,IR) $=V$

HVELII $I A B, I P H I, I R)=4$

$R=R+R R$

$I R=I R+1$

IF(IR.LE.5I) GO TO 31

$P H I=P H I * R A O$

$P H I=P H I+5$.

$I P H I=I P H I+1$

IFIIPHI.LE.73) 60 TO 30

$I A B=I A B+1$

$c$
$c$

IF (IAB.LE. IALPHA) GO TO 10

END VELOCITY COMPONENT CALCULATIJN

RETURN

END

C

FUNCTION TBLOOK(X,AB,ORD,N)

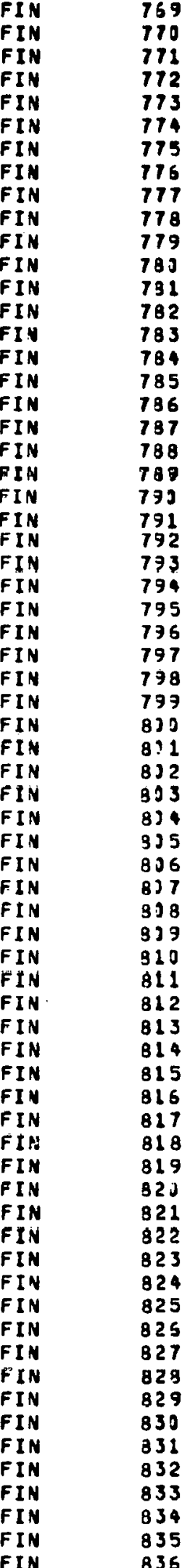


Table A-2. Computer Program Listing (Cont)

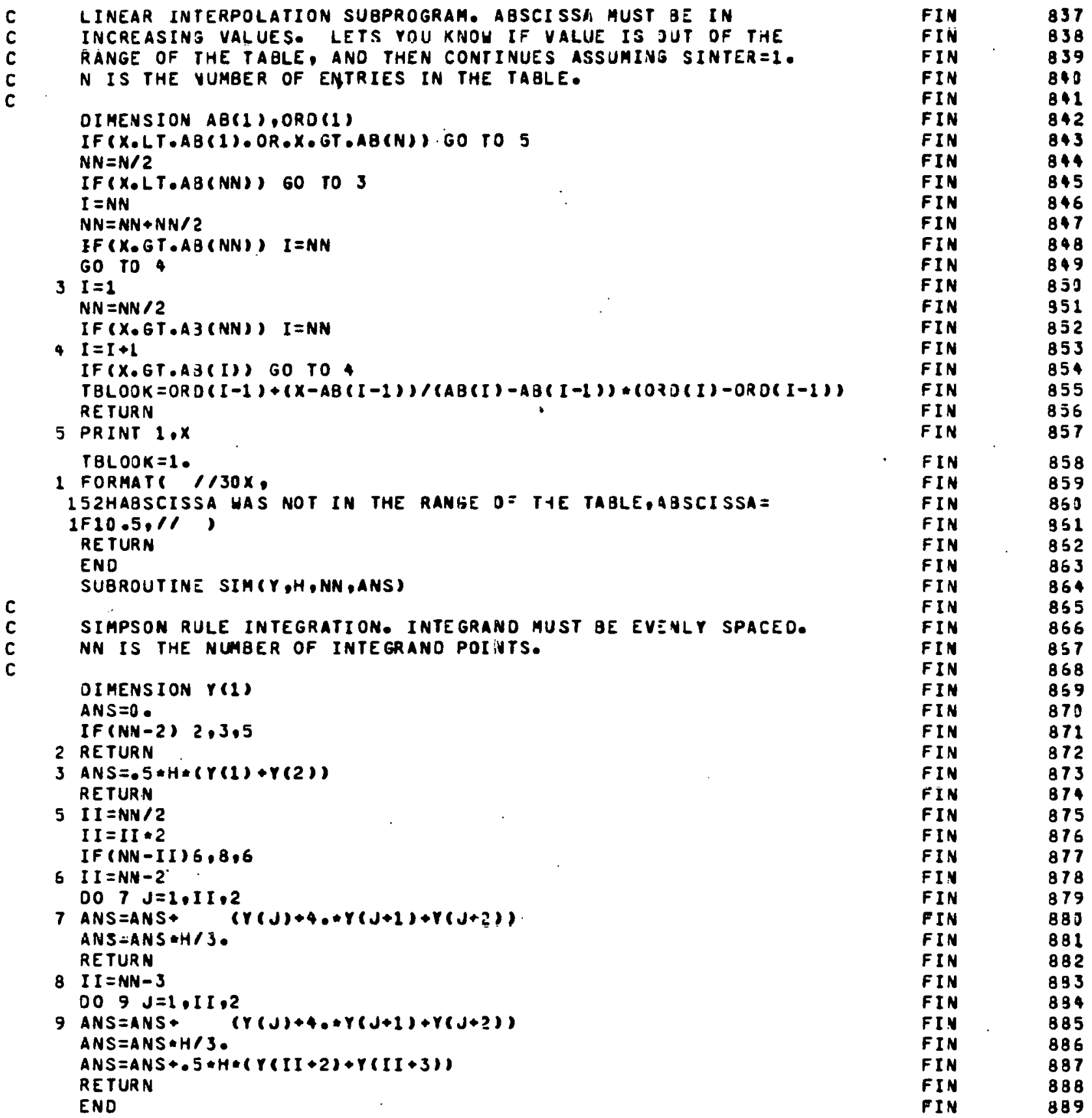




\section{APPENDIX B}

\section{Use of the Computer Program}

The input for the program is very simple. Seven data cards are needed to execute the program.

Card 1: Input the title of the missile and fin geometry. The title can be any combination of letters and numbers and must be located in columns 1 through 60 . Sample card:

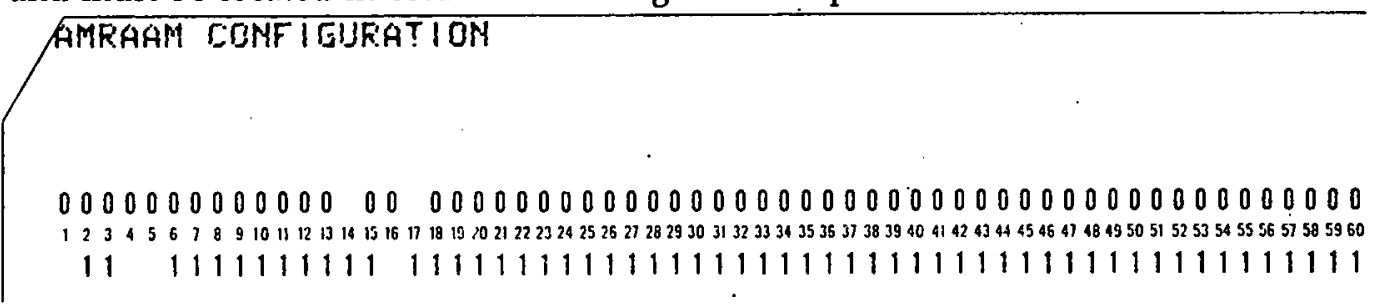

Card 2: Input missile and fin geometry. The order of input variables is: body radius (meter), fin semispan (meter), fin root chord (meter), axial location of where the fin leading edge intersects the body (meter), fin leading edge sweep (degree), fin trailing edge sweep (degree), and axial location of panel hinge moment reference (meter). All data (cards 2 through 7 ) are input in free format; that is, any format you like. Sample card:

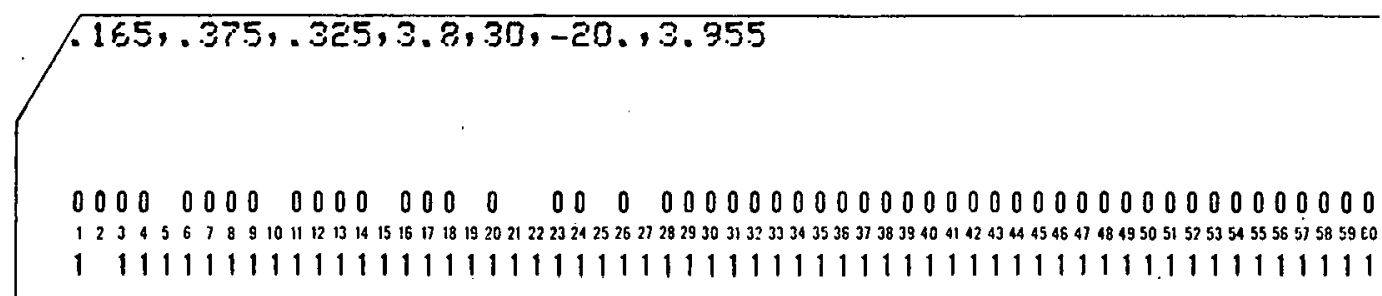

Card 3: Input the forces and moments that the user desires. Enter 1 if quantity is desired and enter 0 if quantity is not desired. The order of input variables is: fin normal force and pitch moment, fin side force and yaw moment, fin roll moment, panel normal force, hinge moment, and root bending moment. Sample card:

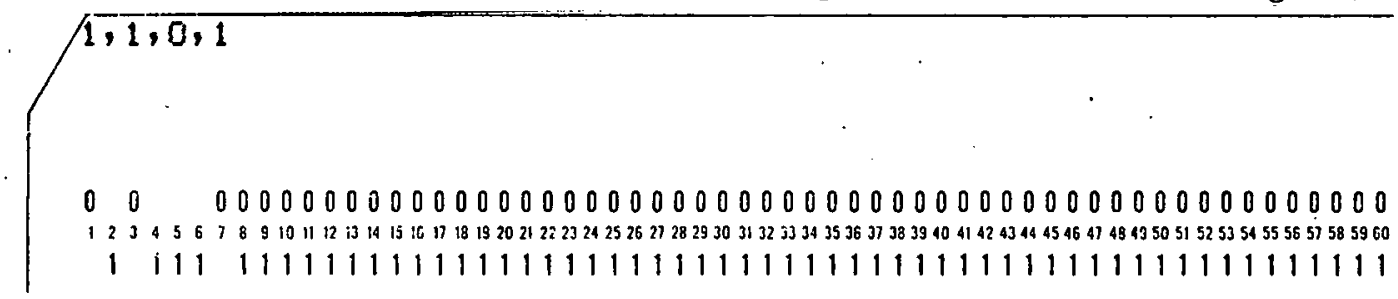

Card 4: Input the control deflection angle of each fin. The order of input variables is: control deflection Fin 1 (degree), cuntrol deflection Fin 2 (degree), control deflection Fin 3 (degree), and control deflection Fin 4 (degree). Use the sign convention shown in Figure 10 of this document. Sample card.

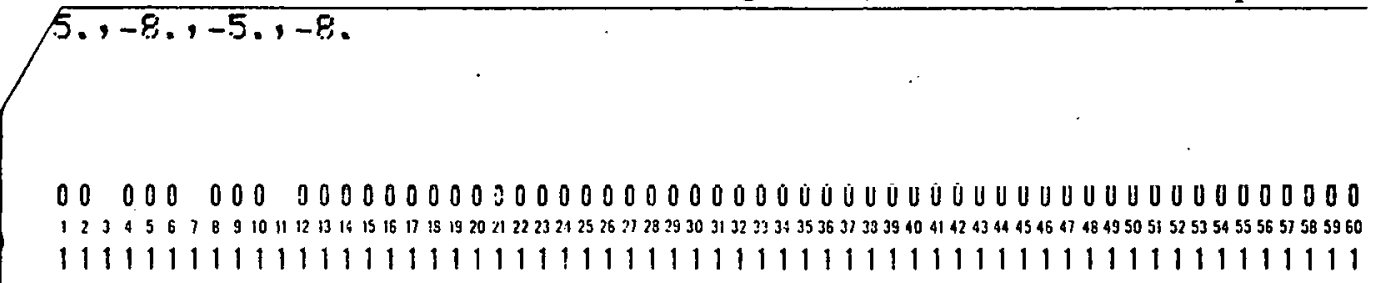


Card 5: Input the number of angles of attack desired and the angles of attack of the body desired. The maximum number of angles of attack allowed is six. The order of input variables is: number of body angles of attack desired, first angle of attack of the body desired (degree), second angle of attack of the body desired (degree), third angle of attack of the body desired (degree), fourth angle of attack of the body desired (degree), fifth angle of attack of the body desired (degree), and sixth angle of attack of the body desired (degree). Sample card:

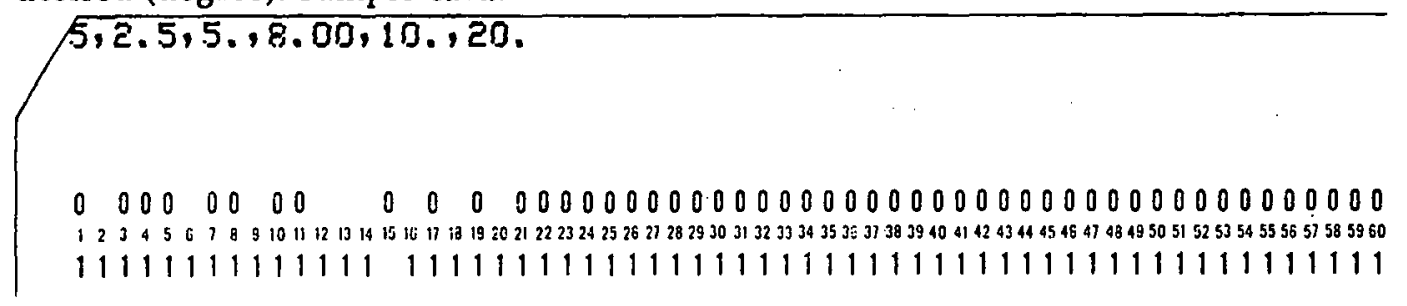

Card 6: Input the number of roll rates desired and the specific roll rates desired. The maximum number of roll rates allowed is six. The input roll rate must be nondimensionlized as $\dot{\phi} b_{0} / U_{\infty}$. The order of input variables is: number of roll rates desired, first roll rate desired, second roll rate desired, third roll rate desired, fourth roll rate desired, fifth roll rate desired, and sixth roll rate desired. Sample card:

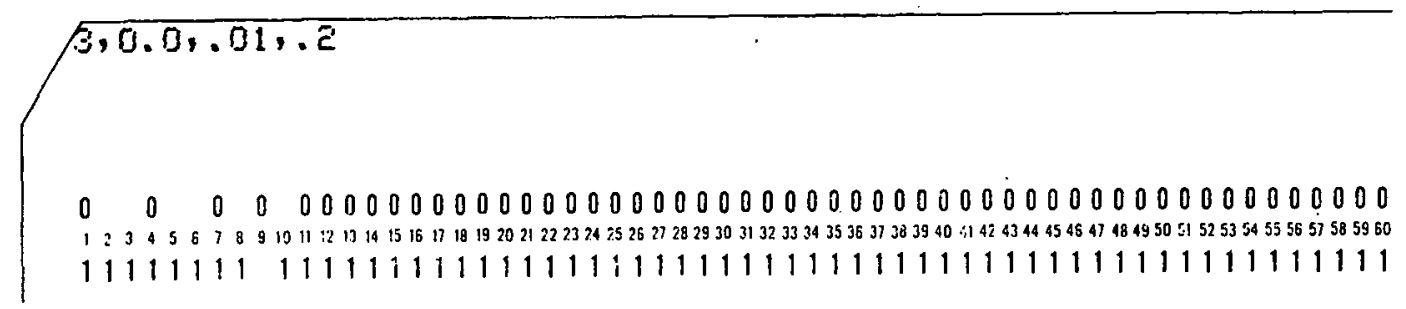

Card 7: Input the free stream Mach number. Sample card:

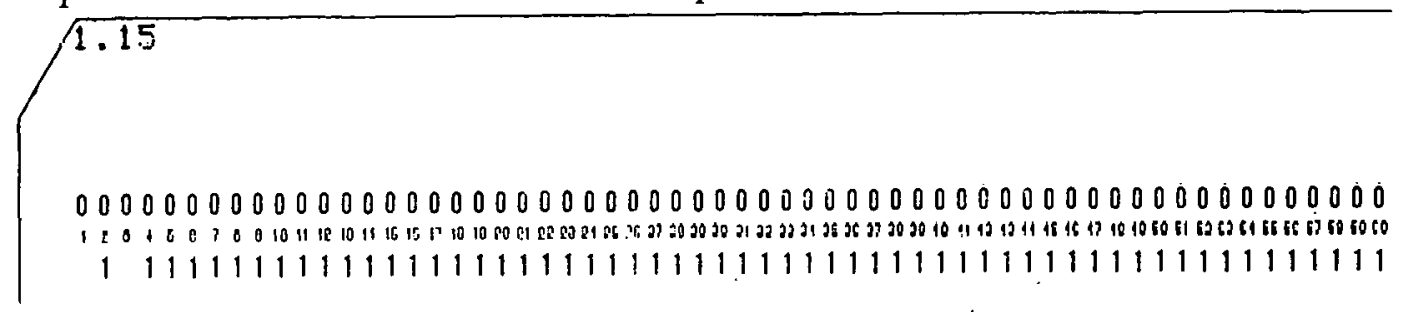

The output from the program is divided into six parts. The first part contains a description and listing of all input data and several other values related to the fin geometry. Part two contains the normal force coefficient and pitch moment coefficient of each fin and the fins summed together; if these forces and moments are not requested, this part is eliminated. Part three contains the side force coefficient and yaw moment coeficient of each fin and the fins summed together; if these force and moments are not requested, this part is eliminated. Part four contains the roll moment coefficient of each fin and the fins summed together; if this moment is not requested, this part is eliminated. Part five contains the panel normal force coefficient, hinge moment coefficient, and root bending moment coefficient of each fin; if these forces and moments are not requested, this part is eliminated. Part six contains the normal force coefficient, pitch moment coefficient, side force coefficient, yaw moment coefficient, and roll moment coefficient averaged over one $y U^{\circ}$ roll cycle; it the roll rate is specified as zero, this part is eliminated.

Parts two through five list the forces and moments as a function of roll angle for each angle of attack of the body and each roll rate. Part six lists the lorices and monitents as a function of rull rale fur each angle of attack of the body. The output in part six is used to calculated quantities such as $C_{\ell_{p}}$ and steadystate roll raise rate.

The computer run time depends on certain options chosen in the input variables. Two examples of run time on a CDC 7600 are as follows: Example 1, calculate all forces and moments ( 3 forces and 5 moments), six angles of attack of the body, and one roll rate: $39 \mathrm{~s}$. Example 2, calculate all forces and moments, six angles of attack of the body, and six roll rates: $184 \mathrm{~s}$. 
DISTRIBUTION:

0400 C. Winter

1252 G. W. Stone

4300 R. I., Peurifoy, Jr.

Attn: E. E. Ives, 4330

H. W. Schmitt, 4340

4310 C. C. Burks J. A. Hood, 4360

Attn: D. L. McCoy, 4311

5000 J. K. Galt

Attn: F. L. Vook, 5100

R. S. Claassen, 5800

5500 O. E. Jones

Attn: T. B. Lane, 5520

T. G. Priddy, 5522

5600 D. B. Shuster

Attn: A. A. Lieber, 5610

G. J. Simmons, 5640

5620 M. M. Newsom

Attn: C. E. Dalton, 5621

R. D. Andreas, 5622

5630 R. C. Maydew

5631 H. R. Vaughn (10)

5631 D. W. Barnette

5631 G. R. Eisler

5631 R. W. Greene

5631 A. E. Hodapp

5631 W. L. Oberkampf (20)

5632 C. W. Peterson

5633 S. McAlees

5634 D. D. McBride

Attn: E. L. Clark

5635 W. R. Barton

Attn: W. E. Williamson

5636 J. K. Cole

5636 H. R. Spahr

5650 D. J. Rigali

8100 W. J. Spencer

Attn: J. Barham, 8110

W. E. Alzheimer, 8120

R. D. Cozine, 8160

8150 J. L. Wirth

Attn: G. N. Beeler, 8152

8214 M. A. P'ound

3141 L. J. Erickson (5)

3151 W. L. Garner (3)

For: DOE/TIC (Unlimited Release)

DOE/TIC (25)

(J. Hernandez, 3154-4) 


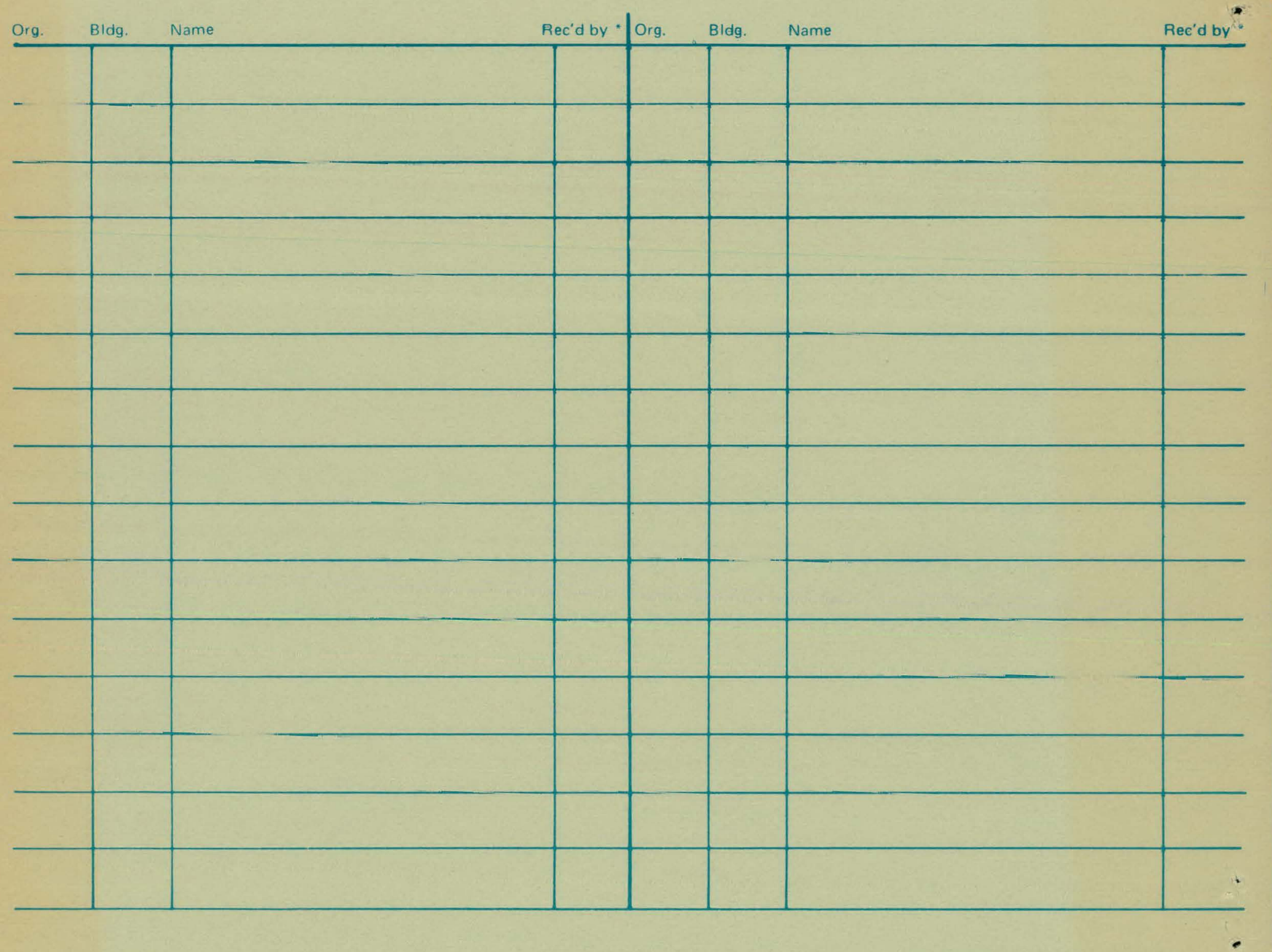

